\title{
Hemispheric asymmetries in fronto-parietal networks underlying attentional control
}

Citation for published version (APA):

Duecker, F. (2013). Hemispheric asymmetries in fronto-parietal networks underlying attentional control. [Doctoral Thesis, Maastricht University]. Maastricht University. https://doi.org/10.26481/dis.20131122fd

Document status and date:

Published: 01/01/2013

DOI:

$10.26481 /$ dis.20131122fd

Document Version:

Publisher's PDF, also known as Version of record

\section{Please check the document version of this publication:}

- A submitted manuscript is the version of the article upon submission and before peer-review. There can be important differences between the submitted version and the official published version of record.

People interested in the research are advised to contact the author for the final version of the publication, or visit the DOI to the publisher's website.

- The final author version and the galley proof are versions of the publication after peer review.

- The final published version features the final layout of the paper including the volume, issue and page numbers.

Link to publication

\footnotetext{
General rights rights.

- You may freely distribute the URL identifying the publication in the public portal. please follow below link for the End User Agreement:

www.umlib.nl/taverne-license

Take down policy

If you believe that this document breaches copyright please contact us at:

repository@maastrichtuniversity.nl

providing details and we will investigate your claim.
}

Copyright and moral rights for the publications made accessible in the public portal are retained by the authors and/or other copyright owners and it is a condition of accessing publications that users recognise and abide by the legal requirements associated with these

- Users may download and print one copy of any publication from the public portal for the purpose of private study or research.

- You may not further distribute the material or use it for any profit-making activity or commercial gain

If the publication is distributed under the terms of Article $25 \mathrm{fa}$ of the Dutch Copyright Act, indicated by the "Taverne" license above, 
Hemispheric Asymmetries in Fronto-Parietal Networks

Underlying Attentional Control

Felix Dücker 
(C) Felix Dücker, Maastricht 2013.

All rights reserved. No part of this publication may be reproduced, stored in a retrieval system or transmitted in any form or by any means, electronic, mechanical, photocopying, recording or otherwise, without prior written permission of the publisher.

The work presented in this thesis was funded by the Netherlands Organization for Scientific Research (NWO) and was conducted at Maastricht University.

Cover Joachim Richter

Production CPI Wöhrmann Print Services B.V.

ISBN 978-94-6203-472-3 


\section{Hemispheric Asymmetries in Fronto-Parietal Networks Underlying Attentional Control}

\section{Dissertation}

To obtain the degree of Doctor at Maastricht University, on the authority of Rector Magnificus, Prof. dr. L.L.G. Soete, in accordance with the decision of the Board of Deans, to be defended in public on Friday $22^{\text {nd }}$ of November 2013 at 12:00 hours by

Felix Dücker 


\section{Supervisors}

Prof. dr. A.T. Sack

Prof. dr. E. Formisano

\section{Assessment Committee}

Prof. dr. P. de Weerd (Chair)

Prof. dr. R. Goebel

Dr. P.D.C. Taylor (University of Munich, Germany)

Dr. V. van de Ven 


\section{Contents}

1 General Introduction $\quad 7$

\section{Part I}

2 Hemispheric Differences in the Voluntary Control of Spatial

Attention: Direct Evidence for a Right-Hemispheric Dominance within Frontal Cortex

3 Hemispheric Differences in the Dorsal and Ventral Fronto-Parietal Attention Network

\section{Part II}

4 The Cortex-Based Alignment Approach to TMS Coil Positioning

5 Pre-Stimulus Sham TMS Facilitates Target Detection

6 Time- and Task-Dependent Non-Neural Effects of Real and Sham TMS

7 Summary and Conclusions

Acknowledgements

Curriculum Vitae

Publications 



\section{Chapter 1}

General Introduction 
Just as all other living things, humans are capable of perceiving the outside world and respond to stimuli they encounter. Complex sensory systems have evolved over millions of years allowing us to see, hear, taste, smell, and feel the world around us. Most of the time, we are not aware of the capacity of our senses and they work so seemingly simple and unobtrusively that we take perception for granted. The truth is, however, that perception is a complex process and it is very challenging to effectively deal with the abundance of incoming sensory information. There are countless everyday life situations where the limits of perception become noticeable even though our sensory organs are working just fine.

When reading a book, for example, we cannot simply take a look at the entire page and extract all the sentences at once. Instead, we move our eyes in an automatic way successively from one word to the next completing each sentence step by step. To some extent, this reading technique is a consequence of the poor resolution of peripheral vision. When looking at this asterisk * with fixed eyes, it is almost impossible to identify surrounding words that are more than a few centimeters away. This limitation forces us to continuously move our eyes to bring, e.g., words towards the center of the visual field where the resolution is much better. In fact, we make so called saccadic eye movements all the time, up to four times per second, in order to gather as much information as possible but we inevitably miss out on many details that are nevertheless present. Staying with the previous example, being immersed in a fascinating book often makes people completely oblivious of their surroundings. They might sit in a train, turning page after page, without ever noticing what nearby people are talking about, how the sun goes down, or that they missed their stop. Clearly, not all the information that is picked up by our senses is actually perceived. It seems that we have limited resources and we can only commit them to a small portion of incoming sensory information at once. On the upside, this is not a random process and we can generally align this selection process with our goals and interests. For example, when searching for a particular book in a bookshelf, it really helps to know the color of the book cover. This knowledge can act as a filter that allows scanning along the books rather quickly without wasting time on those books that have the wrong color anyway. 
What all these examples have in common is the concept of attention, a mechanism that allows selection of those aspects of incoming sensory information that are most relevant to us. Attention has been famously defined by William James in 1890, stating that: "Everyone knows what attention is. It is the taking possession by the mind, in clear and vivid form, of one out of what seem several simultaneously possible objects or trains of thought. Focalization, concentration, of consciousness are of its essence. It implies withdrawal from some things in order to deal effectively with others, and is a condition which has a real opposite in the confused, dazed, scatterbrained state which in French is called distraction, and Zerstreutheit in German.”. This definition describes attention as a very general and fundamental process that plays an important role across various mental domains. For that reason, attention has become one of the most popular and active areas of research in neuroscience. Maybe unsurprisingly, the field of attention research fanned out tremendously over the years and attention is no longer seen as one unitary process. Instead, it is nowadays common to distinguish between different kinds of attention, at least conceptually. In order to set the stage for this thesis, a few core concepts and theories of attention will be introduced in the next sections, covering five lines of research. First, a neuropsychological syndrome that results from brain damage, called spatial neglect, will be described that has inspired two competing theories on attention. Second, one particular experimental paradigm (Posner task) that is commonly used in healthy humans to investigate attention will be presented. Third, a functional-anatomical model of brain areas involved in attention based on neuroimaging studies will be discussed. Fourth, the behavioral consequences of disrupting brain areas underlying attention with transcranial magnetic stimulation in healthy humans will be summarized. Fifth, the effects of attention on the neural representations of sensory stimuli will be briefly introduced. Taken together, these lines of research directly influenced the work presented here.

Finally, answering content-driven research questions always goes hand in hand with methodological considerations and sometimes leads to improvements of existing methodology and/or identification of previously unaddressed problems. The research on attention presented here inspired a series of experiments that relate to very general issues in the context of transcranial magnetic stimulation, a method that 
is widely used in cognitive neuroscience. Background information for this methodological work will be given at the end of this introduction.

\section{Cognitive Neuropsychology}

The idea that the brain is where the mind is located dates back thousands of years (Bear, Connors, \& Paradiso, 2001). Already in ancient Greece, Hippocrates (460 BC - $370 \mathrm{BC}$ ) expressed that "Men ought to know that from nothing else but the brain come joys, delights, laughter and sports, and sorrows, griefs, despondency, and lamentations.". Back then, this view was very controversial and famous thinkers as Aristotle had completely opposing views arguing that the heart was the seat of intelligence, with the brain merely being responsible for cooling the blood. But it took until the mid- $19^{\text {th }}$ century, when it was finally shown experimentally that different parts of the brain underlie different mental functions (Pearce, 2009). Jean Pierre Flourens was the first to systematically damage distinct parts of the brain in animals and to observe the functional consequences. Among other things, he demonstrated that removal of the cerebral hemispheres caused complete loss of perception, cognition, and motor control whereas removal of the cerebellum affected mainly motor coordination. Due to technical limitations, Flourens was unable to explore potential brain-behavior relationships in more detail, but a first step in understanding how the brain works was made. The next seminal discovery was made by Paul Broca in 1861 who investigated the consequences of brain damage in humans. He discovered that a lesion at the inferior frontal gyrus caused deficits in speech production but left speech comprehension intact. Until today, this part of the brain is called Broca's area indicating the importance of his work. Other brain areas with other functions were soon discovered and the concept of functional specialization and localization became a cornerstone of cognitive neuroscience which is highly relevant until the present day (Rorden \& Karnath, 2004).

In Broca's time, the brain could only be investigated post mortem, that is, after a person had died which obviously posed many practical problems. In the $20^{\text {th }}$ century, however, it became possible to acquire anatomical images of the brain in vivo 
allowing examination of the consequences of brain damage on a larger scale. The field of cognitive neuropsychology emerged with the primary goal to understand the functional role of brain areas based on patients with brain damage. The general rationale in so called lesion studies is to identify which brain areas are damaged in any given patient and to carefully assess the mental deficits that go along with it. Those functions that are found to be impaired are then believed to be a function of the brain area in question. This approach has provided valuable insights into the functional organization of the brain even though there are various limitations and problems that will be discussed later.

Among the most common consequences of brain damage is a neuropsychological syndrome called spatial neglect that can be observed after lesions to frontal, parietal, or sub-cortical structures (Corbetta, Kincade, Lewis, Snyder, \& Sapir, 2005). Spatial neglect is characterized by impaired attentional processing within the space contralateral to the lesion's location. As a consequence, patients typically fail to attend, explore, and act upon one side of space which also leads to peculiar effects in the perceptual domain. For example, when a patient with right hemisphere damage is required to copy a drawing he/she will typically ignore the left side entirely and only work on those parts of the drawing that are on the right side (Figure 1). Similarly, patients with spatial neglect might only eat the food on one half of their plate or fail to put both arms into their sleeves. Interestingly, the phenomenon of spatial neglect is more common and severe after right hemisphere damage than after left hemisphere damage, suggesting a functional asymmetry of the mechanisms underlying spatial attention (Mesulam, 1981). Based on this
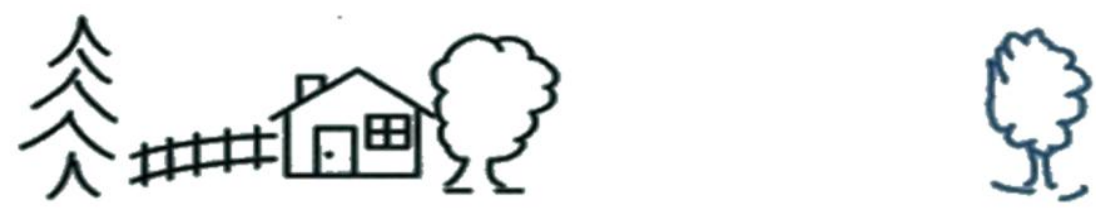

Figure 1. A patient with spatial neglect will typically fail to copy those elements of a drawing that are contralateral to the lesion. In this case, only the tree on the right side of the original drawing is attended to and can be perceived while the left side is completely ignored. 
observation, two competing theories regarding the neural dynamics underlying spatial attention have emerged, namely Heilman's "hemispatial" theory (Heilman \& Abell, 1980) and Kinsbourne's “opponent processor” model (Kinsbourne, 1977), both accounting for this asymmetry but proposing very different mechanisms.

According to Heilman's "hemispatial" theory (Figure 2a), the right hemisphere is dominant in spatial attention because it mediates attention shifts to both hemifields whereas the left hemisphere only mediates attention shifts to the right hemifield. In case of left hemisphere damage (Figure 2b), only mild attentional deficits are expected because the right hemisphere can still shift attention to both hemifields thereby compensating for the damaged left hemisphere. Consequently, the ability to shift attention to both hemifields remains largely intact. In case of right hemisphere damage (Figure 2c), however, such overlap in function does not exist and functional compensation is impossible because the intact left hemisphere only mediates attention shifts to the right hemifield. Consequently, the ability to shift attention to the left hemifield is lost resulting in spatial neglect of the left hemifield.

According to Kinsbourne's “opponent processor" model (Figure 3a), each hemisphere has a natural attention bias to the contralateral hemifield with the

a

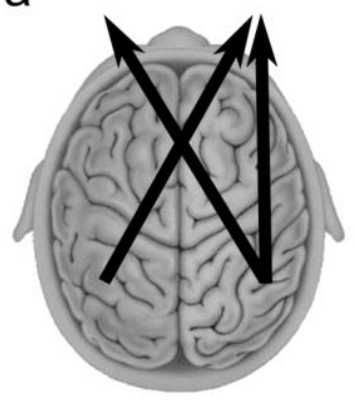

b

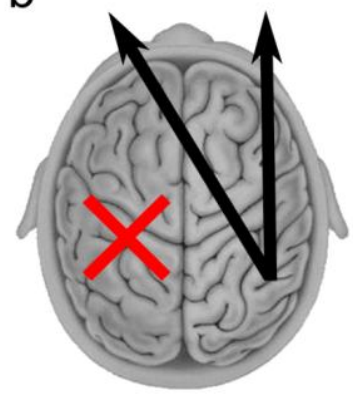

C

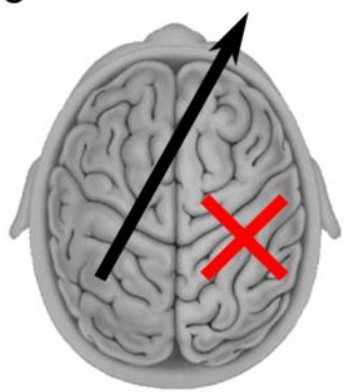

Figure 2. Heilman's "hemispatial" theory. a) The functional role of each hemisphere under normal conditions, that is, the right hemisphere mediates attention shifts to both hemifields whereas the left hemisphere mediates attention shifts to the contralateral hemifield. b) The consequences of left hemisphere damage. Only the function of the right hemisphere is preserved but attention can still be shifted to both hemifield due to overlap in function. c) The consequences of right hemisphere damage. Only the function of the left hemisphere is preserved so that attention can no longer be shifted to the left hemifield, resulting in neglect of the left hemifield. 
rightward bias of the left hemisphere being stronger than the leftward bias of the right hemisphere. Under normal conditions, the two hemispheres are kept in balance due to inter-hemispheric competition but damage to either side leaves the contralesional intact hemisphere unopposed. As a result of this reduced inhibition, the contralesional hemisphere becomes over-activated and causes an ipsilesional attention bias. In case of left hemisphere damage (Figure 3b), only mild attentional deficits are expected because the leftward bias of the right hemisphere is relatively small. Consequently, the ability to shift attention to both hemifields remains largely intact. In case of right hemisphere damage (Figure 3c), however, the strong rightward bias of the left hemisphere is uncovered and attention is strongly biased to the right hemifield. Consequently, the ability to shift attention to the left hemifield is suppressed resulting in spatial neglect of the left hemifield. Taken together, the explanatory approaches of both theories are very different but the predicted behavioral consequences of left and right hemisphere damage are very similar making it difficult to dissociate them empirically. Even though the debate between both theories is far from being settled, Heilman's "hemispatial" theory has long been more popular and well-known and right-hemispheric dominance in spatial attention is widely assumed.

a

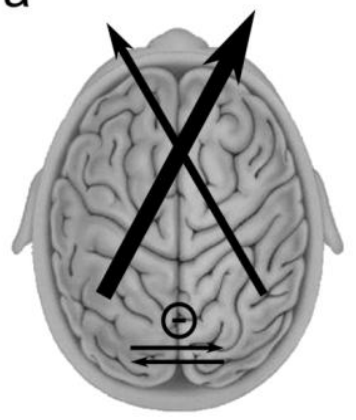

b

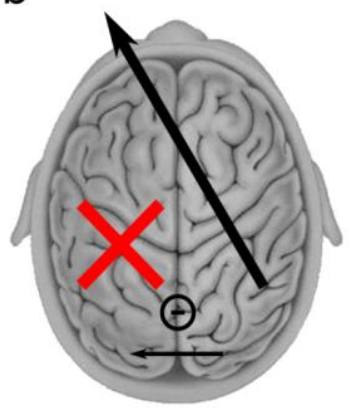

C

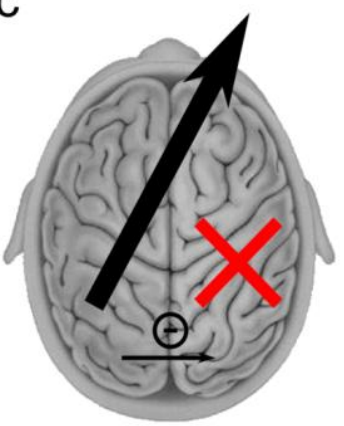

Figure 3. Kinsbourne's "opponent processor" model. a) The functional role of each hemisphere under normal conditions, that is, each hemisphere has an attentional bias towards the contralateral hemifield and there is a balance due to inter-hemispheric inhibition. b) The consequences of left hemisphere damage. The rather small bias of the right hemisphere is unopposed leading to a small attention bias to the left. c) The consequences of right hemisphere damage. The strong bias of the left hemisphere is unopposed leading to a strong attention bias to the left, effectively resulting in neglect of the left hemifield. Please note that the thickness of the arrows indicates the strength of the bias. 
As pointed out before, there are some limitations of the lesion approach that should not go unmentioned (for an overview, see Rorden \& Karnath, 2004). To begin with, the lesion's location and extent is not experimentally controlled under normal circumstances. Brain lesions are usually the result of stroke which can happen throughout the brain and the damage of neural tissue depends on the specific effects the stroke has on blood supply. As a consequence, some brain areas are more prone to be affected than others and, more importantly, this makes it very difficult to find patient groups with consistent brain damage. In most cases, damage will not be limited to the brain area of interest so that adjacent areas are also dysfunctional. Similarly, swellings around the lesion often have an effect on neural activity as well and this might vary during the time course of healing, complicating things even further. Finally, the functional organization of the brain can actually change after brain damage which can greatly complicate the interpretation of lesion studies. Taken together, the anatomical definition of a brain lesion and its functional consequences are not as straightforward as one might assume and to infer the function of a brain area with such variable and coarse data is very difficult despite the conceptual simplicity of this approach. With these limitations of the lesion approach in mind, the general claim of a functional asymmetry between the left hemisphere and right hemisphere remains in place. In order to directly test Heilman's "hemispatial” theory against Kinsbourne's "opponent processor" model, however, more powerful techniques are required. Ideally, one would like to investigate attentional processes and their underlying brain mechanisms in healthy humans. Fortunately, such methods are nowadays available but before exploring them in detail, a closer look at how attentional processes are commonly measured on the behavioral level will follow.

\section{Psychophysics}

In most general terms, there are two different kinds of attention, namely overt and covert attention. In case of overt attention, the senses are directed to a specific stimulus in order to enhance processing. This is what people normally do and it is 
readily observable in everyday life. For example, we move the eyes from one word to next when reading a book or turn the head when carefully listening to a faint sound. In case of covert attention, however, there is only an internal mechanism that selects a specific stimulus while any kind of orienting behavior is lacking. There is a long-standing debate concerning the relationship of overt and covert attention but this is beyond the scope of this introduction (but see Moore, Armstrong, \& Fallah, 2003). Most importantly, covert attention is particularly interesting because it allows investigating attentional selection in a very isolated state. As long as the eyes do not move, the incoming sensory information is essentially constant so that observed effects can be attributed to attention instead of other confounding factors.

Next to the overt/covert dichotomy, there is another important division of attentional processes, namely endogenous and exogenous control of attention (Corbetta \& Shulman, 2002). Shifts of spatial attention are endogenous when they are initiated in a voluntary fashion based on current goals or expectations. For example, when providing information about the color of a book cover, finding the book in questions is typically much easier. Shifts of spatial attention are exogenous when they are triggered by salient or unexpected stimuli making it difficult to suppress them. For example, no matter how interesting a book may be, a loud sudden noise will be very effective in interrupting a reader who may then look around to find out what is going on before turning back to the book. The combination of both types of attentional control is imperative for goal-directed behavior while remaining sensitive to unexpected but potentially relevant events.

One of the most famous experimental paradigms in cognitive neuroscience is a specific class of spatial cueing tasks, invented by Michael Posner in the 1980s and designed to investigate covert spatial attention (Posner, Snyder, \& Davdison, 1980; Posner, 1980). The principle idea is that the allocation of attentional resources to a particular spatial location selectively facilitates the processing of incoming sensory information at that location so that a behavioral response contingent on a stimulus is faster or more accurate. Roughly speaking, there are two versions of the so called Posner task either requiring endogenous or exogenous shifts of spatial attention. Typically, participants look at a fixation cross at the center of the screen and a cue is 
briefly presented. The cue is followed by a target stimulus in the periphery either on the left or right side of the fixation cross. Participants have to indicate the detection of the stimulus with a button press irrespective of target location. Crucially, only the type of cue determines whether endogenous or exogenous attention shifts are elicited.

Figure 4a shows a spatial cueing task that requires endogenous shifts of spatial attention. Central symbolic cues are used that provide information about where the target stimulus is most likely to appear. In this case, arrow heads either point to the left side, right side, or in both directions but color changes of the fixation cross could serve the same purpose. Participants are asked to interpret the cue and to shift their attention towards the corresponding side of space without moving their eyes. After a short time interval, the target stimulus appears and participants respond as fast as possible with a button press. Those trials with arrow heads pointing in both directions serve as baseline condition where no information about the location of the upcoming target stimulus is provided. The target can either appear on the left side or the right side with equal probability and, hence, there is no reason to shift attention prior to target appearance (neutral trials). Those trials with directional cues, however, have high predictive value, meaning that they correctly tell where the target stimulus is going to appear most of the time (around $80 \%$ correct).

\section{a ENDOGENOUS}
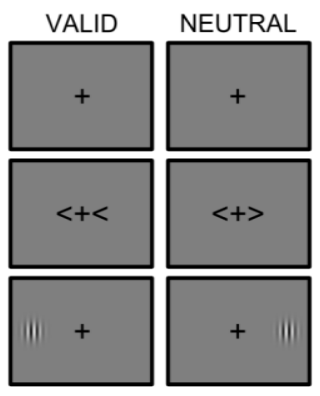
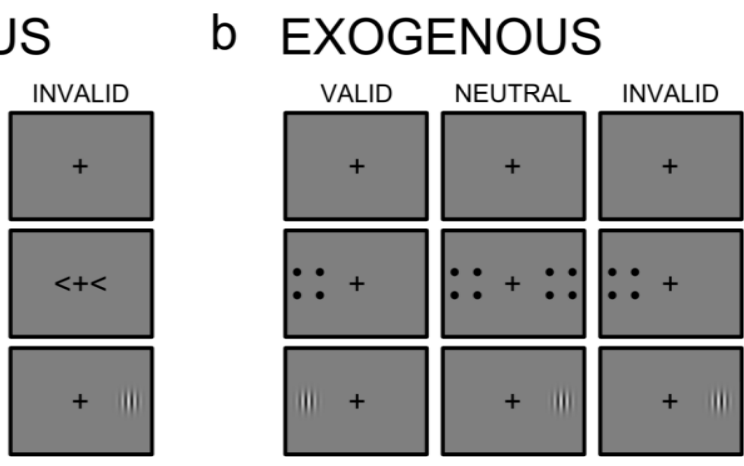

Figure 4. Typical spatial cueing tasks based on the work of Michael Posner. a) An example of endogenous shifts of attention where central symbolic cues are used to provide information about the probably location of the upcoming target stimulus. Trials are valid, neutral, or invalid, depending on the cue-target combination. b) An example of exogenous shifts of attention where peripheral cues are used to automatically pull spatial attention to one side of space. Trials are valid, neutral, or invalid, depending on the cue-target combination. 
As a consequence, participants shift their attention towards the cued location, trusting that the cue indeed provides correct information. On so called valid trials, the target stimulus is actually presented at the cued location but, occasionally, the cue-induced spatial expectancy is violated and the target stimulus appears at the opposite location (invalid trials). Obviously, the cue effectiveness heavily relies on its predictive value and participants will make use of the cues as longs as it is beneficial most of the time. As one might expect, performance is not identical across valid, neutral, and invalid trials but rather shows a specific pattern. As pointed out before, neutral trials serve as a baseline condition that includes perceptual processing, target detection, and the behavioral response but lacks attentional components based on spatial expectancies. In case of valid trials, reaction times are typically faster compared to neutral trials (attentional benefits) indicating more efficient processing due to successful voluntary attention shifts. In case of invalid trials, reaction times are typically slower compared to neutral trials (attentional costs) indicating that incorrect information about the target location requires reorienting of attention to the unexpected target location after target appearance.

Figure $4 \mathrm{~b}$ shows a spatial cueing task that elicits exogenous shifts of spatial attention. In this case, peripheral cues are used (e.g. small dots) that briefly light up either on the left side, right side, or on both sides. Just as in the previous task, the target stimulus is presented shortly after the cue and participants are required to respond with a button press as fast as possible. Importantly, the cues are completely task-irrelevant and do not provide any information about where the target stimulus is going to appear. Instead, the cue causes an automatic shift of spatial attention towards the cued location. Those trials with bilateral cues serve as a baseline condition whereas the remaining trials are either valid or invalid depending on whether or not the cue and target stimulus were presented on the same side. Analogous to the previous version of the Posner task, reaction time data can then be analyzed in terms of attentional benefits and attentional costs which are again interpreted as reflecting attentional orienting and reorienting, respectively.

Even though both tasks are very similar, it is important to stress their differences. As has been mentioned before, endogenous cues have to be predictive in order to be effective. Participants will not necessarily make use of the cue when it is not 
behaviorally advantageous. In contrast, exogenous cueing even works with nonpredictive cues supporting the idea that they trigger an automatic process. Moreover, the time interval between cue and target stimulus is a critical variable in both tasks. While endogenous cueing produces the strongest effect when the target stimulus appears around $500 \mathrm{~ms}$ after cue onset, exogenous cueing is most effective for much shorter cue-target intervals (around $100 \mathrm{~ms}$ ). Again, this supports the notion that an endogenous shift of spatial attention is a voluntary act that is contingent on the interpretation of a central symbolic cue. Naturally, such a process requires some time to be executed whereas an automatic shift of spatial attention triggered by an exogenous cue happens automatically and therefore much faster.

Finally, a word of caution, the distinction between endogenous and exogenous components within each task is not as clear-cut as it might appear from the description above. While valid and neutral trials still nicely fit into the picture, invalid trials during an endogenous cueing tasks actually have a lot in common with exogenous cueing because the target stimulus appears unexpectedly in the periphery. For that reason, the conceptual differences introduced here should not be overstretched and a closer look at Posner tasks would reveal more sub-processes than are discussed here. Nevertheless, each version of the Posner task isolates specific attentional processes in a well-controlled way and allows measuring these processes in terms of reaction time differences between neutral and valid/invalid trials. As will be shown in the next section, both versions of the Posner task have been widely used in combination with neuroimaging techniques to identify the brain areas underlying spatial attention control.

\section{Functional Magnetic Resonance Imaging}

The most important development in cognitive neuroscience possibly is the advent of functional magnetic resonance imaging (fMRI) which allows measuring brain activity in humans, albeit indirectly. The basic principle behind fMRI is that neuronal activity is accompanied by local changes of blood oxygenation. Due to the different magnetic properties of oxygen-rich and oxygen-poor blood, these changes 
can be picked up by an fMRI scanner resulting in the so called blood oxygenation level dependent (BOLD) response. Importantly, the BOLD response can be obtained for the entire brain within a couple of seconds with high spatial resolution (typically $3 \times 3 \times 3 \mathrm{~mm}$ ). As a result, it is possible to investigate the brain activity changes while participants perform any kind of task in the scanner. Of course, this has also been done in the context of attention and many years of research have resulted in a consistent set of brain areas that are involved in endogenous and exogenous attention.

Based on neuroimaging studies, Corbetta and Shulman have proposed a functional-anatomical model of attentional control (Figure 5) that revolves around two distinct but interacting networks comprising frontal and parietal brain areas (Corbetta \& Shulman, 2002, 2011). On the one hand, there is a bilateral dorsal fronto-parietal network including the frontal eye field (FEF) and intraparietal sulcus (IPS). This network is directly related to shifts of spatial attention and modulates sensory areas in a top-down way. On the other hand, there is a right-lateralized ventral fronto-parietal network including the temporo-parietal junction (TPJ) and ventral frontal cortex (VFC). This network acts as a circuit breaker of the

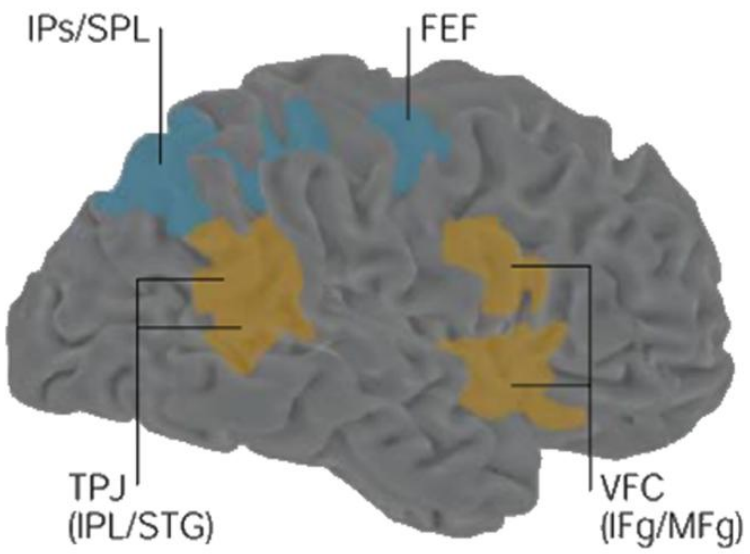

Figure 5. Functional-anatomical model as proposed by Corbetta and Shulman in 2002 (image taken from this source). The dorsal fronto-parietal network is shown in blue. The ventral fronto-parietal network is shown in yellow. 
dorsal network in case an unexpected or salient stimulus occurs that requires reorienting of attention. Given the functional role of the dorsal network, one would expect that neuroimaging studies reveal some degree of functional specialization within nodes of the dorsal network that match either Heilman's "hemispatial" theory or Kinsbourne's “opponent processor” model. Surprisingly, neuroimaging studies have thus far have failed to provide consistent evidence in favor of either view. The majority of studies observed bilateral activation in IPS and FEF; neither supporting the notion of a contralateral bias nor of right-hemispheric dominance (Hopfinger, Buonocore, \& Mangun, 2000; Kastner, Pinsk, De Weerd, Desimone, \& Ungerleider, 1999; Kincade, Abrams, Astafiev, Shulman, \& Corbetta, 2005; Shulman et al., 2010). As in most cases, there are some exceptions but they do not converge towards one single conclusion either. For example, the "opponent processor" model is supported by the presence of topographic representations in the dorsal network that only span the contralateral hemifield in each hemisphere (Silver \& Kastner, 2009). Similarly, activation is sometimes higher in a hemisphere when shifting attention towards the contralateral hemifield (Szczepanski, Konen, \& Kastner, 2010) but evidence in favor of the "hemispatial" theory has recently been reported as well (Sheremata, Bettencourt, \& Somers, 2010). Either way, with two competing theories that propose fundamentally different mechanisms of spatial attention control, the evidence base provided by neuroimaging studies is somewhat disappointing, opening the doors for alternative explanations why spatial neglect is more common after right hemisphere damage.

Recently, Corbetta and Shulman have argued that spatial neglect is primarily related to lesions of the ventral fronto-parietal network (Corbetta \& Shulman, 2011). Even though the localization of lesions leading to spatial neglect is rather heterogeneous, there are some indications that brain damage to ventral areas are indeed very prominent in neglect patients (Corbetta et al., 2005; Corbetta \& Shulman, 2011). Most importantly, the ventral fronto-parietal network is strongly lateralized to the right hemisphere offering an explanation for the prevalence of spatial neglect after right hemisphere damage. The principal idea is that damage to the ventral network also indirectly affects the dorsal network due to dysfunctional interactions between both networks. Because the ventral network is right-lateralized, 
right hemisphere damage will have a stronger (indirect) effect on the dorsal network than left hemisphere damage. On a positive note, this explanation offers a coherent framework of spatial attention control and seems to fit well with lesion studies in neglect patients. However, the proposed dynamics of dorsal and ventral interaction still lack empirical support and the precise workings of the dorsal fronto-parietal network remain largely unspecified. Heilman's "hemispatial" theory and Kinsbourne's "opponent processor" model cannot be discarded based on current neuroimaging studies and, interestingly, transcranial magnetic stimulation has provided a different perspective on the discussion at hand.

\section{Transcranial Magnetic Stimulation}

Transcranial magnetic stimulation (TMS) is a non-invasive brain interference technique that allows manipulation of brain activity in humans by exposing the brain to a rapidly changing magnetic field (Hallett, 2000; Pascual-Leone, Walsh, \& Rothwell, 2000; Sack, 2006; Walsh \& Cowey, 2000). TMS is applied by positioning a TMS coil on the head and whenever a TMS pulse is given, the cortical area under the TMS coil will be stimulated. TMS coils are purpose-built to generate very focal magnetic fields so that only a small patch of the cortical surface is affected by TMS. This allows stimulation of individual functional brain areas with only minor interference of neighboring areas. As a consequence, TMS is one of the very few techniques that can be used in humans to investigate how directly induced brain activity changes influence task performance. The rationale in the majority of TMS experiments is that if TMS over a particular brain area has an effect on a specific function then this brain area is causally involved in this process. In this sense, TMS is capable of revealing causal structure-function relationships which gives TMS an almost unique position in cognitive neuroscience. Other methods, such as fMRI, are often limited to observing task-correlated brain activity changes that do not necessarily imply causality. Interestingly, there is a strong conceptual link between TMS in healthy humans and lesion studies in patients with brain damage. Just as lesions cause functional deficits, TMS can be used to temporarily disrupt a brain 
area in order to observe the functional consequences. Due to this similarity, TMS is commonly called the "virtual lesion" approach even though the disruptive effects of TMS are far less severe than those of brain damage. In fact, most TMS effects are only observable as subtle changes of reaction times or accuracy and do not lead to impairments in a practical sense. Importantly, many of the problems associated with lesion studies do not apply to TMS and the benefit of investigating the functional role of brain areas in the healthy human brain cannot be underestimated. However, TMS is not without problems either and some of them will be discussed in a later section.

Returning to spatial attention control, several TMS experiments have been conducted to investigate the behavioral consequences of disrupting the core nodes of the dorsal fronto-parietal network. Interestingly, these studies have produced novel evidence concerning the functional role of these brain areas that neuroimaging studies have failed to provide. To begin with, TMS over parietal brain areas within the dorsal network have consistently provided evidence in favor of Kinsbourne's “opponent processor” model (Dambeck et al., 2006; Hilgetag, Theoret, \& PascualLeone, 2001; Seyal, Ro, \& Rafal, 1995; Silvanto, Muggleton, Lavie, \& Walsh, 2009). For example, Hilgetag et al. (2001) found contralateral impairments and ipsilateral enhancements of target detection after TMS over left and right IPS conforming to Kinsbourne's notion of a contralateral bias and inter-hemispheric competition. In accordance with this interpretation, Dambeck et al. (2006) revealed that target detection is unaffected when TMS is applied simultaneously over left and right parietal cortex whereas unilateral TMS impairs detection of contralateral stimuli. In contrast, the functional asymmetry of frontal areas within the dorsal network has rarely been addressed and those studies that directly compared the functional role of right and left FEF were unable to dissociate attentional from perceptual effects (Grosbras \& Paus, 2002, 2003; Silvanto, Lavie, \& Walsh, 2006). With this limitation in mind, TMS over frontal areas has produced results that are in favor of Heilman's "hemispatial" theory, contrasting the findings in parietal cortex discussed above. For example, it has been shown that right FEF stimulation increases perceptual sensitivity in both hemifields whereas left FEF stimulation effects are limited to the contralateral hemifield (Grosbras \& Paus, 2003). The same 
pattern of results has been observed in terms of changed phosphene thresholds when assessing the top-down influence of FEF on cortical excitability in extrastriate cortex (Silvanto et al., 2006).

Taken together, these TMS experiments generally support the role of the dorsal network in attentional control, as already indicated by neuroimaging studies. Moreover, it appears that the bilateral activation of IPS and FEF that is commonly found with fMRI does not fully reflect the organizational principles of this network and even is to some extent misleading. There seems to be a more detailed functional differentiation and both Heilman's "hemispatial" theory and Kinsbourne's “opponent processor" model have received supportive evidence. Obviously, the discussion is far from being settled and there has not been a large-scale effort to investigate the precise attentional deficits that follow TMS over nodes of the dorsal and ventral network with the same TMS parameters, tasks, and outcome measures. Only then, a direct comparison of the functional properties of each node in these networks can be obtained. This thesis represents an effort to achieve this goal and provides novel evidence that has direct relevance for current functional-anatomical models of spatial attention control.

\section{Top-Down Attentional Modulation of Sensory Areas}

Previous sections have introduced attention as a cognitive process and the underlying mechanisms have been described at the system level. The primary focus was on the brain areas involved in attentional control and their functions. The work presented in this thesis is also situated on this level and the methods being used are ideally suited for that. However, this leaves out an important aspect of attention research, namely the effects of attention on perception and the underlying neurophysiological mechanisms. How does attention affect processing of incoming sensory information in visual cortex? Addressing this question requires a level of investigation that is largely inaccessible with the methods used here. There are, however, several general principles involved in attentional modulation of incoming sensory information that may play a central role in producing the observed 
behavioral effects. While a thorough discussion of these principles is beyond the scope of this thesis, they will be briefly described below for completeness of background information.

Attention has been shown to modulate neuronal activity in sensory areas (Kastner \& Ungerleider, 2000; Reynolds \& Chelazzi, 2004). In those cases where only a single stimulus is presented, attention selectively increases the response of those neurons that are tuned to the properties of that stimulus; consistent with a gain modulation view (McAdams \& Maunsell, 1999; Reynolds, Pasternak, \& Desimone, 2000). For example, a stimulus with low physical contrast is processed as if it had higher contrast effectively increasing the signal-to-noise ratio. An attended stimulus is therefore more readily processed and perceptually enhanced (Carrasco, Ling, \& Read, 2004). In those cases where two stimuli are presented, a situation of competition arises in which attention can bias the neuronal response in favor of the attended stimulus (Reynolds, Chelazzi, \& Desimone, 1999). Importantly, this does not necessarily imply neuronal response increases because this so called biased competition framework also allows attention to give a weaker stimulus an advantage over a stronger stimulus resulting in neuronal response decreases. Recently, the above mentioned principles have been integrated into the "normalization model" of attention which explains different manifestations of attentional modulation within a single computational framework (Reynolds \& Heeger, 2009). Finally, a full explanation of the effects of attention on perceptual processes in sensory areas also requires a mechanism that actively enhances or suppresses neuronal activity. The most prominent view is that oscillations play a key role in controlling the dynamics of sensory processing. Oscillations in the gamma range have been associated with attentional selection whereas oscillations in the alpha range have been associated with inhibitory processes (Engel, Fries, \& Singer, 2001; Jensen \& Mazaheri, 2010). For the present purpose, the critical point is that any TMS effect on attentional processes (as assessed on the behavioral level) most likely is accompanied by altered top-down modulation of sensory areas with effects also occurring on the neurophysiological level. The work presented in this thesis focuses on the functional properties of fronto-parietal brain areas that exert a top-down influence on sensory 
areas without investigating the consequences of these top-down modulations on sensory cortices per se.

\section{Methodological Issues in TMS research}

Previous sections have shown that there are well-defined brain areas constituting distinct fronto-parietal networks underlying attentional control. This offers huge potential to reveal their individual functional roles by a precisely localized TMS interference. Indeed, one of the major advantages of TMS over other non-invasive brain interference techniques is its relatively good spatial resolution. To illustrate, when moving a typical figure-of-eight TMS coil along the hand area of primary motor cortex, it is possible to evoke muscle twitches in each finger separately even though the underlying representations are only a few millimeters apart. This precision allows very selective targeting of functional brain areas and is a prerequisite for drawing strong conclusions regarding structure-function relationships. This strength, however, also poses a practical challenge, namely how to accurately position the TMS coil on the head, so that the brain area of interest is actually affected by TMS instead of another brain area in the vicinity? One aspect of this question can be solved with a neuronavigation system. Once anatomical data of a participant are available, it is possible to bring representations of the head, brain, and TMS coil in a common reference space so that their relative positions can be visualized in real-time. However, this is not sufficient for optimal TMS coil positioning because brain anatomy alone does not necessarily predict where a functional brain area is located. Therefore, it is ideal to also obtain individual fMRI data for each participant (Sack et al., 2009). For many reasons, however, this is not always done and a common alternative approach is to use already existing fMRI group data that are reported as a set Talairach coordinates in the literature. These coordinates are then used to estimate where a functional brain area is located in any given participant while only requiring anatomical data. Generally speaking, TMS coil positioning based on anatomical data (with or without Talairach coordinates) is suboptimal because the obtained TMS target points will not match the exact location 
of a functional brain area in most cases but they are nevertheless widely used for practical, experimental, or economic reasons. Given that the quality of TMS coil positioning can have a major influence on the success or failure of a TMS experiment, it is very important to get the highest precision possible with data that are available. A major limitation of TMS coil positioning based on Talairach coordinates is that the underlying anatomical alignment completely neglects the macro-anatomical organization of the brain so that this source of anatomical variability across participants is not taken into account. As a consequence, the extent of functional-anatomical correspondence remains unexploited while this information could actually be used to improve TMS coil positioning.

Another important aspect of any TMS experiment is the inclusion of control conditions. Next to the intended neural effects of TMS, every TMS pulse produces a distinct clicking sound and sensation on the head. This creates a strong need for appropriate control conditions because it is vital to ensure that effects of interest are not the result of these non-neural side effects of TMS but are caused by the TMSinduced brain activity changes. Particularly in attention research, these non-neural effects can be problematic because the clicking sound of the TMS coil is a salient sensory event that essentially serves as an exogenous cue. As such, it might pull spatial attention away from the current locus of attention, act as a warning signal, or simply distract participants resulting in less focused attention. Many different control strategies have been developed to deal with these issues and they are all based on the concept of specificity. The general idea is that a TMS effect is thought to be 'neural' when it is specific to a stimulation site, time point of stimulation, task, or other parameters. The implicit assumption is that the non-neural effects lack such specificity and therefore do not produce differences between conditions. We have conducted two experiments that challenge this assumption on empirical grounds and provide an empirical foundation for control strategies in TMS research. 


\section{Outline of Thesis}

The work described in this thesis is subdivided into two parts. All experiments revolve around transcranial magnetic stimulation (TMS), a widely-used brain interference technique in cognitive neuroscience. The experiments presented in Part I aim to answer specific content-driven research questions in the context of spatial attention control whereas Part II is about methodological aspects of TMS that are of particular importance in attention research but also have a bearing on TMS methodologies in general.

The experiments presented in Part I investigate three explanatory approaches for the predominance of spatial neglect after damage to the right hemisphere. On the one hand, Heilman's "hemispatial" theory and Kinsbourne's "opponent processor" model propose functional asymmetries between the two hemispheres in spatial attention control (Heilman \& Abell, 1980; Kinsbourne, 1977). Even though these theories predate the advent of neuroimaging, these functional asymmetries can be expected to be localized in the dorsal fronto-parietal network. On the other hand, Corbetta and Shulman argue that spatial neglect originates in the right-lateralized ventral fronto-parietal network and spatial attention control is only indirectly affected due to disrupted interactions between the ventral and dorsal system (2011). Based on these theories, we formulated specific hypotheses that can be tested with TMS. Using a "virtual lesion" approach, we applied TMS over core nodes of the dorsal and ventral fronto-parietal network in order to assess the functional consequences of FEF, IPS, and TPJ disruption. Given the proposed importance of hemispheric asymmetries in spatial attention control, we compared the effects of TMS for each brain area in both hemispheres. The effects of TMS were assessed on the behavioral level with a well-controlled psychophysical task that allowed separating distinct attentional processes such as orienting of attention and reorienting of attention. These experiments were specifically designed to provide novel evidence regarding the mechanisms underlying spatial attention control and to inform current functional-anatomical models. In Chapter 2, the main focus was to test Heilman's "hemispatial" theory against Kinsbourne's "opponent processor" 
model. TMS was applied to the frontal part of the dorsal network, namely over left and right FEF, based on individual fMRI data to precisely localize the activation hotspots in each participant. In Chapter 3, we set out to extend our previous findings and applied TMS over the parietal part of the dorsal network, namely over left and right IPS. Moreover, we also included the ventral network, namely left and right TPJ, in order to examine the effects of ventral network disruption on functions of the dorsal network. In contrast to the first experiment, TMS target points were defined based on Talairach coordinates from the literature which, in hindsight, severely compromised TMS localization accuracy and, as a consequence, the entire experiment. However, this setback has inspired the work presented in Chapter 4.

The experiments presented in Part II address methodological aspects of TMS research. In Chapter 4, we present a novel approach for TMS coil positioning based on individual anatomical data that outperforms alternative approaches such as targeting based on Talairach coordinates. These improvements are achieved by exploiting curvature information of the cortical surface for alignment to a group average brain that contains functional group data. In this way, we account for the macro-anatomical variability across participants and take advantage of the extent of functional-anatomical correspondence. The proposed procedure is easy to implement and at no additional measurement costs and should therefore be the method of choice when individual functional data are not available.

The final two chapters of this thesis investigate the non-neural side effects of TMS which can be particularly problematic in attention research. Common control strategies implicitly assume that the non-neural effects of TMS are unspecific, that is, that their effects are the same for different stimulation sites, time points of stimulation, tasks, or any other TMS parameter. Surprisingly, there is hardly any empirical evidence that this assumption is correct and we set out to challenge this assumption on empirical grounds. In Chapter 5, we investigated the non-neural effects of pre-stimulus TMS on target detection. Specifically, we hypothesized that that the clicking sound of a sham TMS pulse differentially affects performance depending on the location of the coil and the timing of the pulse. In Chapter 6 , we investigated the time-dependence of the non-neural effects of TMS for pre- and post-stimulus time windows and their task-dependence. Furthermore, we compared 
real and sham TMS over vertex in order to dissociate the effect of the clicking sound of the TMS coil from the added influence of the somato-sensory effect of TMS. Taken together, these studies challenge the assumption of non-specificity of the nonneural effects of TMS and help to decide what constitutes a valid control condition. In general terms, we aim to provide an empirical foundation for control strategies in TMS research from which concrete guidelines can be derived. 


\section{References}

Bear, M. F., Connors, B. W., \& Paradiso, M. A. (2001). Neuroscience: Exploring the Brain. Baltimore: Lippincott.

Carrasco, M., Ling, S., \& Read, S. (2004). Attention alters appearance. Nature Neuroscience, 7(3), 308-313.

Corbetta, M., Kincade, M. J., Lewis, C., Snyder, A. Z., \& Sapir, A. (2005). Neural basis and recovery of spatial attention deficits in spatial neglect. Nature Neuroscience, 8(11), 1603-1610.

Corbetta, M., \& Shulman, G. L. (2002). Control of goal-directed and stimulusdriven attention in the brain. Nature Reviews Neuroscience, 3(3), 201-215.

Corbetta, M., \& Shulman, G. L. (2011). Spatial neglect and attention networks. Annual Review of Neuroscience, 34(1), 569-599.

Dambeck, N., Sparing, R., Meister, I. G., Wienemann, M., Weidemann, J., Topper, R., \& Boroojerdi, B. (2006). Interhemispheric imbalance during visuospatial attention investigated by unilateral and bilateral TMS over human parietal cortices. Brain Research, 1072(1), 194-199.

Engel, A. K., Fries, P., \& Singer, W. (2001). Dynamic Predictions: Oscillations and Synchrony in Top-Down Processing. Nature Reviews Neuroscience, 2, 704-716.

Grosbras, M.-H., \& Paus, T. (2002). Transcranial magnetic stimulation of the human frontal eye field: effects on visual perception and attention. Journal of Cognitive Neuroscience, 14(7), 1109-1120.

Grosbras, M.-H., \& Paus, T. (2003). Transcranial magnetic stimulation of the human frontal eye field facilitates visual awareness. European Journal of Neuroscience, 18, $3121-3126$.

Hallett, M. (2000). Transcranial magnetic stimulation and the human brain. Nature, 406(6792), 147-150. 
Heilman, K. M., \& Abell, T. Van Den. (1980). Right hemisphere dominance for attention: The mechanism underlying hemispheric asymmetries of inattention (neglect). Neurology, 30(3), 327-330.

Hilgetag, C. C., Theoret, H., \& Pascual-Leone, A. (2001). Enhanced visual spatial attention ipsilateral to rTMS-induced "virtual lesions" of human parietal cortex. Nature Neuroscience, 4(9), 953-957.

Hippocrates. On the Sacred Disease.

Retrieved from http://classics.mit.edu/Hippocrates/sacred.html

Hopfinger, J. B., Buonocore, M. H., \& Mangun, G. R. (2000). The neural mechanisms of top-down attentional control. Nature Neuroscience, 3(3), 284-291.

James, W. (1890). The Principles of Psychology (pp. 411-412). New York: Henry Holt.

Jensen, O., \& Mazaheri, A. (2010). Shaping functional architecture by oscillatory alpha activity: gating by inhibition. Frontiers in Human Neuroscience, 4, 186.

Kastner, S., Pinsk, M. A., De Weerd, P., Desimone, R., \& Ungerleider, L. G. (1999). Increased activity in human visual cortex during directed attention in the absence of visual stimulation. Neuron, 22(4), 751-761.

Kastner, S., \& Ungerleider, L. G. (2000). Mechanisms of Visual Attention in the Human Cortex. Annual Review of Neuroscience, 23, 315-341.

Kincade, J. M., Abrams, R. A., Astafiev, S. V, Shulman, G. L., \& Corbetta, M. (2005). An event-related functional magnetic resonance imaging study of voluntary and stimulus-driven orienting of attention. The Journal of Neuroscience, 25(18), 4593-4604.

Kinsbourne, M. (1977). Hemi-neglect and hemisphere rivalry. Advances in Neurology, 18, 41-49. 
McAdams, C. J., \& Maunsell, J. H. (1999). Effects of attention on orientation-tuning functions of single neurons in macaque cortical area V4. The Journal of Neuroscience, 19(1), 431-441.

Mesulam, M. M. (1981). A cortical network for directed attention and unilateral neglect. Annals of Neurology, 10(4), 309-325.

Moore, T., Armstrong, K. M., \& Fallah, M. (2003). Visuomotor origins of covert spatial attention. Neuron, 40(4), 671-683.

Pascual-Leone, A., Walsh, V., \& Rothwell, J. (2000). Transcranial magnetic stimulation in cognitive neuroscience - virtual lesion, chronometry, and functional connectivity. Current Opinion in Neurobiology, 10(2), 232-237.

Pearce, J. M. S. (2009). Marie-Jean-Pierre Flourens (1794-1867) and cortical localization. European Neurology, 61(5), 311-314.

Posner, M. I. (1980). Orienting of attention. Quarterly Journal of Experimental Psychology, 32(1), 3-25. Psychology Press.

Posner, M. I., Snyder, C. R., \& Davdison, B. J. (1980). Attention and the detection of signals. Journal of Experimental Psychology, 109(2), 160-174.

Reynolds, J. H., \& Chelazzi, L. (2004). Attentional modulation of visual processing. Annual Review of Neuroscience, 27, 611-647. Annual Reviews.

Reynolds, J. H., Chelazzi, L., \& Desimone, R. (1999). Competitive mechanisms subserve attention in macaque areas V2 and V4. The Journal of Neuroscience, 19(5), $1736-1753$.

Reynolds, J. H., \& Heeger, D. J. (2009). The Normalization Model of Attention. Neuron, 61(2), 168-185. Elsevier Inc.

Reynolds, J. H., Pasternak, T., \& Desimone, R. (2000). Attention increases sensitivity of V4 neurons. Neuron, 26(3), 703-714. 
Rorden, C., \& Karnath, H.-O. (2004). Using human brain lesions to infer function: a relic from a past era in the fMRI age? Nature Reviews Neuroscience, 5(10), 813819.

Sack, A. T. (2006). Transcranial magnetic stimulation, causal structure-function mapping and networks of functional relevance. Current Opinion in Neurobiology, $16(5), 593-599$.

Sack, A. T., Cohen Kadosh, R., Schuhmann, T., Moerel, M., Walsh, V., \& Goebel, R. (2009). Optimizing functional accuracy of TMS in cognitive studies: a comparison of methods. Journal of Cognitive Neuroscience, 21(2), 207-221.

Seyal, M., Ro, T., \& Rafal, R. (1995). Increased sensitivity to ipsilateral cutaneous stimuli following transcranial magnetic stimulation of the parietal lobe. Annals of Neurology, 38(2), 264-267.

Sheremata, S. L., Bettencourt, K. C., \& Somers, D. C. (2010). Hemispheric asymmetry in visuotopic posterior parietal cortex emerges with visual short-term memory load. The Journal of Neuroscience, 30(38), 12581-12588.

Shulman, G. L., Pope, D. L. W., Astafiev, S. V, McAvoy, M. P., Snyder, A. Z., \& Corbetta, M. (2010). Right hemisphere dominance during spatial selective attention and target detection occurs outside the dorsal frontoparietal network. The Journal of Neuroscience, 30(10), 3640-3651.

Silvanto, J., Lavie, N., \& Walsh, V. (2006). Stimulation of the human frontal eye fields modulates sensitivity of extrastriate visual cortex. Journal of Neurophysiology, 96(2), 941-945.

Silvanto, J., Muggleton, N., Lavie, N., \& Walsh, V. (2009). The Perceptual and Functional Consequences of Parietal Top-Down Modulation on the Visual Cortex. Cerebral Cortex, 19(2), 327-330.

Silver, M. A., \& Kastner, S. (2009). Topographic maps in human frontal and parietal cortex. Trends in Cognitive Sciences, 13(11), 488-495. 
Szczepanski, S. M., Konen, C. S., \& Kastner, S. (2010). Mechanisms of spatial attention control in frontal and parietal cortex. The Journal of Neuroscience, 30(1), $148-160$.

Walsh, V., \& Cowey, A. (2000). Transcranial magnetic stimulation and cognitive neuroscience. Nature Reviews Neuroscience, 1(1), 73-79. 


\section{Chapter 2}

Hemispheric Differences in the Voluntary Control of Spatial Attention: Direct Evidence for a Right-Hemispheric Dominance within Frontal Cortex

Based on:

Duecker, F., Formisano, E., Sack, A. T. (2013). Hemispheric Differences in the Voluntary Control of Spatial Attention: Direct Evidence for a Right-Hemispheric Dominance within Frontal Cortex. Journal of Cognitive Neuroscience, 25(8), 13321342. 


\section{Abstract}

Lesion studies in neglect patients have inspired two competing models of spatial attention control, namely Heilman's "hemispatial" theory and Kinsbourne's “opponent processor" model. Both assume a functional asymmetry between the two hemispheres, but propose very different mechanisms. Neuroimaging studies have identified a bilateral dorsal fronto-parietal network underlying voluntary shifts of spatial attention. However, lateralization of attentional processes within this network has not been consistently reported. In the current study, we aimed to provide direct evidence concerning the functional asymmetry of right and left FEF during voluntary shifts of spatial attention. To this end, we applied fMRI-guided neuronavigation to disrupt individual FEF activation foci with a longer-lasting inhibitory patterned transcranial magnetic stimulation (TMS) protocol followed by a spatial cueing task. Our results indicate that right FEF stimulation impaired the ability of shifting spatial attention towards both hemifields, whereas the effects of left FEF stimulation were limited to the contralateral hemifield. These results provide strong direct evidence for right-hemispheric dominance in spatial attention within frontal cortex supporting Heilman's "hemispatial" theory. This complements previous TMS studies that generally conform to Kinsbourne's "opponent processor" model after disruption of parietal cortex and we therefore propose that both theories are not mutually exclusive. 


\section{Introduction}

Voluntary control of spatial attention is an important mechanism that allows prioritizing one location of the visual field in order to focus on relevant incoming sensory information. In healthy human volunteers, brain imaging techniques such as PET and fMRI have identified activity in frontal, parietal, and sub-cortical brain regions during tasks requiring shifts of spatial attention (Corbetta \& Shulman, 2002). After stroke or brain injury, damage to those regions frequently causes spatial neglect, a neurological syndrome characterized by the failure to attend, explore, and act upon the contralesional side of space (Corbetta, Kincade, Lewis, Snyder, \& Sapir, 2005). Spatial neglect is more common and severe after damage to the right hemisphere, suggesting a hemispheric asymmetry of the mechanisms underlying spatial attention (Mesulam, 1981). Based on lesion findings, two models of spatial attention have emerged over the years, both accounting for this asymmetry but proposing very different mechanisms. According to Heilman's "hemispatial" theory, the right hemisphere is dominant in spatial attention, mediating attention shifts to both hemifields whereas the left hemisphere exclusively mediates attention shifts to the contralateral hemifield (Heilman \& Abell, 1980). In contrast, Kinsbourne's "opponent processor" model suggests that each hemisphere biases attention to the contralateral hemifield, with the rightward bias of the left hemisphere being stronger, and that both hemispheres are kept in balance due to inter-hemispheric competition (Kinsbourne, 1977).

Despite the fact that both theories propose very different mechanisms of spatial attention control, neuroimaging studies thus far have failed to provide consistent evidence in favor of either view (Corbetta \& Shulman, 2011). Mostly, bilateral activation is reported in the dorsal fronto-parietal network including the frontal eye field (FEF) and the intraparietal sulcus (IPS), neither supporting the notion of a contralateral bias nor of right-hemispheric dominance (Hopfinger, Buonocore, \& Mangun, 2000; Kastner, Pinsk, De Weerd, Desimone, \& Ungerleider, 1999; Kincade, Abrams, Astafiev, Shulman, \& Corbetta, 2005; Shulman et al., 2010). However, a contralateral bias is sometimes observed (Szczepanski, Konen, \& Kastner, 2010) and topographic representations of the contralateral hemifield within 
the dorsal network (Silver \& Kastner, 2009) lend some support to the "opponent processor" model. Conversely, evidence in favor of the "hemispatial" theory has recently also been reported (Sheremata, Bettencourt, \& Somers, 2010). Beyond these inconsistencies, neuroimaging studies have been criticized on general grounds when assessing functional asymmetries (e.g. Macaluso \& Patria, 2007) and caution is required when inferring the functional role of brain areas based on task-correlated activation alone.

In recent years, transcranial magnetic stimulation (TMS) has proven to be a valuable complementary method for the non-invasive study of structure-function relationships (Sack, 2006). Several studies have already successfully used TMS over parietal and frontal cortex to disrupt attentional processes (for a review, see Chambers \& Mattingley, 2005). Neglect-like symptoms are frequently observed after parietal cortex stimulation and the evidence so far is in favor of Kinsbourne's “opponent processor” model (Dambeck et al., 2006; Hilgetag, Theoret, \& PascualLeone, 2001; Seyal, Ro, \& Rafal, 1995; Silvanto, Muggleton, Lavie, \& Walsh, 2009). For example, Hilgetag et al. (2001) found contralateral impairments and ipsilateral enhancements of target detection after $1 \mathrm{~Hz}$ rTMS over left and right IPS conforming to Kinsbourne's notion of a contralateral bias and inter-hemispheric competition. In accordance with this interpretation, Dambeck et al. (2006) revealed that target detection is unaffected when TMS is applied simultaneously over left and right parietal cortex whereas unilateral TMS impairs detection of contralateral stimuli. Frontal brain areas, on the other hand, have received far less attention but TMS studies generally support an involvement of FEF in tasks with attentional components. TMS over FEF has been shown to affect reorienting of attention (Smith, Jackson, \& Rorden, 2005), inhibition of return (Ro, Farnè, \& Chang, 2003), and visual search (Muggleton, Juan, Cowey, \& Walsh, 2003). However, the functional asymmetry between hemispheres has rarely been addressed and those studies that directly compared the functional role of right and left FEF were unable to dissociate attentional from perceptual effects (Grosbras \& Paus, 2002, 2003; Silvanto, Lavie, \& Walsh, 2006). With this limitation in mind, TMS over frontal areas has produced results that are in favor of Heilman's "hemispatial" theory, contrasting the findings in parietal cortex discussed above. For example, it has been 
shown that right FEF stimulation increases perceptual sensitivity in both hemifields whereas left FEF stimulation effects are limited to the contralateral hemifield (Grosbras \& Paus, 2003). The same pattern of results has been observed in terms of changed phosphene thresholds when assessing the top-down influence of FEF on cortical excitability in extrastriate cortex (Silvanto et al., 2006).

In the current study, we aimed to provide direct evidence concerning the functional role of, and hemispheric asymmetry between, left and right FEF during voluntary spatial attention shifts that may differentiate between competing models of spatial attention control. To this end, we used fMRI to first identify right and left FEF in each out of twenty healthy participants, and then applied fMRI-guided neuronavigation to disrupt individual FEF activation foci with a longer-lasting inhibitory patterned TMS protocol. Following these experimentally-induced "virtual lesions", participants were required to perform a spatial cueing task specifically designed to trigger covert spatial attention shifts and to separate orienting from reorienting of attention.

\section{Materials and Methods}

\section{Participants}

Twenty participants (14 female, 6 male; aged 19-28) were recruited from the Maastricht University community. All were right-handed, had normal or correctedto-normal vision and had no history of neurological or psychiatric illness. Written informed consent was obtained prior to participation and the study was approved by the local medical-ethical committee. Additionally, each participant was screened for fMRI and TMS experimentation safety by an independent medical supervisor.

\section{Stimuli and Task}

Participants performed a detection task with central symbolic cues (Fig. 1) prompting covert shifts of attention to the expected target location (Posner, 1980). A 
fixation cross was continuously presented at the centre of the screen. Cues consisted of two arrowheads flanking the fixation cross and pointed either to the left $(<+<)$, right $(>+>)$, or in opposite directions $(\langle+>)$. Gabor patches (spatial frequency $=1.5$ cycles per degree; envelope standard deviation $=0.75$ degrees; Michelson contrast $=60 \%$; random orientations) were used as target stimuli and presented either left or right of the fixation cross at 7 degrees eccentricity. Participants were instructed to press the space bar on a standard keyboard with the right index finger as fast as possible whenever a target was detected irrespective of target location and orientation. Catch trials without a target stimulus were included to prevent anticipatory responses. Stimuli were presented on a gamma-corrected 17" TFT screen (Samsung SyncMaster $931 \mathrm{DF}$ ) at $57 \mathrm{~cm}$ viewing distance. The video mode was $1280 \times 1024$ at $60 \mathrm{~Hz}$ and background luminance was $25 \mathrm{~cd} / \mathrm{m}^{2}$. The Presentation software package (NeuroBehavioural Systems, Albany, CA) was used to control stimulus presentation and recording of behavioral responses.

The task consisted of nine conditions that resulted from the combination of three cues (left, right, neutral) and three target locations (left, right, none/catch). Trials in which the cue provided valid information about the target location were more

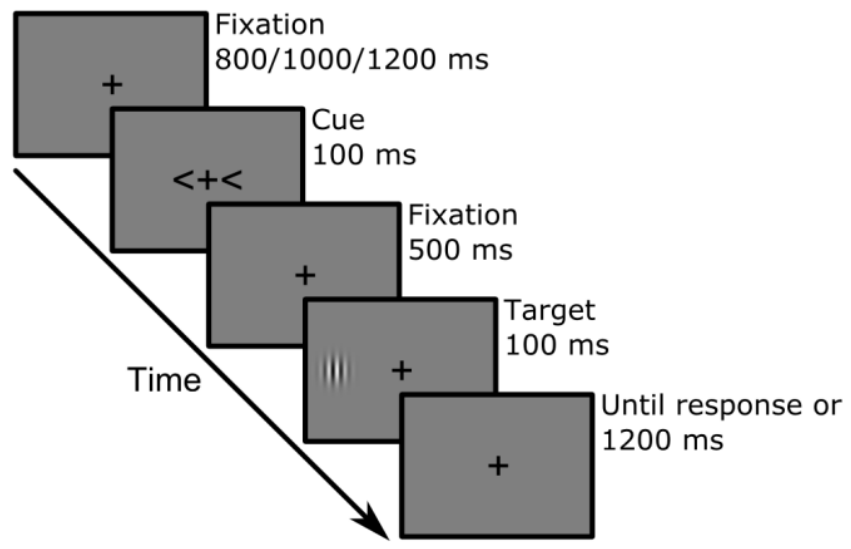

Figure 1. The sequence and timing of events for one possible trial is shown. In this case, the cue points to the left, prompting the participant to covertly shift attention to the left hemifield, and the target appears at the cued location (valid trial). A description of the other cue/target combinations is given in the main text. 
frequent than invalid trials ( $80 \%$ predictive validity) so that utilization of the cue was behaviorally beneficial. In neutral trials, the cue provided no information about the target location. Conceptually, neutral trials serve as a baseline condition that matches valid and invalid trials in terms of perceptual processing, target detection, and the behavioral response but lacks attentional components based on spatial expectancies. Trials with directional cues, however, allow covert shifts of spatial attention to the expected target location. In case of valid trials, reaction times are typically faster compared to neutral trials (attentional benefits) indicating more efficient processing due to successful voluntary attention shifts. In case of invalid trials, reaction times are typically slower compared to neutral trials (attentional costs) indicating that incorrect information about the target location requires reorienting of attention to the unexpected target location after target appearance. Taken together, this task isolates different attentional processes in a well-controlled way and allows measuring these processes in terms of reaction time differences between neutral and valid/invalid trials.

Each trial started with a cue presented for $100 \mathrm{~ms}$ indicating where the target stimulus was most likely to appear. At $600 \mathrm{~ms}$ after cue onset, the target stimulus was presented for $100 \mathrm{~ms}$ followed by a $1200 \mathrm{~ms}$ response window. The trial was terminated immediately after a button press and there was an inter-trial interval of either 800, 1000, or $1200 \mathrm{~ms}$. Participants received corrective feedback (an error message on the screen) in case of false alarms, misses, anticipatory responses (RT below $100 \mathrm{~ms}$ or button press within the cue-target interval), or very slow responses (RT above $800 \mathrm{~ms}$ ).

\section{Functional Magnetic Resonance Imaging}

Anatomical and functional data were acquired for each participant to allow precise localization of individual stimulation sites. The frontal eye field localizer consisted of a simple block design with alternating blocks of central fixation and saccadic eye movements. During saccadic eye movement blocks, every $500 \mathrm{~ms}$ a circle appeared at one out of eight pre-defined locations along the horizontal or vertical meridian and participants were instructed to track these circles with the eyes. In total, 
participants completed 21 blocks each lasting 16 seconds. Even though the present study focused on the role of the FEF in spatial attention, we have chosen for localization based on eye movements. Conceptually, this is closer to the original definition of the FEF (for an historical overview, see Amiez \& Petrides, 2009). Moreover, eye movement-related BOLD signal changes in FEF are more robust compared to attention-related activation changes allowing easier and more time efficient localization (De Haan, Morgan, \& Rorden, 2008). Several studies have confirmed the involvement of the FEF in both spatial attention and eye movements and neuroimaging studies generally report overlapping clusters of activation (Corbetta et al., 1998; de Haan et al., 2008).

Imaging was performed with a Siemens Allegra 3.0 Tesla scanner equipped with a standard head coil (Siemens Medical Systems, Erlangen, Germany). Highresolution anatomical images covering the whole head were collected with a T1weighted 3D ADNI MP-RAGE sequence (192 sagittal slices; matrix = $256 \times 256$; field of view $=256 \times 256 \mathrm{~mm}$; slice thickness $=1 \mathrm{~mm}$; no gap; in-plane voxel size $=1 \times 1 \mathrm{~mm}$; flip angle $=9^{\circ}$; repetition time $=2250 \mathrm{~ms}$; echo time $=2.6 \mathrm{~ms}$ ) . Functional images were obtained using a T2*-weighted single shot echo-planar imaging (EPI) sequence (32 oblique slices with full-brain coverage; matrix $=64 \mathrm{x}$ 64; field of view $=192 \times 192 \mathrm{~mm}$; slice thickness $=3 \mathrm{~mm}$; no gap; in-plane voxel size $=3 \times 3 \mathrm{~mm}$; flip angle $=90^{\circ}$; repetition time $=2000 \mathrm{~ms}$; echo time $=30 \mathrm{~ms}$ ) Visual stimuli were back-projected on a screen at the rear end of the scanner bore and could be seen by the participants via a mirror system attached to the head coil.

Data were pre-processed and analyzed using the BrainVoyager QX 2.3 software package with default settings (Brain Innovation, Maastricht, The Netherlands). Anatomical data were corrected for intensity inhomogeneities and transformed to Talairach space (Talairach \& Tournoux, 1988). Then, a reconstruction of the cortical surface was created for both hemispheres to support TMS coil positioning by improving the visualization of the anatomical gyrification. The first two volumes of the functional data were discarded because of T1 saturation effects. Pre-processing steps included slice scan time correction, 3D motion correction, and high-pass filtering $(2$ cycles). Then, functional data were co-registered with the anatomical data. Finally, the general linear model was used for statistical analysis and the 

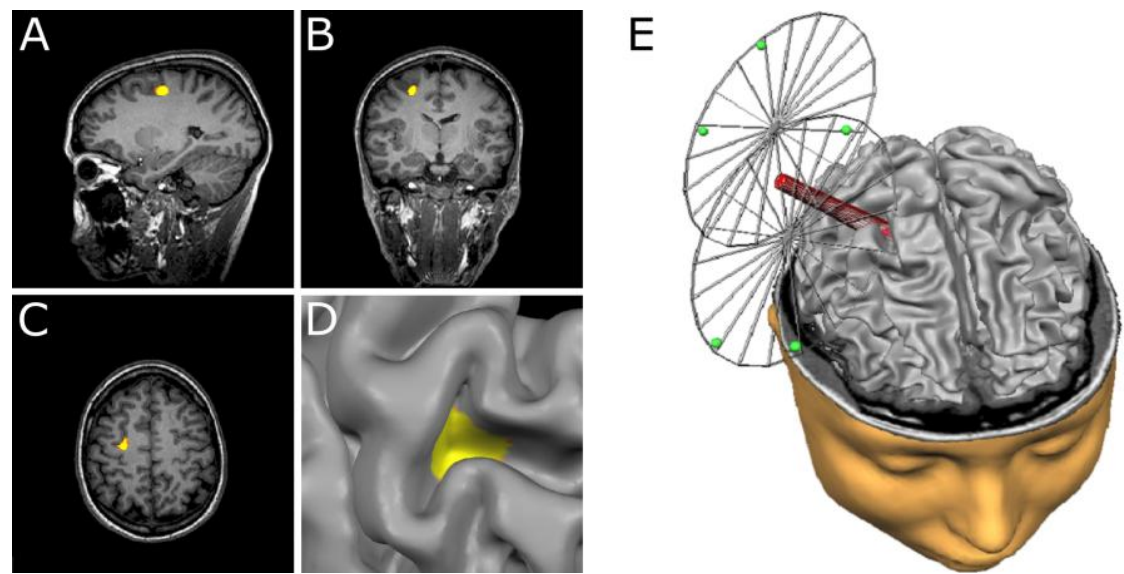

Figure 2. Panels $\mathrm{A}, \mathrm{B}$, and $\mathrm{C}$ show the activation cluster of the right FEF for one representative participants in a sagittal, coronal, and transverse section of the brain, respectively. Panel D shows the same cluster projected on a reconstruction of the cortical surface with the central sulcus on the left and the FEF at the junction of the precentral sulcus and superior frontal sulcus. Panel E shows a snapshot of the neuronavigation system used to guide TMS coil positioning in real-time with the red beam indicating where the magnetic field is strongest.

frontal eye field was identified as the most significant cluster around the precentral sulcus and superior frontal sulcus in each hemisphere (see Fig. $2 A-D$ for an example). No further group analysis was performed because the localizer was used to identify activation foci for each participant in order to guide TMS coil positioning on an individual basis (Fig. 2E).

\section{Transcranial Magnetic Stimulation}

Transcranial magnetic stimulation was applied using a Medtronic MagPro X100 stimulator (Medtronic Functional Diagnostics A/S, Skovlunde, Denmark) and a figure-of-eight TMS coil (MC-B70; inner radius $=10 \mathrm{~mm}$; outer radius $=50 \mathrm{~mm}$ ). During each TMS session, continuous theta burst stimulation was applied for 40 seconds (600 pulses; $50 \mathrm{~Hz}$ triplets in a $5 \mathrm{~Hz}$ rhythm) over one of the three stimulation sites. This protocol has previously been shown to decrease cortical excitability and is commonly thought to have long lasting effects of up to one hour (Huang, Edwards, Rounis, Bhatia, \& Rothwell, 2005). Stimulation intensity was set 
at $80 \%$ of the individual resting motor threshold (mean: $26.4 \%$ of maximum stimulator output; range $19 \%$ - $37 \%$ ). The motor threshold was determined using single pulse TMS over the right motor cortex. It was defined as the smallest intensity that elicited a visible muscle twitch in the contralateral index finger in three out of six trials. None of the participants reported any adverse side effects of the stimulation.

The BrainVoyager TMS Neuronavigator system (BrainInnovation, Maastricht, The Netherlands) was used to ensure precise coil positioning (Fig. 2E). This frameless stereotactic system allows real-time visualization of the TMS coil relative to individual brain anatomy and functionally defined target points based on fMRI data. We used this system to navigate the TMS coil to the optimal position for targeting individual activation foci in each participant. The TMS coil was manually held perpendicular to the skull and the distance between the centre of the coil and target point was kept as small as possible. For the left and right frontal eye field, the coil was oriented parallel to the precentral sulcus with the coil handle pointing lateral. In case of vertex stimulation, the coil was oriented in line with the longitudinal fissure and the coil handle pointed posterior.

\section{Procedure and Design}

Prior to the main experiment, all participants completed a pilot session that was identical to subsequent sessions with the exception that no TMS was applied. The purpose of this session was to assess whether participants made use of the information provided by the cues. Participants were only invited for the remaining experiment in case of statistically significant cueing effects. More specifically, we checked for the presence of attentional benefits and costs indicating shifts of spatial attention after the cue and reorienting of spatial attention after target appearance at an unexpected location, respectively. The task was performed as expected by 20 participants and only one participant was excluded because cues were ineffective in modulating behavior.

We used a full within-subject design so that all participants received TMS to the right FEF, left FEF, and vertex. Vertex stimulation was considered a control 
condition where no effects on task performance were expected. All TMS sessions were identical except for the stimulation site and the order of sessions was counterbalanced across participants. In order to prevent carry-over effects, TMS sessions were separated by at least one day without TMS. During TMS sessions, participants were comfortably seated in a chair with the head supported by a chin rest. TMS was applied after completion of 72 practice trials, immediately followed by the spatial attention task. In total, participants completed 10 blocks each consisting of 72 trials presented in randomized order. Including short breaks between blocks, the overall duration of the behavioral task never exceeded 35 minutes. The proportion of valid:invalid:neutral:catch trials in each experimental block was 8:2:5:3, resulting in 320 valid trials, 80 invalid trials, 200 neutral trials, and 120 catch trials per TMS session. At the beginning of a block, four additional practice trials were included that were not considered in the analysis. Between blocks, participants received feedback about their average performance to ensure that they were motivated throughout the session.

\section{Eye Movement Control}

Electrooculography (EOG) was used during task execution to allow offline control of fixation performance. Data were recorded bipolarly from two pairs of $\mathrm{Ag} / \mathrm{AgCl}$ electrodes with a BrainAmp ExG system (BrainProducts GmbH, Munich, Germany). Eye movements and eye blinks were monitored with electrodes positioned at the outer canthus of each eye (horizontal EOG) and above and below the right eye (vertical EOG). Additionally, a reference electrode was placed on the mastoid behind the right ear. The impedance of all electrodes was kept below $5 \mathrm{k} \Omega$. The EOG signal was digitized at $1000 \mathrm{~Hz}$, high-pass filtered at $0.1 \mathrm{~Hz}$, and stored on disk using BrainVision Recorder (BrainProducts GmbH, Munich, Germany). Offline data analysis was performed with BrainVision Analyzer (BrainProducts $\mathrm{GmbH}$, Munich, Germany). Single trial data were visually inspected and all trials contaminated by eye movements or eye blinks between cue appearance and button press were excluded from further analysis. This was necessary because eye movements indicate that participants potentially failed to covertly shift their 
attention to the cued location and eye blinks can interfere with the perception of the cue and target.

\section{Statistical Analysis}

Two participants were excluded from the analysis because of excessive eye movements (more than $20 \%$ of all trials). For the remaining eighteen participants, all trials contaminated by eye movements or eye blinks were discarded. Additionally, trials were regarded as incorrect and removed in case of false alarms, misses, anticipatory responses, or very slow responses. Because participants received corrective feedback after incorrect responses, subsequent trials were excluded as well to allow reconcentration on the task. Trials following invalid trials were removed to counteract sequence effects. Benefits and costs have been shown to be reduced after invalid trials due to strategic changes in the utilization of the cue (Jongen \& Smulders, 2007). Outliers were excluded according to the $1.5 \mathrm{x}$ IQR (interquartile range) criterion. After application of these exclusion criteria, $78 \%$ of all trials remained and were used to compute individual median reaction times. Across all conditions, the proportion of excluded trials never dropped below $75 \%$ and there were no systematic differences between conditions. On the individual level, median reaction times were always based on at least 20 trials per condition. Individual median reaction times were submitted to a three-way repeated-measures ANOVA with stimulation site (right FEF, left FEF, vertex), cue validity (valid, neutral, invalid), and hemifield (left, right) as within-subject factors. Post-hoc pairwise comparisons were corrected for multiple comparisons by Fisher's LSD procedure. 


\section{Results}

\section{FMRI-Based Localization of TMS Target Points}

All participants completed a functional localizer during an fMRI session in order to precisely identify the right and left FEF on an individual basis. This approach accounts for inter-individual differences in FEF localization and allows optimal TMS coil positioning even when functional regions do not correspond to the macroanatomical organization of the brain. Data for the right FEF of one representative participant are shown in Figure 2 together with an illustration of the neuronavigation system used to monitor the exact coil position in real-time.

In order to allow comparisons with other studies, we transformed individual target points to Talairach space (Fig. $3 B$ ) and calculated the average coordinates of the right FEF $(28,-8,48)$ and left FEF $(-25,-8,50)$. These were very similar to FEF coordinates reported in previous neuroimaging studies (Kincade et al., 2005; Shulman et al., 2009). Moreover, the variability of target points across participants was assessed in terms of their Euclidian distance from the average target point (Fig. $3 A$ and $3 C$ ). The average displacement across all participants was $7 \mathrm{~mm}$ for the right
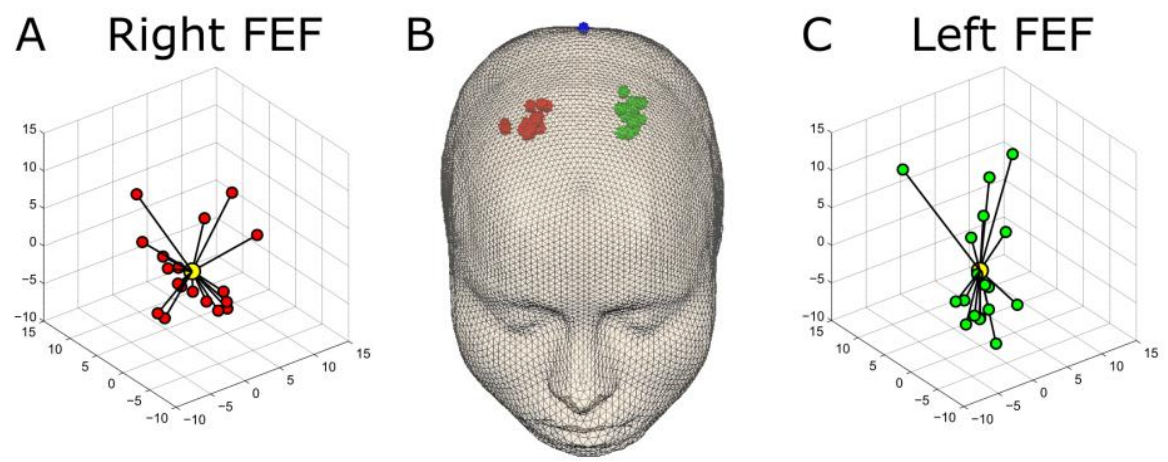

Figure 3. Panel B depicts TMS target points projected on a mesh of a reconstructed head in Talairach space. For the right and left FEF, individual target points are shown in red and green, respectively. For the vertex condition, only the average target point is shown in blue. Panels A and C illustrate deviations from the average target point across participants for the right and left FEF, respectively. In both 3D plots, data are centered around its mean (yellow) and individual target points are plotted according to their displacement in the $\mathrm{x}, \mathrm{y}$, and $\mathrm{z}$ direction in Talairach space on a millimeter scale. 
FEF and $8 \mathrm{~mm}$ for the left FEF with deviations ranging from $2 \mathrm{~mm}$ to $15 \mathrm{~mm}$ for both stimulation sites. Taken together, this indicates that although individual target points were closely clustered, inter-individual differences in FEF localization were notable and likely of practical relevance when it comes to precise application of TMS (Sack et al., 2009).

\section{Errors and Eye Movements}

Participants were able to perform the task at high levels of accuracy during all sessions. The mean accuracy was $98.0 \%, 97.8 \%$, and $98.1 \%$ after stimulation of the right FEF, left FEF, and vertex, respectively. A repeated-measures ANOVA confirmed that accuracy did not differ significantly between sessions $\left(F_{(2,34)}=0.249\right.$, $p>$.70). Similarly, the occurrence of eye movements and eye blinks was unaffected by $\operatorname{TMS}\left(F_{(2,34)}=2.102, p=.138\right)$. Only $5.5 \%$ of all trials were discarded due to these breaks of central fixation with mean values of $6.2 \%, 5.5 \%$, and $4.9 \%$ for right FEF, left FEF, and vertex stimulation, respectively. Most importantly, this indicates that our results are not confounded by effects of frontal eye field stimulation on eye movement control.

\section{Effect of TMS on Reaction Times}

A repeated-measures ANOVA on median reaction times (see Table 1) with stimulation site (right FEF, left FEF, vertex), cue (valid, neutral, invalid), and hemifield (left, right) as within-subject factors revealed significant main effects of cue $\left(F_{(2,34)}=55.390, p<.0001\right)$ and hemifield $\left(F_{(1,17)}=10.119, p<.01\right)$. The main effect of cue resulted from the typical orienting effects observed in spatial cueing tasks. In order to obtain an unbiased estimate of task performance under baseline conditions, we then statistically tested the effect of cue in the vertex condition, undistorted by potential effects of FEF stimulation (Fig. 4). Compared to neutral 
Table 1. Reaction times (in milliseconds) and standard error of mean (in parentheses) for each experimental condition

$$
\text { Left hemifield Right hemifield }
$$

\begin{tabular}{lllllll}
\cline { 2 - 5 } TMS & Right FEF & Left FEF & Vertex & Right FEF & Left FEF & Vertex \\
\hline Valid & $267(7.3)$ & $260(5.7)$ & $261(9.1)$ & $264(7.0)$ & $259(6.9)$ & $256(8.9)$ \\
Neutral & $283(7.2)$ & $280(7.1)$ & $282(9.5)$ & $275(7.6)$ & $268(6.4)$ & $271(9.6)$ \\
Invalid & $315(8.4)$ & $312(9.7)$ & $305(11.2)$ & $305(7.3)$ & $300(9.2)$ & $304(10.9)$ \\
\hline Benefits & $15.4(3.0)$ & $20.4(4.0)$ & $20.9(3.2)$ & $11.2(2.2)$ & $9.1(2.4)$ & $15.1(2.5)$ \\
Costs & $32.4(4.9)$ & $31.2(5.1)$ & $22.3(4.2)$ & $29.7(4.8)$ & $32.7(6.1)$ & $32.5(5.7)$
\end{tabular}

trials, faster reaction times were observed on valid trials (benefits, $t_{(17)}=7.450$, $p<.0001)$ and slower reaction times were observed on invalid trials (costs, $\left.t_{(17)}=5.961, p<.0001\right)$. This strongly indicates that participants made correct use of the cues by performing covert voluntary shifts of spatial attention towards the cued location. Moreover, the presence of both benefits and costs allows segregating different sub-processes of attentional control and is therefore a prerequisite for investigating differential effects of TMS on attentional orienting and reorienting, respectively. The main effect of hemifield resulted from generally faster reaction times for stimuli in the right hemifield. Given that participant always had to respond with their right hand, this most likely is an instance of the Simon effect (Simon \& Rudell, 1967). Most importantly, we also found a significant three-way interaction between stimulation site, cue, and hemifield $\left(F_{(4,68)}=2.748, p<.05\right)$, which was then further explored in terms of benefits (difference in reaction time between neutral and valid trials) and costs (difference in reaction time between neutral and invalid trials) within each hemifield for all stimulation sites. The main effect of stimulation site and the two-way interactions were not significant because of this higher-order interaction. 


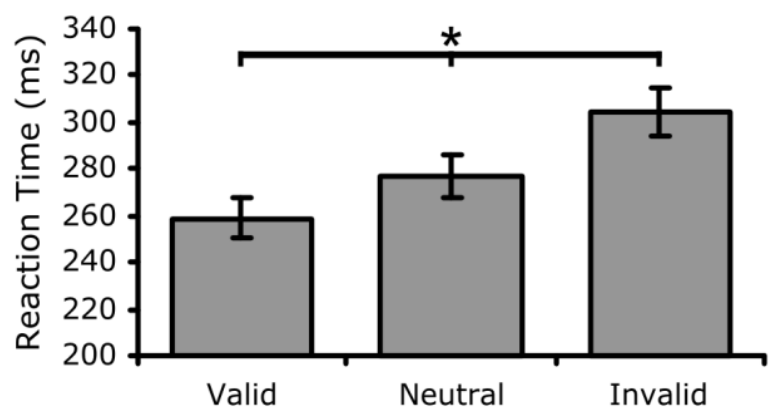

Figure 4. Reaction time data for the vertex condition showed the expected effect of cue validity with robust attentional benefits and costs. All pairwise comparisons are statistically significant at an alpha level of 0.0001 and error bars depict standard errors.

\section{Effect of TMS on Benefits}

We first examined the effects of right and left FEF stimulation on attentional benefits (see Table 1) compared with vertex stimulation with paired $t$-tests. After right FEF stimulation (Fig. 5A), we found a significant reduction of benefits in the left hemifield $\left(t_{(17)}=2.585, p<.05\right)$ as well as in the right hemifield $\left(t_{(17)}=2.257\right.$, $p<.05$ ). After left FEF stimulation (Fig. $5 B$ ), however, we only found a significant reduction of benefits in the right hemifield $\left(t_{(17)}=2.304, p<.05\right)$. Benefits in the left hemifield were unaffected by TMS over left FEF $\left(t_{(17)}=0.140, p>.80\right)$. In sum, both right and left FEF stimulation impaired the ability of shifting spatial attention towards the respective contralateral hemifield, as measured by significantly reduced attentional benefits. However, only TMS over right FEF also significantly impaired spatial attention shifts towards the ipsilateral right hemifield, and thus resulted in bilateral spatial deficits, whereas the effects of left FEF stimulation were limited to the contralateral hemifield. 
A

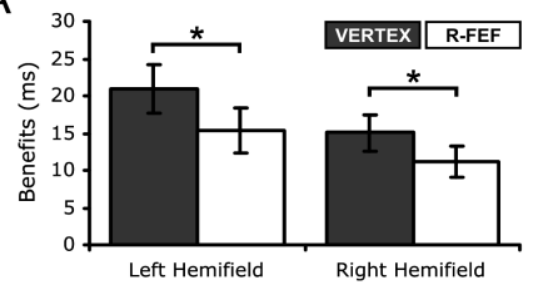

B

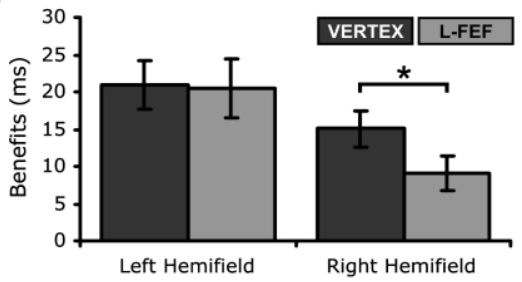

Figure 5. Attentional benefits for each hemifield after TMS over right FEF (panel A) and left FEF (panel B) compared with vertex stimulation. Attentional benefits are defined as the difference in reaction time between neutral and valid trials. Reduced benefits indicate impaired shifts of spatial attention. Differences marked with an asterisk are statistically significant at an alpha level of 0.05 and error bars depict standard errors.

A

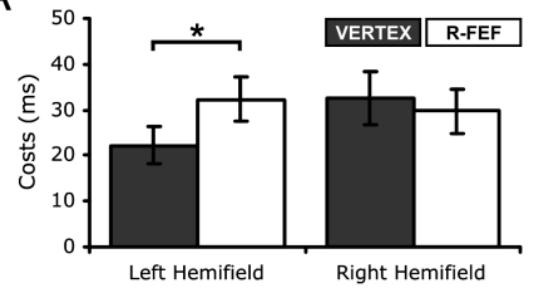

B

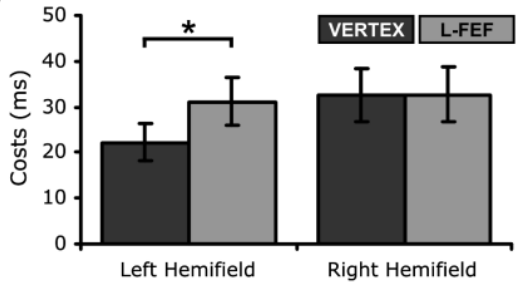

Figure 6. Attentional costs for each hemifield after TMS over right FEF (panel $A$ ) and left FEF (panel $B$ ) compared with vertex stimulation. Attentional costs are defined as the difference in reaction time between neutral and invalid trials. Increased costs indicate impaired reorienting of spatial attention. Differences marked with an asterisk are statistically significant at an alpha level of 0.05 and error bars depict standard errors.

\section{Effect of TMS on Costs}

We then examined the effects of left and right FEF stimulation on attentional costs (see Table 1) compared with vertex stimulation. After right FEF stimulation (Fig. 6A), we found a significant increase of costs in the left hemifield $\left(t_{(17)}=2.175\right.$, $p<.05)$ but not in the right hemifield $\left(t_{(17)}=0.520, p>.60\right)$. After left FEF stimulation (Fig. 6B), we found the same pattern of results with increased costs only in the left hemifield $\left(t_{(17)}=2.879, p<.05\right)$ and no effect in the right hemifield $\left(t_{(17)}=0.044, p>.90\right)$. In sum, both right and left FEF stimulation impaired the 
ability of reorienting spatial attention to the unexpected target location but the effects were limited to the left hemifield.

\section{Effect of TMS on Neutral Trials}

Finally, we examined the effects of left and right FEF stimulation on neutral trials compared with vertex stimulation. A repeated-measures ANOVA with stimulation site and hemifield as within-subject factors revealed a significant main effect of hemifield $\left(F_{(1,17)}=15.134, p<.005\right)$ resulting from generally faster reaction times for stimuli in the right hemifield. However, there was no main effect of $\operatorname{TMS}\left(F_{(2,34)}=0.436, p>.60\right)$ and no significant TMS by hemifield interaction $\left(F_{(2,34)}=1.335, p>.20\right)$. This indicates that the effects of TMS on benefits and costs were contingent on spatial expectations based on directional cues and do not reflect a general impairment in target detection.

\section{Discussion}

Voluntary spatial attention describes our ability to select one location of the visual field to enhance processing of relevant incoming sensory information. In healthy volunteers, neuroimaging studies have identified FEF and intraparietal sulcus as core nodes of a bilateral dorsal fronto-parietal network underlying spatial attention shifts. Accordingly, stroke and brain injuries within these regions are associated with spatial neglect, a neurological syndrome characterized by impaired attentional processes (Corbetta et al., 2005). Because spatial neglect is more common after damage to the right hemisphere, a functional asymmetry in the control of spatial attention between the two hemispheres is widely assumed although the underlying mechanisms are still hotly debated (Corbetta \& Shulman, 2002; Szczepanski et al., 2010). However, neuroimaging studies thus far have failed to consistently reveal lateralization of attentional processes within the dorsal fronto-parietal network (Corbetta \& Shulman, 2011). In recent years, TMS has proven to be a valuable 
complementary method for the non-invasive study of structure-function relationships (Sack, 2006). Using a "virtual lesion" approach, the goal of the present study was to investigate the role and functional asymmetry of right and left FEF during voluntary shifts of spatial attention. We used a spatial cueing task specifically designed to trigger covert spatial attention shifts and assessed the behavioral consequences of right and left FEF disruption. A longer-lasting inhibitory patterned TMS protocol was applied in order to mimic a real lesion in healthy volunteers while benefiting from fMRI-guided neuronavigation allowing precise targeting of individual activation foci.

Using this approach of experimental perturbation, the present study revealed that only disruption of right FEF causes a reduction of attentional benefits in both hemifields whereas disruption of left FEF exclusively affects attentional benefits in the contralateral hemifield. This is, to the best of our knowledge, the first direct experimental evidence supporting the general notion of functional asymmetry between right and left frontal cortex in spatial attention as originally proposed by Heilman (1980) and Mesulam (1981). Furthermore, our findings bear direct implications for current functional-anatomical models of spatial attention which have been discussed controversially in a recent review (Corbetta \& Shulman, 2011). While the involvement of FEF in voluntary control of spatial attention is undisputed (Corbetta \& Shulman, 2002), there is an ongoing debate between two competing theories of spatial attention control, namely Heilman's "hemispatial" theory (Heilman \& Abell, 1980) and Kinsbourne's “opponent processor" model (Kinsbourne, 1977).

Our results provide strong direct evidence for right-hemispheric dominance in spatial attention supporting Heilman's "hemispatial" theory. According to this view, the right hemisphere mediates attention shifts to both hemifields whereas the left hemisphere only mediates attention shifts to the contralateral right hemifield (Heilman \& Abell, 1980). This functional asymmetry, as predicted by the model, completely matches the pattern of reduced attentional benefits reported here. In contrast, our results are at odds with Kinsbourne's “opponent processor" model which claims that each hemisphere biases attention to the contralateral hemifield (Kinsbourne, 1977). According to this view, a bilateral impairment, as observed 
after right FEF disruption, is unexpected and cannot be readily explained. At first glance, our results are therefore contrasting previous TMS findings that provided evidence in favor of Kinsbourne's model after disruption of parietal cortex (Dambeck et al., 2006; Hilgetag et al., 2001; Seyal et al., 1995; Silvanto et al., 2009). Although this might be perceived as inconsistent, the present findings rather indicate that parietal and frontal cortex have different functional properties. In this sense, Heilman's "hemispatial" theory and Kinsbourne's "opponent processor" model might not be mutually exclusive but rather apply to different nodes of the dorsal fronto-parietal network. The current findings, together with previous TMS studies, could lead the discussion for a combined model of spatial attention control that incorporates mechanisms of inter-hemispheric competition and righthemispheric dominance.

It is noteworthy that the behavioral consequences of FEF stimulation reported here do not fully match the attentional deficits commonly observed in spatial neglect (Mesulam, 1981). More specifically, left hemisphere damage often leaves attentional processes largely intact presumably because the right hemisphere is still capable of shifting attention to both hemifields. Similarly, after right hemisphere damage only attentional deficits are observed in the left hemifield presumably because the intact left hemisphere is still capable of shifting attention to the right hemifield. These compensatory abilities due to overlap in function were not observed with the "virtual lesion" approach used in the present study. While this mismatch might be perceived as an argument against the validity of the "virtual lesion" approach, it actually is a consequence of several advantages of the direct experimental disruption by TMS in healthy volunteers compared with lesion studies. First, behavioral deficits in neglect patients are normally assessed weeks after occurrence of the lesion (e.g. Corbetta et al., 2005) allowing for functional reorganization and compensatory processes, whereas TMS is applied immediately prior to task performance. Second, lesions are hardly ever restricted to one functional brain area and are subject to large interindividual variability (e.g. Mort et al., 2003). In contrast, TMS with fMRI-guided neuronavigation allows localization and targeting of individually defined functional brain areas (Sack et al., 2009). Third, TMS-induced disruption of normal brain functioning is limited in time so that full within-subject designs are possible where 
different "virtual lesions" can be studied in the same participants. Taken together, the "virtual lesion" approach overcomes several confounding factors inherent to lesion studies and can therefore be considered an elegant experimental approach to unmask the real functional role of brain areas in an undistorted way. Only under these conditions, it was possible to reveal the functional asymmetry between right and left FEF and to demonstrate right-hemispheric dominance in spatial attention.

It has to be emphasized that TMS specifically impaired attentional processes. Reaction times on neutral trials were unaffected by TMS which rules out explanations in terms of general changes in target detection or perceptual effects due to modulations of visual cortex. This is in contrast with earlier studies that also investigated the behavioral consequences of FEF stimulation on spatial attention and target detection (Grosbras \& Paus, 2002, 2003). Using a similar spatial cueing task with single pulse TMS prior to target appearance, bilateral effects after right FEF stimulation and contralateral effects after left FEF stimulation were reported, resembling the current findings (Grosbras \& Paus, 2002). However, instead of disruption of attentional processes, a general facilitation across all cueing conditions was found but attentional benefits were unaffected. While this is an interesting finding in itself, such unspecific effects do not allow drawing conclusions about voluntary shifts of spatial attention, as they occur in a spatial cueing task, because attention shifts are generally operationalized in terms of attentional benefits, that is, the difference between valid and neutral trials. A follow-up study requiring detection of near-threshold visual stimuli indeed confirmed that FEF stimulation improves target detection per se when applied prior to target appearance (Grosbras \& Paus, 2003). This is probably due to top-down modulations of visual cortex, as has been shown by the simultaneous combination of TMS and fMRI (Ruff et al., 2006). Consequently, studies investigating the role of FEF in spatial attention by means of "online" TMS, i.e. applying TMS during task execution, struggle to dissociate attentional from perceptual effects. As demonstrated in the current study, the "virtual lesion" approach can have high functional specificity even though it lacks the temporal specificity of online TMS. Nevertheless, a detailed exploration of FEF functioning over time covering the cueing phase and post-target processing would be 
a welcome addition to the current findings and potentially reveal a level of detail that offline TMS cannot achieve.

We also observed increased attentional costs in the left hemifield after stimulation of right and left FEF. This pattern of results differs from the effects of TMS on attentional benefits described above, and as such certainly highlights the general involvement of FEF not only in voluntary shifts of spatial attention (attentional benefits) but also in reorienting of attention towards an unexpected target location (attentional costs). On first sight, this impairment in task performance might be counter-intuitive because one could expect that the disruption of attentional benefits observed after FEF stimulation also leads to reduced attentional costs. With the initial allocation of attention towards the cued location being weaker, it seems plausible that the costs of reorienting decrease as well. However, such an effect could easily be overruled by a TMS-induced disruption of the reorienting process itself. In this sense, our results indicate that attentional costs are not simply the flip side of attentional benefits and that FEF stimulation differentially affects both processes. This interpretation is supported by pharmacological studies showing a dissociation of attentional benefits and costs (Clark, Geffen, \& Geffen, 1989; Murphy \& Klein, 1998; Witte, Davidson, \& Marrocco, 1997).This being said, we would like to remain rather conservative in drawing further conclusions regarding the specific functional asymmetry of FEF for reorienting spatial attention because of the following reason: every invalid trial consists of an initial shift of attention to the cued location followed by reorienting of attention towards the unexpected target location. The effects of TMS on attentional benefits clearly demonstrate that the initial shift of attention is already impaired by TMS. Any effect of TMS on the reorienting process is therefore potentially confounded by this preceding disruption by TMS or, at least, cannot be dissociated from it based on reaction times alone. Nevertheless, the effects of TMS on attentional benefits and costs do differ from each other suggesting differential involvement of FEF in orienting and reorienting of spatial attention. 
Regarding the interpretation of the present data, one important assumption is that the effects of TMS were confined to the stimulation site. In fact, many studies have established that TMS affects remote brain areas within the same functional network (Bestmann, Baudewig, Siebner, Rothwell, \& Frahm, 2005; Ruff et al., 2006; Sack et al., 2007). In our case, stimulation of FEF might also disrupt other nodes of the dorsal and even ventral fronto-parietal network. However, it has to be emphasized that this possibility does not undermine our finding of functional asymmetry between the left and right hemisphere. Instead, this might only be problematic when it comes to attributing the effects to a particular brain region. While we cannot exclude remote effects of TMS with certainty, it nevertheless seems likely that our behavioral effects are due to direct FEF disruption. To begin with, in contrast to the effects of FEF disruption reported here, TMS over parietal cortex commonly produces effects that are more in line with Kinsbourne's "opponent processor" model (Dambeck et al., 2006; Hilgetag et al., 2001; Seyal et al., 1995; Silvanto et al., 2009). In our view, there are no fundamental differences between the present study and previous work that can account for these diverging results except for the difference in stimulation sites. Consequently, it seems most plausible that the functional asymmetry revealed here is due to directly impaired FEF functioning. Furthermore, according to current functional-anatomical models of spatial attention, a spread of TMS effects to the ventral fronto-parietal network is not expected to have effects on voluntary shifts of spatial attention because this network is thought to mediate stimulus-driven attention (Corbetta \& Shulman, 2002). This view is supported by a recent TMS study that reported intact voluntary shifts of spatial attention despite disruption of the right temporo-parietal junction (Chica, Bartolomeo, \& Valero-Cabré, 2011). Taken together, it seems unlikely that our findings are a result of TMS effects remote from the stimulation sites.

As already pointed out, neuroimaging studies have provided inconsistent results regarding the functional asymmetry of spatial attention within the dorsal frontoparietal network. For that reason, a new framework has recently been put forward to explain the predominance of spatial neglect after right hemisphere damage (Corbetta $\&$ Shulman, 2011). In short, even though the spatial deficits observed in neglect are 
closely related to the functional role of the dorsal network, damage outside this network seems to be their primary cause. The ventral fronto-parietal network is lateralized to the right hemisphere and neglect is therefore considered to originate from there but the dorsal network is affected as well (mainly in the right hemisphere) due to disrupted interactions between the two systems. The right hemisphere is no longer being argued to be dominant in spatial attention mainly because of the weak direct empirical evidence supporting this model in the past two decades. Our results illustrate the strength of non-invasive brain stimulation to reveal structure-function relationships where lesion studies and/or pure activation based approaches might struggle. We show that it is premature to give up on the idea of right-hemispheric dominance in the dorsal network. Together with the strong empirical support for Kinsbourne's “opponent processor” model within parietal cortex, it seems most promising to combine both models and to further investigate functional differences within the dorsal fronto-parietal network.

\section{Acknowledgements}

This work was supported by the European Research Council under the European Union's Seventh Framework Programme (FP7/2007-2013) / ERC Grant agreement (263472 to A.T.S.), and the Netherlands Organization for Scientific Research (40007-230 to A.T.S \& E.F.). We thank our medical supervisor Cees van Leeuwen and independent physician Martin van Boxtel. 


\section{References}

Amiez, C., \& Petrides, M. (2009). Anatomical organization of the eye fields in the human and non-human primate frontal cortex. Progress in Neurobiology, 89(2), 220-230.

Bestmann, S., Baudewig, J., Siebner, H. R., Rothwell, J. C., \& Frahm, J. (2005). BOLD MRI responses to repetitive TMS over human dorsal premotor cortex. Neuroimage, 28(1), 22-29.

Chambers, C. D., \& Mattingley, J. B. (2005). Neurodisruption of selective attention: insights and implications. Trends in Cognitive Sciences, 9(11), 542-550.

Chica, A. B., Bartolomeo, P., \& Valero-Cabré, A. (2011). Dorsal and Ventral Parietal Contributions to Spatial Orienting in the Human Brain. The Journal of Neuroscience, 31(22), 8143-8149.

Clark, C. R., Geffen, G. M., \& Geffen, L. B. (1989). Catecholamines and the covert orientation. Neuropsychologia, 27(2), 131-139.

Corbetta, M., Akbudak, E., Conturo, T. E., Snyder, A. Z., Ollinger, J. M., Drury, H. A., Linenweber, M. R., et al. (1998). A common network of functional areas for attention and eye movements. Neuron, 21(4), 761-773.

Corbetta, M., Kincade, M. J., Lewis, C., Snyder, A. Z., \& Sapir, A. (2005). Neural basis and recovery of spatial attention deficits in spatial neglect. Nature Neuroscience, 8(11), 1603-1610.

Corbetta, M., \& Shulman, G. L. (2002). Control of goal-directed and stimulusdriven attention in the brain. Nature Reviews Neuroscience, 3(3), 201-215.

Corbetta, M., \& Shulman, G. L. (2011). Spatial neglect and attention networks. Annual Review of Neuroscience, 34(1), 569-599.

Dambeck, N., Sparing, R., Meister, I. G., Wienemann, M., Weidemann, J., Topper, R., \& Boroojerdi, B. (2006). Interhemispheric imbalance during visuospatial 
attention investigated by unilateral and bilateral TMS over human parietal cortices. Brain Research, 1072(1), 194-199.

Grosbras, M.-H., \& Paus, T. (2002). Transcranial magnetic stimulation of the human frontal eye field: effects on visual perception and attention. Journal of Cognitive Neuroscience, 14(7), 1109-1120.

Grosbras, M.-H., \& Paus, T. (2003). Transcranial magnetic stimulation of the human frontal eye field facilitates visual awareness. European Journal of Neuroscience, 18, 3121-3126.

De Haan, B., Morgan, P. S., \& Rorden, C. (2008). Covert orienting of attention and overt eye movements activate identical brain regions. Brain Research, 1204, 102111.

Heilman, K. M., \& Abell, T. Van Den. (1980). Right hemisphere dominance for attention: The mechanism underlying hemispheric asymmetries of inattention (neglect). Neurology, 30(3), 327-330.

Hilgetag, C. C., Theoret, H., \& Pascual-Leone, A. (2001). Enhanced visual spatial attention ipsilateral to rTMS-induced "virtual lesions" of human parietal cortex. Nature Neuroscience, 4(9), 953-957.

Hopfinger, J. B., Buonocore, M. H., \& Mangun, G. R. (2000). The neural mechanisms of top-down attentional control. Nature Neuroscience, 3(3), 284-291.

Huang, Y.-Z., Edwards, M. J., Rounis, E., Bhatia, K. P., \& Rothwell, J. C. (2005). Theta burst stimulation of the human motor cortex. Neuron, 45(2), 201-206.

Jongen, E. M. M., \& Smulders, F. T. Y. (2007). Sequence effects in a spatial cueing task: endogenous orienting is sensitive to orienting in the preceding trial. Psychological Research, 71(5), 516-523. Springer Berlin / Heidelberg.

Kastner, S., Pinsk, M. A., De Weerd, P., Desimone, R., \& Ungerleider, L. G. (1999). Increased activity in human visual cortex during directed attention in the absence of visual stimulation. Neuron, 22(4), 751-761. 
Kincade, J. M., Abrams, R. A., Astafiev, S. V, Shulman, G. L., \& Corbetta, M. (2005). An event-related functional magnetic resonance imaging study of voluntary and stimulus-driven orienting of attention. The Journal of Neuroscience, 25(18), 4593-4604.

Kinsbourne, M. (1977). Hemi-neglect and hemisphere rivalry. Advances in Neurology, 18, 41-49.

Macaluso, E., \& Patria, F. (2007). Spatial re-orienting of visual attention along the horizontal or the vertical axis. Experimental Brain Research, 180(1), 23-34.

Mesulam, M. M. (1981). A cortical network for directed attention and unilateral neglect. Annals of Neurology, 10(4), 309-325.

Mort, D. J., Malhotra, P., Mannan, S. K., Rorden, C., Pambakian, A., Kennard, C., \& Husain, M. (2003). The anatomy of visual neglect. Brain, 126(9), 1986-1997.

Muggleton, N. G., Juan, C.-H., Cowey, A., \& Walsh, V. (2003). Human frontal eye fields and visual search. Journal of Neurophysiology, 89(6), 3340-3343.

Murphy, F. C., \& Klein, R. M. (1998). The effects of nicotine on spatial and nonspatial expectancies in a covert orienting task. Neuropsychologia, 36(11), 11031114.

Posner, M. I. (1980). Orienting of attention. Quarterly Journal of Experimental Psychology, 32(1), 3-25. Psychology Press.

Ro, T., Farnè, A., \& Chang, E. (2003). Inhibition of return and the human frontal eye fields. Experimental Brain Research, 150(3), 290-296.

Ruff, C. C., Blankenburg, F., Bjoertomt, O., Bestmann, S., Freeman, E., Haynes, J.D., Rees, G., et al. (2006). Concurrent TMS-fMRI and psychophysics reveal frontal influences on human retinotopic visual cortex. Current Biology, 16(15), 1479-1488. 
Sack, A. T. (2006). Transcranial magnetic stimulation, causal structure-function mapping and networks of functional relevance. Current Opinion in Neurobiology, $16(5), 593-599$.

Sack, A. T., Cohen Kadosh, R., Schuhmann, T., Moerel, M., Walsh, V., \& Goebel, R. (2009). Optimizing functional accuracy of TMS in cognitive studies: a comparison of methods. Journal of Cognitive Neuroscience, 21(2), 207-221.

Sack, A. T., Kohler, A., Bestmann, S., Linden, D. E. J., Dechent, P., Goebel, R., \& Baudewig, J. (2007). Imaging the Brain Activity Changes Underlying Impaired Visuospatial Judgments: Simultaneous fMRI, TMS, and Behavioral Studies. Cerebral Cortex, 17(12), 2841-2852.

Seyal, M., Ro, T., \& Rafal, R. (1995). Increased sensitivity to ipsilateral cutaneous stimuli following transcranial magnetic stimulation of the parietal lobe. Annals of Neurology, 38(2), 264-267.

Sheremata, S. L., Bettencourt, K. C., \& Somers, D. C. (2010). Hemispheric asymmetry in visuotopic posterior parietal cortex emerges with visual short-term memory load. The Journal of Neuroscience, 30(38), 12581-12588.

Shulman, G. L., Astafiev, S. V, Franke, D., Pope, D. L. W., Snyder, A. Z., McAvoy, M. P., \& Corbetta, M. (2009). Interaction of stimulus-driven reorienting and expectation in ventral and dorsal frontoparietal and basal ganglia-cortical networks. The Journal of Neuroscience, 29(14), 4392-4407.

Shulman, G. L., Pope, D. L. W., Astafiev, S. V, McAvoy, M. P., Snyder, A. Z., \& Corbetta, M. (2010). Right hemisphere dominance during spatial selective attention and target detection occurs outside the dorsal frontoparietal network. The Journal of Neuroscience, 30(10), 3640-3651.

Silvanto, J., Lavie, N., \& Walsh, V. (2006). Stimulation of the human frontal eye fields modulates sensitivity of extrastriate visual cortex. Journal of Neurophysiology, 96(2), 941-945. 
Silvanto, J., Muggleton, N., Lavie, N., \& Walsh, V. (2009). The Perceptual and Functional Consequences of Parietal Top-Down Modulation on the Visual Cortex. Cerebral Cortex, 19(2), 327-330.

Silver, M. A., \& Kastner, S. (2009). Topographic maps in human frontal and parietal cortex. Trends in Cognitive Sciences, 13(11), 488-495.

Simon, J. R., \& Rudell, A. P. (1967). Auditory S-R compatibility: The effect of an irrelevant cue on information processing. Journal of Applied Psychology, 51(3), 300-304.

Smith, D. T., Jackson, S. R., \& Rorden, C. (2005). Transcranial magnetic stimulation of the left human frontal eye fields eliminates the cost of invalid endogenous cues. Neuropsychologia, 43(9), 1288-1296.

Szczepanski, S. M., Konen, C. S., \& Kastner, S. (2010). Mechanisms of spatial attention control in frontal and parietal cortex. The Journal of Neuroscience, 30(1), 148-160.

Talairach, J., \& Tournoux, P. (1988). Co-Planar Stereotaxic Atlas of the Human Brain. New York: Thieme.

Witte, E. A., Davidson, M. C., \& Marrocco, R. T. (1997). Effects of altering brain cholinergic activity on covert orienting of attention: comparison of monkey and human performance. Psychopharmacology, 132(4), 324-334. 



\section{Chapter 3}

Hemispheric Differences in the Dorsal and Ventral FrontoParietal Attention Network 


\section{Abstract}

Voluntary control of spatial attention allows selecting relevant aspects of incoming sensory information. There are three competing theories of attentional control that are capable of explaining the pre-dominance of spatial neglect after right hemisphere damage. Each of them proposes hemispheric asymmetries either within the dorsal or ventral fronto-parietal network. Here, we experimentally put current functionalanatomical models to the test by disrupting core nodes of both networks, namely the intraparietal sulcus (IPS) and the temporo-parietal junction (TPJ), in both hemispheres using transcranial magnetic stimulation (TMS). We then assessed the behavioral consequences of this disruption on a spatial cueing task in order to shed new light on the functional mechanism underlying attention control, thereby.

We did not observe any effect of TMS on task performance making it impossible to draw any novel conclusions regarding the functional roles of the dorsal and ventral network. Given the abundance of evidence supporting the involvement of IPS and TPJ in attentional control, this null finding should not be seen as evidence that the stimulated brain areas are not functionally relevant for shifts of spatial attention. Instead we identified two potential sources of variability that contributed to the failure of revealing any significant TMS effects on behavior. First, performance across sessions was quite unstable so that within-subject variability was much higher than the expected effect size. Second, localization of TMS target points based on Talairach coordinates does not guarantee that the functional brain areas of interest were disrupted with TMS in all participants.

The present study failed to explore hemispheric asymmetries in the dorsal and ventral fronto-parietal network due to statistical and methodological reasons. However, the research questions addressed here remain of high relevance for current models of attention control and the reasons for the pre-dominance of spatial neglect after right hemisphere damage remain a relevant and timely research question. 


\section{Introduction}

Current models of attentional control have been mainly influenced by two lines of research. To begin with, neuropsychological studies have revealed that spatial neglect is more common and severe after right hemisphere damage (Corbetta, Kincade, Lewis, Snyder, \& Sapir, 2005). This has widely been interpreted as reflecting some kind of hemispheric asymmetry (Mesulam, 1981) and has inspired two competing theories of attentional control, namely Heilman's "hemispatial" theory and Kinsbourne's “opponent processor" model (Heilman \& Abell, 1980; Kinsbourne, 1977). Heilman's "hemispatial" theory states that the right hemisphere mediates attention shifts to both hemifields whereas the left hemisphere mediates attention shifts only to the right hemifield. Kinsbourne's "opponent processor" models states that each hemisphere biases attention to the contralateral hemifield, with the bias of the left hemisphere being stronger, and that inter-hemispheric competition creates a balance between both hemispheres. Despite the differences between both theories, they explain the behavioral consequences of brain damage in neglect patients equally well.

There has been a huge interest in identifying the brain areas involved in attentional control using neuroimaging techniques hoping that this sheds new light on the underlying functional mechanisms. These neuroimaging studies have indeed identified dorsal and ventral fronto-parietal networks that are active when performing tasks requiring shifts of spatial attention (Corbetta \& Shulman, 2002; Doricchi, Macci, Silvetti, \& Macaluso, 2010; Hopfinger, Buonocore, \& Mangun, 2000; Kastner, Pinsk, De Weerd, Desimone, \& Ungerleider, 1999; Kincade, Abrams, Astafiev, Shulman, \& Corbetta, 2005; Shulman et al., 2010; Szczepanski, Konen, \& Kastner, 2010). The dorsal fronto-parietal network consists of the intraparietal sulcus (IPS) and frontal eye field (FEF) with activation commonly reported in both hemispheres. The ventral fronto-parietal network consists of the temporo-parietal junction (TPJ) and ventral frontal cortex (VFC) with activation commonly reported mainly in the right hemisphere. In functional terms, the dorsal network is directly related to orienting of attention and top-down control of sensory areas whereas the ventral network acts as a circuit breaker of the dorsal network in 
case an unexpected or salient stimulus occurs that requires reorienting of attention.

Based on these neuroimaging and lesion findings, Corbetta and Shulman have recently put forward an alternative explanation for the pre-dominance of spatial neglect after right hemisphere damage (2011). They proposed that the rightlateralization of the ventral network is the critical factor even though the dorsal network mediates core attentional functions. In their view, the ventral and dorsal networks are functionally segregated but they nevertheless interact. Damage to the ventral network therefore also affects the dorsal network essentially leading to dysfunctions in both networks explaining the occurrence of attentional deficits. Functional asymmetries within the dorsal network remain a possibility but are not explicitly defined. In this sense, their explanatory approach is a clear shift away from hemispheric asymmetries within the dorsal network.

In order to directly address the question of functional hemispheric asymmetry in attentional control, several studies have used transcranial magnetic stimulation (TMS) to non-invasively disrupt brain activity in parietal and frontal brain areas. As opposed to neuroimaging studies, these TMS experiments have consistently reported hemispheric asymmetries in the dorsal fronto-parietal network. For parietal cortex, findings generally support Kinsbourne's “opponent processor” model with impaired target detection only observed in the hemifield contralateral to the stimulated hemisphere (Dambeck et al., 2006; Hilgetag, Theoret, \& Pascual-Leone, 2001; Seyal, Ro, \& Rafal, 1995; Silvanto, Muggleton, Lavie, \& Walsh, 2009). For frontal cortex, we have recently shown that right and left FEF have functional roles that are in agreement with Heilman's "hemispatial" theory (Duecker, Formisano, \& Sack, in press). Specifically, we found that a "virtual lesion" to the right FEF impaired shifts of spatial attention to both hemifields whereas left FEF disruption only affected shifts of spatial attention to the contralateral hemifield. These attention effects match earlier findings of perceptual modulations after FEF stimulation (Grosbras \& Paus, 2003; Silvanto, Lavie, \& Walsh, 2006). Taken together, these TMS studies provide strong evidence in favor of functional asymmetries within the dorsal fronto-parietal network. Interestingly, it appears that Heilman's "hemispatial" theory applies to frontal cortex whereas Kinsbourne's “opponent processor” model applies to parietal cortex. 
Taken together, there are currently three competing theories of attentional control that are capable of explaining the pre-dominance of spatial neglect after right hemisphere damage. Importantly, each of these theories predicts hemispheric asymmetries either in the dorsal or ventral fronto-parietal network. Given the success of earlier TMS studies in revealing such functional asymmetries, we set out to put these theories to the test by extending our previous work over frontal cortex. To this end, we essentially copied the experimental design that has shown to be successful before (Duecker et al., 2013) and applied it to posterior regions of the dorsal and ventral fronto-parietal network, namely IPS and TPJ in both hemispheres. Using a full within-subject design, we created "virtual lesions" in these core nodes of the dorsal and ventral network and then assessed the behavioral consequences of TMS on a spatial cueing task that triggers covert shifts of spatial attention and allows separating orienting from reorienting of attention. Importantly, we quantified these processes as reaction time differences between different spatial expectancy states with so called attentional benefits reflecting voluntary shifts of attention mediated by the dorsal network and so called attentional costs reflecting reorienting of attention mediated by the dorsal and ventral network. Based on the theories outlined above, we formulated three research questions that can be used for hypothesis-driven interpretation of the data and are therefore of direct relevance for current functional-anatomical models of attentional control

First, we examined the functional asymmetry within the dorsal network between right and left IPS. TMS studies thus far have supported Heilman's "hemispatial" theory and Kinsbourne's “opponent processor" model for frontal and parietal nodes of the dorsal network, respectively. However, it is still a possibility that these observed functional differences can be accounted for by task-related factors or the specific TMS parameters being used. With these factors being identical to our previous work (Duecker et al., 2013), we here re-examined the functional roles of right and left IPS with attentional benefits being the primary outcome measure. Second, we investigated the right-lateralization of the ventral network as suggested by Corbetta and Shulman (Corbetta \& Shulman, 2002, 2011; Shulman et al., 2010). Even though most neuroimaging studies support the notion of a lateralized ventral network, there are some indications that left TPJ also plays a role in attentional 
processes (Doricchi et al., 2010). To the best of our knowledge, we here used TMS for the first time to assess the functional asymmetry between right and left TPJ with attentional costs being the primary outcome measure. Third, we explored the consequences of disrupting the ventral network on attentional functions associated with the dorsal network. The explanation for the pre-dominance of spatial neglect after right hemisphere damage by Corbetta and Shulman (2011) heavily relies on the assumption that damage to the ventral network causes dysfunction of the dorsal network. Here, we assessed the behavioral consequences of a "virtual lesion" to the ventral network on attentional benefits that are purely associated with the dorsal network; a direct test of their model.

\section{Materials and Methods}

\section{Participants}

Eighteen participants (15 female, 3 male; aged 19-28) were recruited from the student population of Maastricht University. All were right-handed, had normal or corrected-to-normal vision and had no history of neurological or psychiatric illness. The study was approved by the local medial-ethical committee and participants gave written informed consent prior to participation. An independent medical supervisor screened all participants for MRI and TMS experimentation safety.

\section{Stimuli and Task}

As originally described by Posner (Posner, Snyder, \& Davdison, 1980; Posner, 1980), we used a detection task with central symbolic cues (Figure 1) that prompt covert shifts of spatial attention. A fixation cross was continuously presented at the center of the screen and cues consisted of two arrowheads flanking the fixation cross. They pointed either to the left $(<+<)$, right $(>+>)$, or in opposite directions $(<+>)$. Gabor patches served as target stimuli (spatial frequency $=1.5$ cycles per degree; envelope standard deviation $=0.75$ degrees; Michelson contrast $=60 \%$; 
random orientation) and were presented at 7 degrees eccentricity either left or right of the fixation cross. Participants were instructed to indicate target detection by pressing the space bar on a standard keyboard with the right index finger as fast as possible irrespective of target location. In order to prevent anticipatory responses based temporal expectancies, catch trials without a target stimulus were included where participants had to withhold their response. Stimuli were presented on a gamma-corrected 17" TFT screen (Samsung SyncMaster 931 DF) at $57 \mathrm{~cm}$ viewing distance. The video mode was 1280 x 1024 at $60 \mathrm{~Hz}$ and background luminance was $25 \mathrm{~cd} / \mathrm{m}^{2}$. The Presentation software package (NeuroBehavioural Systems, Albany, CA) was used to control stimulus presentation and recording of behavioral responses.

At the beginning of each trial, a central symbolic cue was presented for $100 \mathrm{~ms}$ prompting a covert shift of spatial attention to the cued location. At $600 \mathrm{~ms}$ after cue onset, the target stimulus was presented for $100 \mathrm{~ms}$ and participants had to indicate target detection with a button press within a $1200 \mathrm{~ms}$ time window. The trial was terminated once this time window had passed or immediately after the response was given. Trials were separated by a pseudo-randomized inter-trial interval of 800 ,

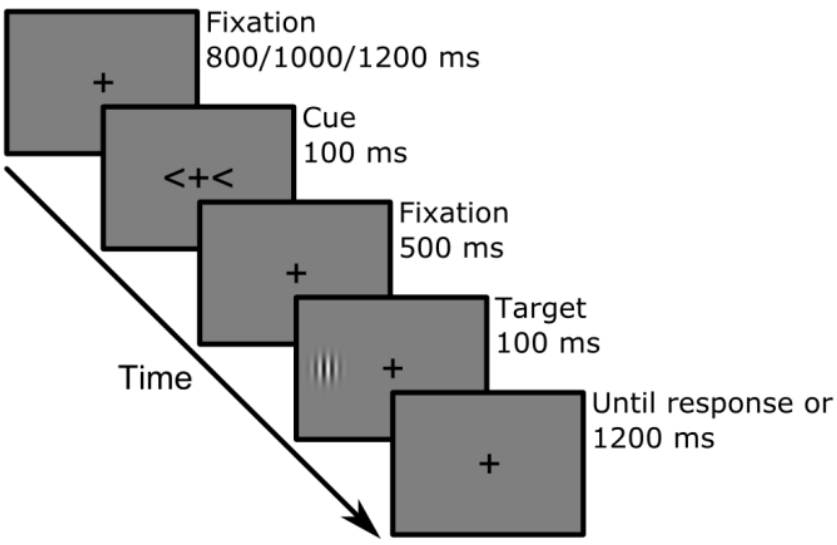

Figure 1. The sequence and timing of events for one possible trial is shown. In this case, the cue points to the left, prompting the participant to covertly shift attention to the left hemifield, and the target appears at the cued location (valid trial). A description of the other cue/target combinations is given in the main text. 
1000, or $1200 \mathrm{~ms}$. In case of false alarms, misses, anticipatory responses (RT below $100 \mathrm{~ms}$ or button press within the cue-target interval), or very slow responses (RT above $800 \mathrm{~ms}$ ), corrective feedback was given after the respective trial by showing an error message on the screen.

The combination of three cues (left, right, neutral) and three target locations (left, right, none/catch) resulted in nine conditions. Those trials with directional cues (left, right) provided information about where the target stimulus was most likely to appear ( $80 \%$ predictive validity). As a consequence, utilization of the cues was behaviorally relevant thereby encouraging participants to covertly shift spatial attention towards the cued location. In the majority of trials, the cues were valid, that is, they pointed towards the side of space where the target stimulus appeared but, occasionally, cues were invalid so that attention was shifted towards the opposite side of space. Neutral trials served as baseline condition where no spatial expectancy was induced but other factors such as perceptual processing, target detection, and behavioral response were identical to valid and invalid trials. On the behavioral level, reaction times on valid trials are typically faster compared to neutral trials (attentional benefits) and reaction times on invalid trials are typically slower compared to neutral trials (attentional costs). Conceptually, attentional benefits and attentional costs are commonly interpreted as reflecting two attentional processes, namely orienting of attention and reorienting of attention, respectively. This task has been specifically designed to isolate these processes and quantifies them as reaction time differences between neutral and valid/invalid trials.

\section{Magnetic Resonance Imaging}

Anatomical data were acquired for each participant to allow determination of individual stimulation sites based on Talairach coordinates (Talairach \& Tournoux, 1988). Imaging was performed with a Siemens Allegra 3.0 Tesla scanner equipped with a standard head coil (Siemens Medical Systems, Erlangen, Germany). High-resolution anatomical images covering the whole head were collected with a T1-weighted 3D ADNI MP-RAGE sequence (192 sagittal slices; matrix $=256 \times 256$; field of view $=256 \times 256 \mathrm{~mm}$; slice thickness $=1 \mathrm{~mm}$; no gap; 
in-plane voxel size $=1 \times 1 \mathrm{~mm}$; flip angle $=9^{\circ}$; repetition time $=2250 \mathrm{~ms}$; echo time $=2.6 \mathrm{~ms}$ ). Data were processed using the BrainVoyager QX 2.3 software package with default settings (Brain Innovation, Maastricht, The Netherlands). Anatomical data were corrected for intensity inhomogeneities and transformed to Talairach space (Talairach \& Tournoux, 1988). Then, a reconstruction of the cortical surface was created for both hemispheres to support TMS coil positioning by improving the visualization of the anatomical gyrification.

TMS target points were defined based on Talairach coordinates from the literature and projected on individual brain anatomy to guide TMS coil positioning (Figure 2). The coordinates for right IPS $(\mathrm{x}=23, \mathrm{y}=-65, \mathrm{z}=48)$ and left IPS $(x=-25, y=-63, z=48)$ were taken from a meta-analysis (He et al., 2007). These activation clusters were consistently found during spatial cueing tasks very similar to
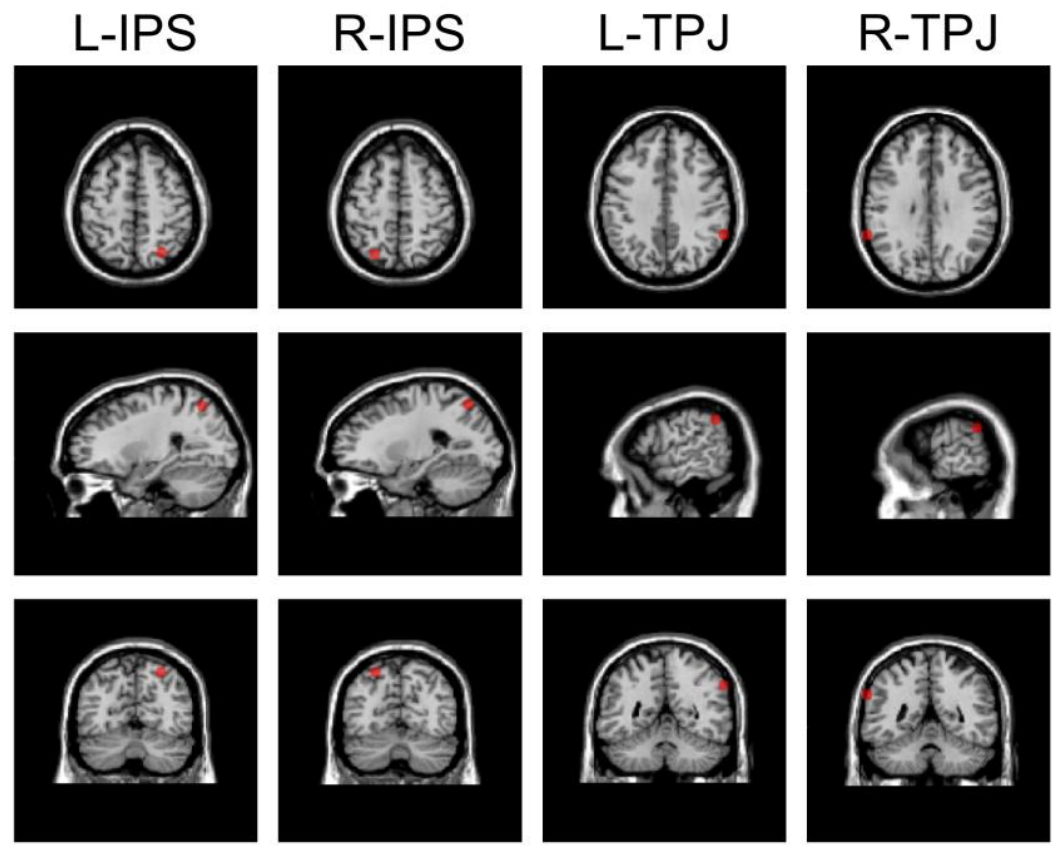

Figure 2. Anatomical location of Talairach coordinates for IPS and TPJ in both hemispheres. Images are in radiological convention and sagittal, coronal, and transverse sections are shown for each TMS target point. $\mathrm{R}=$ right hemisphere. $\mathrm{L}=$ left hemisphere. 
the one used in the present study. The coordinates for right TPJ $(x=60, y=-46$, $\mathrm{z}=28)$ and left TPJ $(\mathrm{x}=-56, \mathrm{y}=-48, \mathrm{z}=34)$ were taken from a single fMRI study that reported bilateral activation in the ventral fronto-parietal network, again, using a spatial cueing task very similar to ours (Doricchi et al., 2010). The vertex was defined as electrode position $\mathrm{Cz}$ according to the International 10-20 system (Jasper, 1958).

\section{Transcranial Magnetic Stimulation}

Transcranial magnetic stimulation was applied with a Medtronic MagPro X100 stimulator (Medtronic Functional Diagnostics A/S, Skovlunde, Denmark) and a figure-of-eight TMS coil (MC-B70; inner radius $=10 \mathrm{~mm}$; outer radius $=50 \mathrm{~mm}$ ). In order to ensure precise TMS coil positioning, we used the BrainVoyager TMS Neuronavigator system (BrainInnovation, Maastricht, The Netherlands) which allows real-time visualization of the TMS coil relative to individual anatomical data and predefined TMS target points. We navigated the TMS coil to the position where the distance between the center of the coil and the target point was as small as possible and the coil was manually held perpendicular to the skull. For all stimulation site, the coil was oriented parallel to the longitudinal fissure and the coil handle pointed posterior.

During each TMS session, we applied continuous theta burst stimulation for 40 seconds ( 600 pulses; $50 \mathrm{~Hz}$ triplets in a $5 \mathrm{~Hz}$ rhythm) over one of the five stimulation sites (right and left IPS, right and left TPJ, vertex) prior to task execution. In previous work, we successfully applied this protocol over frontal eye field to disrupt attentional processes (Duecker et al., 2013) and it has been shown to have long-lasting effects on cortical excitability for up to an hour (Huang, Edwards, Rounis, Bhatia, \& Rothwell, 2005). Stimulation intensity was set at $80 \%$ of the individual resting motor threshold (mean: $26.0 \%$ of maximum stimulator output; range $21 \%-32 \%$ ). The motor threshold was defined as the smallest intensity of a TMS pulse over right motor cortex that elicited a visible muscle twitch in the left 
index finger in three out of six trials. None of the participants reported any adverse side effects of the stimulation.

\section{Procedure and Design}

We conducted a pilot session prior to the TMS experiment in order to make sure that all participants performed that task as expected. The pilot session was identical to the TMS sessions with the exception that no TMS was applied. Participants were only included in the main experiment when they made use of the information provided by the cues and had no problems keeping central fixation during the trials. Specifically, we checked for statistically significant cueing effects and the presence of both attentional benefits and attentional costs. All participants passed this criterion and were invited for the main experiment.

All participants completed five TMS sessions based on a full within-subject design receiving TMS to right and left IPS, right and left TPJ, and vertex. TMS over vertex was expected to have no effects on task performance and was therefore considered a control condition. All TMS sessions were identical except for the stimulation site. The order of session was counterbalanced across participants and at least one day without TMS was required between TMS sessions in order to prevent potential carry-over effects. During TMS sessions, participants were comfortably seated in a chair with the head supported by a chin rest. After completion of 72 practice trials, TMS was applied to the brain area in question immediately followed by the spatial attention task. Within each session, participants completed 10 blocks consisting of 72 trials presented in randomized order. Short breaks between blocks were included and participants could rest as long as they wished before proceeding with the task. The overall duration of the behavioral task never exceeded 35 minutes. The proportion of valid:invalid:neutral:catch trials in each experimental block was 8:2:5:3, resulting in 320 valid trials, 80 invalid trials, 200 neutral trials, and 120 catch trials per TMS session. At the beginning of a block, four additional practice trials were included that were not considered in the analysis. Between blocks, participants received feedback about their average performance to ensure that they were motivated throughout the session. 


\section{Eye Movement Control}

Electrooculography (EOG) was used during task execution to allow offline control of fixation performance. This was essential because participants were required to covertly shift their attention (without moving their eyes) and eye blinks can interfere with the perception of the cue and target. Data were recorded bipolarly from two pairs of $\mathrm{Ag} / \mathrm{AgCl}$ electrodes with a BrainAmp ExG system (BrainProducts $\mathrm{GmbH}$, Munich, Germany). Electrodes were positioned at the outer canthus of each eye (horizontal EOG) and above and below the right eye (vertical EOG) in order to monitor eye movements and eye blinks. We kept the impedance of the electrodes below $5 \mathrm{k} \Omega$ and the signal was digitized at $1000 \mathrm{~Hz}$, high-pass filtered at $0.1 \mathrm{~Hz}$, and stored on disk using BrainVision Recorder (BrainProducts GmbH, Munich, Germany). Offline data analysis was performed with BrainVision Analyzer (BrainProducts $\mathrm{GmbH}$, Munich, Germany). Single trial data were visually inspected and all trials contaminated by eye movements or eye blinks between cue appearance and button press were excluded from further analysis.

\section{Statistical Analysis}

Trials were regarded as incorrect in case of false alarms, misses, anticipatory responses, or very slow responses and were excluded from analyses of reaction times. Additionally, trials following corrective feedback were discarded to allow reconcentration on the task. Similarly, trials following invalid trials were removed to counteract sequence effects which have been shown to negatively affect attentional benefits and costs due to strategic changes in the utilization of the cue (Jongen \& Smulders, 2007). Outliers were excluded according to the $1.5 \mathrm{x}$ IQR (interquartile range) criterion. After application of these exclusion criteria, $78 \%$ of all trials remained and were used to compute individual median reaction times. Across all conditions, the proportion of excluded trials never dropped below $75 \%$ and there were no systematic differences between conditions. On the individual level, median reaction times were always based on at least 20 trials per condition. Individual median reaction times were submitted to a three-way repeated-measures ANOVA 
with stimulation site (right IPS, left IPS, right TPJ, left TPJ, vertex), cue validity (valid, neutral, invalid), and hemifield (left, right) as within-subject factors. Not all levels of the factor stimulation site were included in each analysis as we examined the effects for the dorsal and ventral network separately. We also performed planned analyses of attentional benefits and costs by submitting the reaction time differences between neutral and valid/invalid trials to repeated-measures ANOVAS with stimulation site and hemifield as within-subject factors.

\section{Results}

\section{Errors and Eye Movements}

All participants performed the task with high levels of accuracy across all TMS sessions. On the individual level, accuracy never dropped below $90.0 \%$ indicating that participants were well able to detect the target stimuli and did not produce excessive numbers of false alarms due to failure to withhold the response on catch trials. On the group level, the mean accuracy across all TMS sessions was $97.7 \%$ with no significant differences between conditions as revealed by a repeatedmeasures ANOVA with stimulation site (right IPS, left IPS, right TPJ, left TPJ, vertex) as within-subject factor $(\mathrm{F}(4,72)=0.697, \mathrm{p}=.596)$. Similarly, the proportion of trials contaminated by eye movements was only $5.8 \%$ across all TMS sessions showing that participants had no difficulties in maintaining central fixation during trials. As above, a repeated-measures ANOVA confirmed that TMS had no stimulation site specific effect on eye movements $(\mathrm{F}(4,72)=0.763, \mathrm{p}=.553)$ showing that potential TMS effects on reaction times were not confounded or mediated by TMS-induced disruption of eye movement control. Finally, there was no evidence for speed-accuracy trade-offs so that the remaining analyses focused on TMS effects on reaction times (Table 1). 
Table 1. Reaction times (in milliseconds) and standard error of mean (in parentheses) for each experimental condition.

\begin{tabular}{|c|c|c|c|c|c|c|c|c|c|c|}
\hline \multirow[b]{2}{*}{ Cue } & \multicolumn{5}{|c|}{ Left hemifield } & \multicolumn{5}{|c|}{ Right hemifield } \\
\hline & $\begin{array}{l}\text { R- } \\
\text { IPS }\end{array}$ & $\begin{array}{c}\text { L- } \\
\text { IPS }\end{array}$ & $\begin{array}{l}\text { R- } \\
\text { TPJ }\end{array}$ & $\begin{array}{c}\text { L- } \\
\text { TPJ }\end{array}$ & Vertex & $\begin{array}{l}\text { R- } \\
\text { IPS }\end{array}$ & $\begin{array}{c}\text { L- } \\
\text { IPS }\end{array}$ & $\begin{array}{l}\mathrm{R}- \\
\mathrm{TPJ}\end{array}$ & $\begin{array}{c}\text { L- } \\
\text { TPJ }\end{array}$ & Vertex \\
\hline Valid & $\begin{array}{l}266 \\
(5.6)\end{array}$ & $\begin{array}{c}265 \\
(6.3)\end{array}$ & $\begin{array}{c}266 \\
(4.9)\end{array}$ & $\begin{array}{c}267 \\
(5.4)\end{array}$ & $\begin{array}{c}264 \\
(4.9)\end{array}$ & $\begin{array}{c}264 \\
(6.0)\end{array}$ & $\begin{array}{c}261 \\
(6.2)\end{array}$ & $\begin{array}{c}260 \\
(5.0)\end{array}$ & $\begin{array}{c}264 \\
(5.2)\end{array}$ & $\begin{array}{c}261 \\
(5.2)\end{array}$ \\
\hline Neutral & $\begin{array}{c}280 \\
(6.5)\end{array}$ & $\begin{array}{c}276 \\
(6.7)\end{array}$ & $\begin{array}{c}278 \\
(5.9)\end{array}$ & $\begin{array}{c}282 \\
(5.6)\end{array}$ & $\begin{array}{c}275 \\
(5.1)\end{array}$ & $\begin{array}{c}268 \\
(5.8)\end{array}$ & $\begin{array}{c}267 \\
(6.6)\end{array}$ & $\begin{array}{c}270 \\
(5.1)\end{array}$ & $\begin{array}{c}268 \\
(5.6)\end{array}$ & $\begin{array}{c}267 \\
(5.0)\end{array}$ \\
\hline Invalid & $\begin{array}{c}298 \\
(7.9)\end{array}$ & $\begin{array}{c}295 \\
(7.5)\end{array}$ & $\begin{array}{c}298 \\
(6.4)\end{array}$ & $\begin{array}{c}306 \\
(6.8)\end{array}$ & $\begin{array}{c}299 \\
(6.5)\end{array}$ & $\begin{array}{c}298 \\
(8.3)\end{array}$ & $\begin{array}{c}293 \\
(8.4)\end{array}$ & $\begin{array}{c}301 \\
(6.6)\end{array}$ & $\begin{array}{c}303 \\
(7.8)\end{array}$ & $\begin{array}{c}296 \\
(5.8)\end{array}$ \\
\hline Benefits & $\begin{array}{c}6.5 \\
(1.3)\end{array}$ & $\begin{array}{l}10.5 \\
(1.9)\end{array}$ & $\begin{array}{c}3.9 \\
(1.2)\end{array}$ & $\begin{array}{l}14.2 \\
(2.6)\end{array}$ & $\begin{array}{l}11.7 \\
(1.8)\end{array}$ & $\begin{array}{c}4.6 \\
(1.3)\end{array}$ & $\begin{array}{l}14.2 \\
(2.8)\end{array}$ & $\begin{array}{c}9.8 \\
(1.4)\end{array}$ & $\begin{array}{l}12.8 \\
(2.8)\end{array}$ & $\begin{array}{c}6.3 \\
(1.0)\end{array}$ \\
\hline Costs & $\begin{array}{l}26.2 \\
(4.8)\end{array}$ & $\begin{array}{c}18.9 \\
(3.7)\end{array}$ & $\begin{array}{l}35.3 \\
(4.8)\end{array}$ & $\begin{array}{l}24.5 \\
(4.0)\end{array}$ & $\begin{array}{l}23.6 \\
(4.4)\end{array}$ & $\begin{array}{l}29.8 \\
(5.0)\end{array}$ & $\begin{array}{l}18.4 \\
(4.2)\end{array}$ & $\begin{array}{l}30.4 \\
(4.4)\end{array}$ & $\begin{array}{l}19.6 \\
(3.3)\end{array}$ & $\begin{array}{l}29.0 \\
(3.3)\end{array}$ \\
\hline
\end{tabular}

\section{Task Performance under Baseline Conditions}

We first evaluated task performance after stimulation of the vertex (control site) in order to establish that the experimental procedure worked properly under baseline conditions. A repeated-measures ANOVA on median reaction times with cue (valid, neutral, invalid), and hemifield (right, left) as within-subject factors revealed significant main effects of cue $(\mathrm{F}(2,34)=61.146, \mathrm{p}<.001)$ and hemifield $(\mathrm{F}(1,17)=9.946, \mathrm{p}=.006)$ and a borderline significant interaction between cue and hemifield $(\mathrm{F}(2,34)=3.261, \mathrm{p}=.051)$. The main effect of cue resulted from the typical orienting effects observed in spatial cueing tasks (Figure 3) with valid trials producing faster and invalid trials producing slower reaction times compared to neutral trials ( $\mathrm{p}$-values for all pairwise comparisons $<.001$ ). This strongly indicates that participants made correct use of the cues by performing covert voluntary shifts of spatial attention towards the cued location. Importantly, the presence of these cueing effects allowed subsequent analyses in terms of attentional benefits and costs; a pre-requisite for answering several of our research questions. The main effect of 
hemifield resulted from generally faster reaction times for stimuli in the right hemifield most likely because participants had to respond with their right hand resulting in a spatial correspondence between response hand and hemifield, a phenomenon called Simon effect (Simon \& Rudell, 1967). The interaction between cue and hemifield (Figure 3) was due to higher attentional benefits in the left hemifield compared to the right hemifield $(\mathrm{t}(17)=3.014, \mathrm{p}=.008)$ and the opposite pattern was observed for attentional costs, that is, higher attentional costs in the right hemifield compared to the left hemifield $(\mathrm{t}(17)=2.166, \mathrm{p}=.045)$. This possibly indicates that shifting attention to the left hemifield is easier and reorienting attention away from the left hemifield is more difficult than the other way around. Alternatively, these differences could also be driven by an effect on neutral trials $(t(17)=4.489, p<.001)$ and it is difficult to decide between both alternatives based on the present data. Either way, the same observation has been reported before (Jongen, Smulders, \& Van Breukelen, 2006; Jongen \& Smulders, 2007) but an evidence-based explanation of this result is still lacking. Finally, the main effects and interaction described above were also found in subsequent analyses and the same explanations apply in those cases.

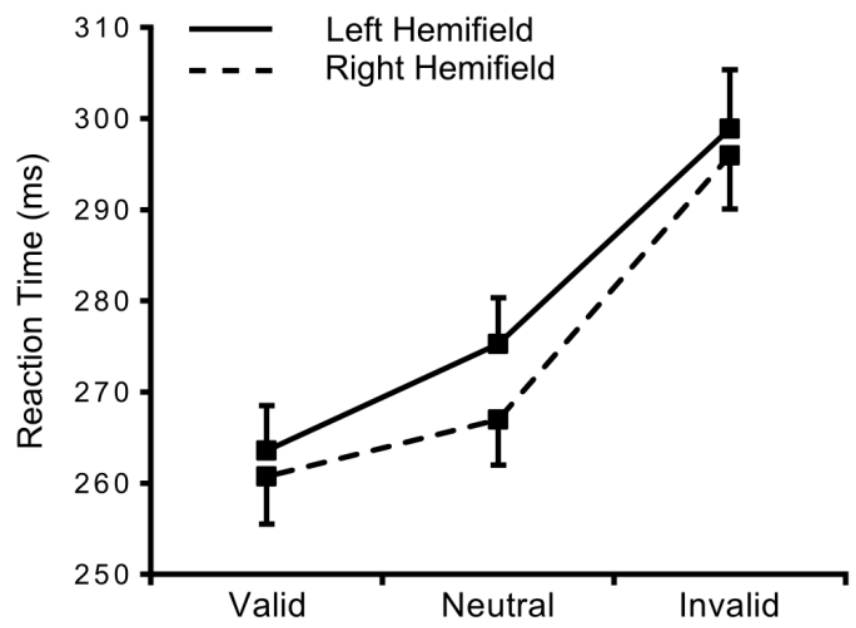

Figure 3. Main effect of cue after averaging reaction time data across the factors stimulation site and hemifield. The expected effect of cue validity was present with robust attentional benefits and costs. All pairwise comparisons are statistically significant at an alpha level of 0.001 and error bars depict standard errors. 


\section{Effect of TMS over the Dorsal Network on Reaction Times}

We then evaluated the effects of TMS over the dorsal fronto-parietal network in order to examine the functional asymmetry between right and left IPS. A repeatedmeasures ANOVA on median reaction times with stimulation site (right IPS, left IPS, vertex), cue (valid, neutral, invalid), and hemifield (right, left) as within-subject factors revealed significant main effects of cue $\left(F_{(2,34)}=57.779, p<.001\right)$ and hemifield $\left(F_{(1,17)}=6.042, p=.025\right)$ and a significant interaction between cue and hemifield $\left(F_{(2,34)}=5.229, p=.010\right)$. There was no main effect of stimulation site $\left(F_{(2,34)}=0.321, p=.728\right)$ and all interactions that included the factor stimulation site were far from being significant (all $p$-values $>.40$ ). It therefore appears that TMS had no influence on task performance and, as consequence, no functional asymmetry between right and left IPS was found. However, we nevertheless performed planned analyses to explore whether TMS had any effect on attentional benefits or costs to directly test our hypotheses that the dorsal network is involved in orienting and reorienting of attention. A repeated measures ANOVA on attentional benefits (Figure 4) with stimulation site (right IPS, left IPS, vertex) and hemifield (right, left)

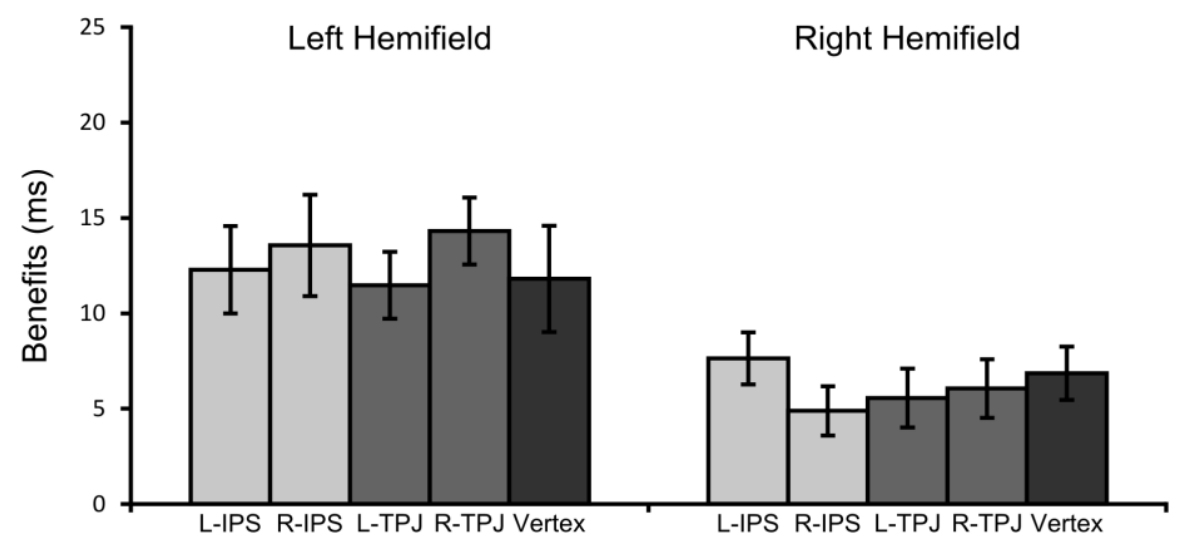

Figure 4. Attentional benefits for each hemifield after TMS over right and left IPS, right and left TPJ, and vertex. Attentional benefits are defined as the difference in reaction time between neutral and valid trials. $\mathrm{R}=$ right hemisphere. $\mathrm{L}=$ left hemisphere. Error bars depict standard errors. 
as within-subject factors confirmed that TMS did not systematically influence task performance with a non-significant main effect of stimulation site $\left(F_{(2,34)}=0.267\right.$, $p=.767)$ and no significant interaction between stimulation site and hemifield $\left(F_{(2,34)}=2.774, p=.077\right)$. Even though this interaction was relatively close to significance, follow-up exploration of simple effects did not reveal any differences between conditions that would allow speculations concerning the possible presence of true effects. Finally, the same analysis on attentional costs (Figure 5) also failed to reveal an effect of stimulation site with the main effect and interaction being far from significant (both $p$-values $>.40$ ). Obviously, these results neither support Heilman's "hemispatial" theory nor Kinsbourne's “opponent processor" model and the complete absence of any TMS effect in all conditions is contrary to wellestablished findings from previous work over parietal cortex. We therefore present additional observations in a later section that might account for the present results.

\section{Effect of TMS over the Ventral Network on Reaction Times}

We then evaluated the effects of TMS over the ventral fronto-parietal network in order to examine the functional asymmetry between right and left TPJ. A repeatedmeasures ANOVA on median reaction times with stimulation site (right TPJ, left TPJ, vertex), cue (valid, neutral, invalid), and hemifield (right, left) as within-subject factors revealed significant main effects of cue $(\mathrm{F}(2,34)=104.280, \mathrm{p}<.001)$ and hemifield $(\mathrm{F}(1,17)=7.515, \mathrm{p}=.014)$ and a significant interaction between cue and hemifield $(\mathrm{F}(2,34)=9.011, \mathrm{p}=.001)$. There was no main effect of stimulation site $(\mathrm{F}(2,34)=0.790, \mathrm{p}=.462)$ and all interactions that included the factor stimulation site were not significant (all p-values > .15). It therefore appears that TMS had no influence on task performance and, as consequence, no functional asymmetry between right and left TPJ was found. However, we nevertheless performed planned analyses to explore whether TMS had any effect on attentional benefits or costs to directly test our hypotheses that (1) right TPJ but not left TPJ is relevant for reorienting of attention (attentional costs) and (2) that a "virtual lesion" of the ventral network has an effect on functions associated with the dorsal network (attentional benefits). A repeated measures ANOVA on attentional benefits 
(Figure 4) with stimulation site (right TPJ, left TPJ, vertex) and hemifield (right, left) as within-subject factors confirmed that TMS did not systematically influence task performance but trends were nevertheless visible for the main effect of stimulation site $(\mathrm{F}(2,34)=2.526, \mathrm{p}=.095)$ and the interaction between stimulation site and hemifield $(\mathrm{F}(2,34)=3.220, \mathrm{p}=.052)$. However, follow-up exploration of simple effects did not reveal differences with respect to vertex stimulation and the most robust effect was a reduction of attentional benefits in the right hemifield after left TPJ stimulation, a finding that is contrary to current models of attention control and certainly lacks force in the light of a null result for right TPJ stimulation. Finally, the same analysis on attentional costs (Figure 5) also failed to reveal an effect of stimulation site with the main effect and interaction being far from significant (both p-values > .40). In sum, these results fail to shed new light on the functional asymmetry between right and left TPJ and do not support the hypothesis that a "virtual lesion" to the ventral network also affects functions of the dorsal network. Just as in the previous section, the complete absence of any TMS effect in all conditions is somewhat surprising increasing the need to explore potential factors that might have contributed to these null results.

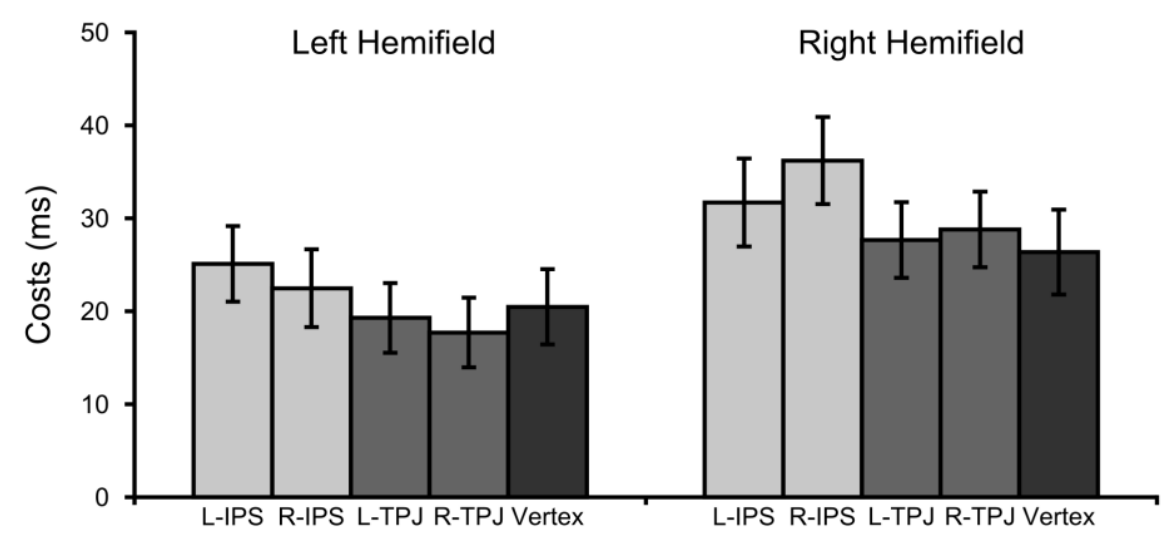

Figure 5. Attentional costs for each hemifield after TMS over right and left IPS, right and left TPJ, and vertex. Attentional benefits are defined as the difference in reaction time between neutral and invalid trials. $\mathrm{R}=$ right hemisphere $\mathrm{L}=$ left hemisphere. Error bars depict standard errors. 


\section{Exploration of Sources of Variability}

One possible explanation for the absence of TMS effects on task performance are sequence effects across TMS sessions that overshadow any modulation of attentional processes. We therefore analyzed the data to explore the effect of session on attentional benefits and costs in order to evaluate the effectiveness of the task to elicit shifts of spatial attention over time. We collapsed the data across stimulation sites and hemifields and then performed repeated-measures ANOVAs on attentional benefits and costs with session (one, two, three, four, five) as within-subject factor. This analyses did not reveal changes of attentional benefits $(\mathrm{F}(4,68)=0.606, \mathrm{p}=$ $.659)$ and costs $(\mathrm{F}(4,68)=1.402, \mathrm{p}=.243)$ over sessions indicating that cueing was equally effective throughout the experiment on the group level. However, attentional benefits were rather small on average $(\mathrm{M}=9.5 \mathrm{~ms})$ suggesting that cues were less effective than in our previous study (Duecker et al., 2013) where attentional benefits were considerably higher ( $\mathrm{M}=15 \mathrm{~ms}$ ), even with inclusion of TMS sessions that reduced attentional benefits. Moreover, attentional benefits in the pilot session $(\mathrm{M}=$ $18.1 \mathrm{~ms})$ were much higher than in all subsequent TMS sessions ( $\mathrm{M}=9.5 \mathrm{~ms})$ suggesting that participants did not take as much advantage of the cues as in the first session. Regardless of the underlying reasons, relatively small attentional benefits pose a problem because they essentially reduce the room for modulations by TMS because negative attentional benefits are highly unlikely as this would indicate that advance information about the location of a target stimulus leads to worse performance than providing no information at all.

Even though attentional benefits and costs were very stable over time on the group level, there might still be considerable variability within subjects over sessions which can also pose a problem. We therefore calculated the range of values within each hemifield across sessions for each participant as a measure of variability. This revealed that attentional benefits and costs differed between session, on average, by $19.3 \mathrm{~ms}$ and $39.6 \mathrm{~ms}$, respectively. This degree of variability is certainly problematic and indicates that participants were unable to perform equally well across sessions. 


\section{Discussion}

There are three competing theories of attentional control that are capable of explaining the pre-dominance of spatial neglect after right hemisphere damage. Each of them proposes hemispheric asymmetries either within the dorsal or ventral frontoparietal network. Here, we used TMS to create "virtual lesions" in core nodes of both networks and assessed the behavioral consequences of this disruption on a spatial cueing task in order to shed new light on the functional mechanism underlying attention control, thereby putting current functional-anatomical models to the test. Specifically, we addressed (1) the hemispheric asymmetry within the dorsal network between right and left IPS, (2) the right-lateralization of the ventral network (TPJ), and (3) the consequences of disrupting the ventral network on attentional functions associated with the dorsal network. Unfortunately, we did not observe any effect of TMS on task performance making it impossible to draw any novel conclusions regarding the functional roles of the dorsal and ventral network. Given the abundance of TMS and neuroimaging studies that support the involvement of the dorsal and ventral network in attentional control, it is practically impossible that the present null result could be seen as evidence that the stimulated brain areas are not functionally relevant for shifts of spatial attention as measured with our task. In the following, we therefore discuss possible reasons for this null result.

One of the reasons why we were unable to reveal any effect of TMS on task performance is probably related to the high within-subject variability across sessions. We used a full within-subject design, meaning that each participant had to perform the same task during five sessions, one for each stimulation site. An important prerequisite therefore is that task performance is rather stable across sessions because any effect due to TMS might otherwise be overshadowed by other non-specific factors that influence task performance on any given day. As shown in the results section, performance was stable across sessions on the group level but the within-subject variability was much higher than the modulation expected due to TMS. This had a huge negative influence on statistical power which we did not counteract by increasing the number of participants. The underlying reasons for this high within-subject variability are difficult to identify but the experimenter noticed 
that motivational factors were particularly variable across sessions. As one might expect, participants were not very keen on doing the same task over and over again potentially influencing participant's effort and compliance with task demands. For that reason, future efforts to investigate our research questions might benefit from addressing each of them separately thereby reducing the number of sessions hopefully leading to more consistent task performance.

A critical prerequisite for finding an effect of TMS on task performance is accurate TMS coil positioning. If TMS is not applied to the functional brain area of interest it is obviously impossible to induce the intended neural effect and, as a consequence, task performance will most likely remain unaffected. In our previous study over frontal cortex (Duecker et al., 2013) TMS target points were defined based on individual activation foci as measured with fMRI for each participant. This procedure is widely considered the gold standard that maximizes effects sizes and minimizes the number of participants required to reach significance (Sack et al., 2009). Nevertheless, we did not obtain functional data for each participant in the present study but defined TMS target points based on Talairach coordinates from the literature instead. The primary motivation for this methodological difference was difficulties in localizing IPS and TPJ on an individual basis. Presumably for the same reason, previous TMS studies have also opted for suboptimal localization methods and defined TMS target points based on the International 10-20 EEG system (Dambeck et al., 2006; Hilgetag et al., 2001), brain anatomy (Silvanto et al., 2009), or Talairach (functional group) coordinates (Chica, Bartolomeo, \& ValeroCabré, 2011). Given that previous work has successfully applied TMS even with the very same Talairach coordinates that were used here (Chica et al., 2011), we trusted that localization based on functional group data would be sufficient despite methodological differences between studies; we here used an offline "virtual lesion" approach whereas Chica et al. applied TMS pulses during task execution. Having said this, the accuracy of Talairach coordinates always depends on the specific sample and it is safe to assume that this sub-optimal localization approach contributed to the present null result. On the upside, the present study has contributed to raise awareness of the limitations of TMS coil positioning based on Talairach coordinates and has inspired the work presented in Chapter 4. 
In summary, the present study failed to explore hemispheric asymmetries in the dorsal and ventral fronto-parietal network due to statistical and methodological reasons. However, the research questions addressed here remain of high relevance for current models of attention control and the reasons for the pre-dominance of spatial neglect after right hemisphere damage remain a relevant and timely research question. Despite the problems encountered in the present study, previous work demonstrates that TMS can play an important role in revealing the functional roles of and functional asymmetries between brain areas.

\section{Acknowledgements}

This work was supported by the European Research Council under the European Union's Seventh Framework Programme (FP7/2007-2013) / ERC Grant agreement (263472 to A.T.S.), and the Netherlands Organization for Scientific Research (40007-230 to A.T.S \& E.F.). We thank our medical supervisor Cees van Leeuwen and independent physician Martin van Boxtel. 


\section{References}

Chica, A. B., Bartolomeo, P., \& Valero-Cabré, A. (2011). Dorsal and Ventral Parietal Contributions to Spatial Orienting in the Human Brain. The Journal of Neuroscience, 31(22), 8143-8149.

Corbetta, M., Kincade, M. J., Lewis, C., Snyder, A. Z., \& Sapir, A. (2005). Neural basis and recovery of spatial attention deficits in spatial neglect. Nature Neuroscience, 8(11), 1603-1610.

Corbetta, M., \& Shulman, G. L. (2002). Control of goal-directed and stimulusdriven attention in the brain. Nature Reviews Neuroscience, 3(3), 201-215.

Corbetta, M., \& Shulman, G. L. (2011). Spatial neglect and attention networks. Annual Review of Neuroscience, 34(1), 569-599.

Dambeck, N., Sparing, R., Meister, I. G., Wienemann, M., Weidemann, J., Topper, R., \& Boroojerdi, B. (2006). Interhemispheric imbalance during visuospatial attention investigated by unilateral and bilateral TMS over human parietal cortices. Brain Research, 1072(1), 194-199.

Doricchi, F., Macci, E., Silvetti, M., \& Macaluso, E. (2010). Neural correlates of the spatial and expectancy components of endogenous and stimulus-driven orienting of attention in the Posner task. Cerebral Cortex, 20(7), 1574-1585.

Duecker, F., Formisano, E., \& Sack, A. T. (2013). Hemispheric Differences in the Voluntary Control of Spatial Attention: Direct Evidence for a Right-Hemispheric Dominance within Frontal Cortex. Journal of Cognitive Neuroscience.

Grosbras, M.-H., \& Paus, T. (2003). Transcranial magnetic stimulation of the human frontal eye field facilitates visual awareness. European Journal of Neuroscience, 18, 3121-3126. 
He, B. J., Snyder, A. Z., Vincent, J. L., Epstein, A., Shulman, G. L., \& Corbetta, M. (2007). Breakdown of functional connectivity in frontoparietal networks underlies behavioral deficits in spatial neglect. Neuron, 53(6), 905-918.

Heilman, K. M., \& Abell, T. Van Den. (1980). Right hemisphere dominance for attention: The mechanism underlying hemispheric asymmetries of inattention (neglect). Neurology, 30(3), 327-330.

Hilgetag, C. C., Theoret, H., \& Pascual-Leone, A. (2001). Enhanced visual spatial attention ipsilateral to rTMS-induced "virtual lesions" of human parietal cortex. Nature Neuroscience, 4(9), 953-957.

Hopfinger, J. B., Buonocore, M. H., \& Mangun, G. R. (2000). The neural mechanisms of top-down attentional control. Nature Neuroscience, 3(3), 284-291.

Huang, Y.-Z., Edwards, M. J., Rounis, E., Bhatia, K. P., \& Rothwell, J. C. (2005). Theta burst stimulation of the human motor cortex. Neuron, 45(2), 201-206.

Jasper, H. H. (1958). The ten-twenty electrode system of the International Federation. Electroencephalography and Clinical Neurophysiology, 10, 371-375.

Jongen, E. M. M., \& Smulders, F. T. Y. (2007). Sequence effects in a spatial cueing task: endogenous orienting is sensitive to orienting in the preceding trial. Psychological Research, 71(5), 516-523.

Jongen, E. M. M., Smulders, F. T. Y., \& Van Breukelen, G. J. P. (2006). Varieties of attention in neutral trials: Linking RT to ERPs and EEG frequencies. Psychophysiology, 43(1), 113-125.

Kastner, S., Pinsk, M. A., De Weerd, P., Desimone, R., \& Ungerleider, L. G. (1999). Increased activity in human visual cortex during directed attention in the absence of visual stimulation. Neuron, 22(4), 751-761.

Kincade, J. M., Abrams, R. A., Astafiev, S. V, Shulman, G. L., \& Corbetta, M. (2005). An event-related functional magnetic resonance imaging study of voluntary 
and stimulus-driven orienting of attention. The Journal of Neuroscience, 25(18), 4593-4604.

Kinsbourne, M. (1977). Hemi-neglect and hemisphere rivalry. Advances in Neurology, 18, 41-49.

Mesulam, M. M. (1981). A cortical network for directed attention and unilateral neglect. Annals of Neurology, 10(4), 309-325.

Posner, M. I. (1980). Orienting of attention. Quarterly Journal of Experimental Psychology, 32(1), 3-25.

Posner, M. I., Snyder, C. R., \& Davdison, B. J. (1980). Attention and the detection of signals. Journal of Experimental Psychology, 109(2), 160-174.

Sack, A. T., Cohen Kadosh, R., Schuhmann, T., Moerel, M., Walsh, V., \& Goebel, R. (2009). Optimizing functional accuracy of TMS in cognitive studies: a comparison of methods. Journal of Cognitive Neuroscience, 21(2), 207-221.

Seyal, M., Ro, T., \& Rafal, R. (1995). Increased sensitivity to ipsilateral cutaneous stimuli following transcranial magnetic stimulation of the parietal lobe. Annals of Neurology, 38(2), 264-267.

Shulman, G. L., Pope, D. L. W., Astafiev, S. V, McAvoy, M. P., Snyder, A. Z., \& Corbetta, M. (2010). Right hemisphere dominance during spatial selective attention and target detection occurs outside the dorsal frontoparietal network. The Journal of Neuroscience, 30(10), 3640-3651.

Silvanto, J., Lavie, N., \& Walsh, V. (2006). Stimulation of the human frontal eye fields modulates sensitivity of extrastriate visual cortex. Journal of Neurophysiology, 96(2), 941-945.

Silvanto, J., Muggleton, N., Lavie, N., \& Walsh, V. (2009). The Perceptual and Functional Consequences of Parietal Top-Down Modulation on the Visual Cortex. Cerebral Cortex, 19(2), 327-330. 
Simon, J. R., \& Rudell, A. P. (1967). Auditory S-R compatibility: The effect of an irrelevant cue on information processing. Journal of Applied Psychology, 51(3), 300-304.

Szczepanski, S. M., Konen, C. S., \& Kastner, S. (2010). Mechanisms of spatial attention control in frontal and parietal cortex. The Journal of Neuroscience, 30(1), $148-160$.

Talairach, J., \& Tournoux, P. (1988). Co-Planar Stereotaxic Atlas of the Human Brain. New York: Thieme. 


\section{Chapter 4}

\section{The Cortex-Based Alignment Approach to TMS Coil Positioning}

Based on:

Duecker, F., Frost, M. A., de Graaf, T. A., Graewe, B., Jacobs, C., Goebel, R., Sack, A. T. (under review). The Cortex-Based Alignment Approach to TMS Coil Positioning. 


\section{Abstract}

Transcranial magnetic stimulation (TMS) allows non-invasive manipulation of brain activity in humans. The effectiveness of TMS experiments critically depends on precise TMS coil positioning, which is best for most brain areas when a frameless stereotactic system is used to target activation foci based on individual functional magnetic resonance imaging (fMRI) data. From a purely scientific perspective, individual fMRI-guided TMS is thus the method of choice to ensure optimal TMS efficiency. Yet, from a more practical perspective, such individual functional data are not always available, and therefore alternative TMS coil positioning approaches are often applied, e.g., based on functional group data reported in Talairach coordinates. We here propose a novel method for TMS coil positioning that is based on functional group data, yet only requires individual anatomical data. We used cortex-based alignment (CBA) to transform individual anatomical data to an atlas brain that includes probabilistic group maps of two functional regions (FEF and V5/hMT+). Then, these functional group maps were back-transformed to the individual brain anatomy, preserving functional-anatomical correspondence. As a proof of principle, the resulting CBA-based functional targets in individual brain space were compared with individual FEF and V5/hMT+ hotspots as conventionally localized with individual fMRI data, and with targets based on Talairach coordinates as commonly done in TMS research in case only individual anatomical data are available. Our CBA-based approach significantly improved localization of functional brain areas compared to traditional Talairach-based targeting. Given the widespread availability of cortex-based alignment schemes and pre-existing functional group data, the proposed procedure is easy to implement and at no additional measurement costs. However, the accuracy of individual fMRI-guided TMS remains unparalleled and the CBA-based approach should only be the method of choice when individual functional data cannot be obtained or experimental factors argue against it. 


\section{Introduction}

Transcranial magnetic stimulation (TMS) is a non-invasive brain interference technique that is widely used to investigate brain-behavior relationships in the healthy and diseased human brain (Hallett, 2000; Pascual-Leone, Walsh, \& Rothwell, 2000; Sack, 2006 ; Walsh \& Cowey, 2000). TMS can provide excellent temporal resolution, due to the brevity of a single TMS pulse (less than one millisecond), and good spatial resolution. The spatial resolution of TMS critically depends on the TMS coil design (Deng, Lisanby, \& Peterchev, 2012). In the majority of studies, so-called figure-of-eight coils are used that are purpose-built to generate a very focal magnetic field and thus allow selective stimulation of individual functional brain areas. This spatial specificity of TMS is very desirable in most cases and is paramount for investigating "causal" structure-function relationships. However, effective spatial resolution depends not only on coil-design, but also on the accuracy of TMS coil positioning. In fact, the more spatially specific TMS is, the more important it becomes to ensure that TMS is applied exactly to the brain location of interest.

Different approaches for TMS coil positioning have been developed over the years (Sack et al., 2009; Sparing, Buelte, Meister, Paus, \& Fink, 2008). In some cases, the immediate consequences of a single TMS pulse can be used as an index for effective stimulation. By moving along primary motor cortex one can induce muscle twitches that correspond to the organization of the motor "homunculus" allowing identification of the optimal TMS coil position to target the intended motor representation (e.g. Wassermann et al., 1992). Similarly, TMS over early visual cortex induces phosphenes that correspond to the retinotopic organization of visual cortex (e.g. Kammer et al., 2005). When the TMS coil is positioned based on such functional markers, the resulting TMS target points have been shown to closely match localization based on individual fMRI data and may be considered optimal (Salminen-Vaparanta, Noreika, Revonsuo, Koivisto, \& Vanni, 2012; Thielscher, Reichenbach, Uğurbil, \& Uludağ, 2010; Thompson, Aaen-Stockdale, Koski, \& Hess, 2009). However, the majority of brain areas are "silent", i.e., a TMS pulse does not produce a readily observable response thus requiring alternative TMS coil 
positioning approaches. In early work, the TMS coil was positioned relative to functional markers as described above or simply shifted several centimeters from cranial landmarks like the inion. Another popular method was to borrow the international 10-20 coordinate system from EEG research (Jasper, 1958), which assumes that certain electrode positions correspond to certain anatomical regions (see Herwig, Satrapi, \& Schönfeldt-Lecuona, 2003). But as the availability of imaging tools increased, 'neuronavigation' by a frameless stereotactic system became a preferred method overcoming various limitations of previous approaches. Both the TMS coil and the subject's head are tracked and co-registered in space, allowing precise (online) targeting of a pre-defined brain area (Cohen Kadosh et al., 2007; Herwig et al., 2002; Paus, 1999; Sack et al., 2006; Schönfeldt-Lecuona et al., 2005).

This brain area can be defined in several ways. First, one might determine the TMS 'target point' based on individual anatomical data. For instance, one could inspect the anatomical data and manually define the inferior parietal sulcus. For many brain areas, this approach is not necessarily accurate because the macroanatomical organization of the brain does not always predict where a functional brain area is located (Frost \& Goebel, 2012). Moreover, this approach requires expertise to correctly identify anatomical landmarks and is subjective; thus prone to human error. A second, commonly used, approach is to base TMS target points on coordinates in a common reference space, such as MNI or Talairach space (Talairach \& Tournoux, 1988). These coordinates are generally based on fMRI group data from prior experiments or taken from the literature. This procedure only requires individual anatomical data, which are transformed into the same reference space in order to project the Talairach coordinates on them. However, this approach is limited, since Talairach coordinates are represented in volume space, the macroanatomical variability across subjects is completely ignored, and the extent of functional-anatomical correspondence of brain areas is not taken into account (Frost $\&$ Goebel, 2012). A third approach makes full use of neuroimaging data by utilizing both individual anatomical and functional data. The target area is functionally defined in each individual subject in a preceding fMRI experiment, and targeted directly with neuronavigation. As the research question for the TMS experiment is in 
most cases whether or not the observed functional activation involves a functionally relevant process, this localization scheme seems most appropriate.

We recently empirically evaluated the impact of these various localization schemes on behavioral effect sizes and statistical power (Sack et al., 2009; also see Sparing et al., 2008). The inferior parietal sulcus was determined by aforementioned localization schemes, and the effects of TMS on task performance were evaluated for each resulting TMS site. We showed that the number of participants required for a TMS effect to reach significance can increase dramatically when localization accuracy is suboptimal. As expected, individual functional localization of TMS target points based on individual fMRI data outperformed all other available methods. Specifically, TMS effects were significant with just five participants using fMRI-based neuronavigation, whereas neuronavigation based on Talairach coordinates reached the same significance level only after 13 participants.

In summary, localization methods that do not utilize individual functional data for TMS target point localization come at a cost that can be of considerable practical relevance. As shown above, the choice to use individual fMRI-guided TMS can be decisive for finding a significant TMS effect. Yet, the individual functional localization method may not always be feasible or acceptably cost-efficient. While appropriate functional data may generally be lacking for the recruited subject sample, previously acquired anatomical data is much more widely available. In these cases, traditionally the Talairach- (or MNI-) based approach has been applied as it only requires such anatomical data prior to TMS. However, the TAL- or MNIapproach have various shortcomings and demonstrably limited power, as discussed above. Here, we propose a novel method for TMS coil positioning that overcomes some of these shortcomings while still only requiring anatomical data for each subject as well as pre-existing functional group data. As will be explained below, we refer to this as the "CBA-based approach" for localization.

In recent years advanced whole-brain alignment schemes have been developed that exploit curvature information of the cortical surface to minimize macro-anatomical variability across subjects, in order to increase functional overlap on the group level (Fischl, Sereno, \& Dale, 1999; Fischl, Sereno, Tootell, \& Dale, 1999; Frost \& 
Goebel, 2012; Goebel, Esposito, \& Formisano, 2006). We suggest that these methods can also be used to improve TMS targeting in the absence of individual functional data. To demonstrate, we here use cortex-based alignment (CBA) to transform individual anatomical data to an atlas brain that includes functional probabilistic group maps of the FEF and V5/hMT+. Then, these functional group maps are back-transformed to the individual brain anatomy, preserving functionalanatomical correspondence. These atlas-based functional hotspots can thus serve as TMS target points. As a proof of principle, we measured individual FEF and $\mathrm{V} 5 / \mathrm{hMT}+$ hotspots (serving as 'baseline') with standard localizers, to evaluate the relative locations of TMS target sites based on 1) these individual localizers, 2) our CBA-based approach, and 3) Talairach coordinates. Importantly, this approach can be generalized to any existing fMRI data set in surface space, allowing the use of previously obtained functional results instead of publicly available probabilistic atlases if desired. Given the widespread availability of cortex-based alignment schemes and pre-existing functional group data, the proposed procedure is easy to implement and at no additional measurement costs compared to traditional Talairach- or MNI-based targeting.

\section{Methods and Materials}

\section{Participants}

Twelve participants (6 female; aged 25-31) were recruited from the Maastricht University community. All had normal or corrected-to-normal vision, no history of neurological or psychiatric illness, and were screened for fMRI experimentation safety. Written informed consent was obtained prior to participation and the study was approved by the ethical committee of the Faculty of Psychology and Neuroscience at Maastricht University. 


\section{Functional Magnetic Resonance Imaging}

Anatomical and functional data were acquired with a Siemens Allegra 3.0 Tesla scanner equipped with a standard head coil (Siemens Medical Systems, Erlangen, Germany). High-resolution anatomical images covering the whole head were collected with a T1-weighted 3D ADNI MP-RAGE sequence (192 sagittal slices; matrix $=256 \times 256$; field of view $=256 \times 256 \mathrm{~mm}$; slice thickness $=1 \mathrm{~mm}$; no gap; in-plane voxel size $=1 \times 1 \mathrm{~mm}$; flip angle $=90^{\circ}$; repetition time $=2250 \mathrm{~ms}$; echo time $=2.6 \mathrm{~ms}$ ). Functional images were obtained using a $\mathrm{T} 2 *$-weighted single shot echo-planar imaging (EPI) sequence (32 oblique slices with full-brain coverage; matrix $=64 \times 64$; field of view $=224 \times 224 \mathrm{~mm}$; slice thickness $=3.5 \mathrm{~mm}$; no gap; in-plane voxel size $=3.5 \times 3.5 \mathrm{~mm}$; flip angle $=90^{\circ}$; repetition time $=2000 \mathrm{~ms}$; echo time $=30 \mathrm{~ms}$ ). Visual stimuli were back-projected on a screen at the rear end of the scanner bore and could be seen by the participants via a mirror system attached to the head coil.

\section{Functional Localizers}

We used standard functional localizers in order to determine the position of FEF and hMT/V5+ in each participant (procedures identical to Frost \& Goebel, 2012). For FEF localization, a block design was used with alternating blocks of central fixation and saccadic eye movements. During saccadic eye movement blocks, participants had to follow a circle with their eyes that appeared at one out of eight pre-defined locations along the horizontal and vertical meridian at a rate of $2 \mathrm{~Hz}$. In total, participants completed 21 blocks each lasting 16 seconds. The contrast used to identify FEF was "saccades > fixation". For the hMT/V5+ localizer, blocks with a central fixation point were alternated with blocks of stationary or moving dots that were passively viewed. Dot displays consisted of 400 dots that either moved radially outwards from the center of the screen or remained stationary throughout the block. Participants were instructed to keep central fixation at all times. In total, participants completed 17 blocks each lasting 16 seconds. The contrast used to identify hMT/V5+ was "moving dots > stationary dots". 


\section{Retrieving FEF and hMT/V5+ TMS Target Points from Independent Group Data}

The central aim of the present study was to assess localization precision of TMS target points in cases where individual functional localizers are unavailable (Figure 1). This is conventionally based on coordinates in a standard system, such as Talairach space. To test the efficacy of our CBA-based approach (see below), we used a second, and independent data set from a previous study (Frost \& Goebel, 2012) to estimate the position of FEF and hMT/V5+ from these group data $(\mathrm{N}=10)$ in each participant. In contrast to most practical situations in TMS research, the localization procedures were actually identical to the ones used for the present study (outlined above) in order to prevent any confounding effects due to task differences. The later comparison between localization methods would therefore be as 'clean' as possible. These group data were available in 'volume space' where Talairach coordinates defined the location of FEF and hMT/V5+ as they are commonly obtained with a univariate analysis and in 'surface space' where probabilistic functional maps indicated the area of highest probability for each cluster (for details see Frost \& Goebel, 2012). The data represented in surface space were used for our CBA-based approach.

For the Talairach-based approach, we projected the independent group Talairach coordinates back to the individual brain anatomy of the participants from the present

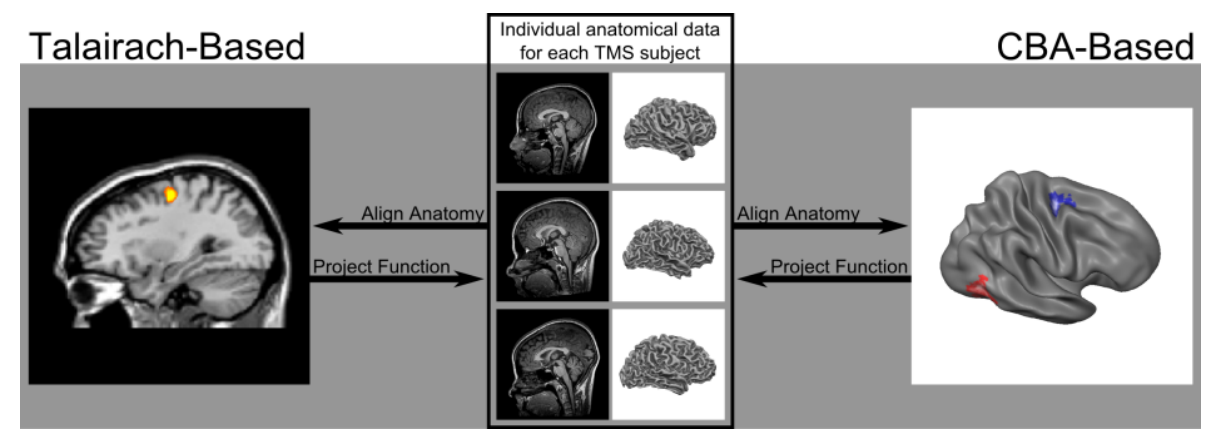

Figure 1. Schematic representation of the traditional Talairach-based approach and our CBA-based approach. Individual anatomical data are aligned to an atlas/reference brain either in volume space based on a few anatomical landmarks, as shown on the left, or in surface space based on the macro-anatomical structure of the brain, as shown on the right. Once this alignment is performed, existing functional data can be back-projected to individual space by applying the inverse transformation. 
study. For the CBA-based approach, we used the individual anatomical data to create a reconstruction of the cortical surface. Individual anatomical data were then aligned to the average group brain of the independent data set described above using CBA. This alignment process first extracts curvature information from the cortical mesh reconstructions which are then inflated to a sphere. CBA then aligns in a "coarse to fine" fashion, first aligning heavily smoothed maps which only contain the most prominent macro-anatomical landmarks (the central sulcus, the Sylvian Fissure, the superior temporal sulcus etc) in order to avoid gross misalignment. The next level of alignment uses less smoothed curvature maps and so on until the final stages where alignment proceeds on unsmoothed maps which contain all anatomical details. CBA calculates gradient information from curvature maps and utilizes these gradients to iteratively reduce misalignment, through local vertex movement, to a target brain, which here was a previously published atlas brain (see Frost and Goebel, 2012). Finally, using the inverse of this transformation, the probabilistic functional atlas was back-transformed to the individual brain anatomy where the center of gravity for the area with the highest probability of each cluster (FEF and hMT/V5+) was defined as TMS target point.

\section{Data Analysis}

Following the procedures described above, we obtained three sets of coordinates for each brain area (FEF and hMT/V5+) and participant, namely 1) the individual hotspot based on functional localization, 2) a Talairach-based hotspot obtained from the independent group data set in volume space, and 3) a CBA-based hotspot obtained from the same independent group data set in surface space. The precision of the different localization approaches was then quantified in terms of Euclidean distances between the individually defined hotspots and the Talairach-based and CBA-based hotspots. This measure of distance between hotspots was then used for further statistical analysis.

The performance of Talairach-based and CBA-based targeting was tested with repeated-measures analysis of variance (ANOVA) with brain area (FEF and hMT/V5+) and localization approach (CBA and Talairach) as within subject factors. 
Additionally, we hypothesized that CBA-based targeting is more accurate than localization based on Talairach coordinates, and therefore compared both approaches for each brain area directly with one-sided paired $t$-tests.

Table 1. Talairach coordinates of individual functional hotspots of FEF and hMT/V5+ for each participant. All values are provided in millimeters. SEM = standard error of the mean. Talairach conventions: $\mathrm{x}=$ left to right, $\mathrm{y}=$ back to front, $\mathrm{z}=$ bottom to top.

\begin{tabular}{|c|c|c|c|c|c|c|}
\hline \multirow[b]{2}{*}{ Participant } & \multicolumn{3}{|c|}{ FEF } & \multicolumn{2}{|c|}{ hMT/V5+ } & \multirow[b]{2}{*}{$z$} \\
\hline & $x$ & $y$ & $z$ & $x$ & $y$ & \\
\hline 1 & 25 & -8 & 46 & 41 & -72 & 5 \\
\hline 2 & 26 & -10 & 58 & 46 & -68 & -2 \\
\hline 3 & 29 & -14 & 56 & 49 & -58 & -6 \\
\hline 4 & 22 & 0 & 54 & 40 & -61 & 5 \\
\hline 5 & 36 & -15 & 55 & 42 & -68 & 3 \\
\hline 6 & 22 & -8 & 53 & 47 & -65 & 4 \\
\hline 7 & 26 & -10 & 51 & 51 & -58 & -2 \\
\hline 8 & 25 & -13 & 46 & 42 & -67 & 2 \\
\hline 9 & 26 & -20 & 53 & 42 & -85 & -7 \\
\hline 10 & 20 & -9 & 54 & 33 & -73 & -4 \\
\hline 11 & 26 & -14 & 53 & 39 & -72 & -4 \\
\hline 12 & 30 & -13 & 44 & 49 & -64 & -8 \\
\hline Mean & 26 & -11 & 52 & 43 & -68 & -1 \\
\hline SEM & 0.4 & 0.4 & 0.4 & 0.4 & 0.6 & 0.4 \\
\hline Range & 16 & 20 & 14 & 18 & 27 & 13 \\
\hline
\end{tabular}




\section{Results}

To evaluate our CBA-based approach, we applied several methods of target localization. Given that individual functional localizers are the gold standard both on theoretical and empirical basis (Sack et al., 2009), they essentially serve as 'baselines' for the FEF and hMT/V5+ location in individual subjects. Compared to these baselines, we evaluated the locations of TMS target points resulting from the Talairach-based and the CBA-based approach, quantified by their Euclidean distance from baseline, thus the individual fMRI hotspot.

\section{Individual Functional Localizers}

We first analyzed the data from the FEF and hMT/V5+ functional localizers on the individual level in order to determine the precise location of activation foci for all participants. For both tasks, localization was successful in all cases and individual Talairach coordinates are reported in Table 1. These coordinates were considered

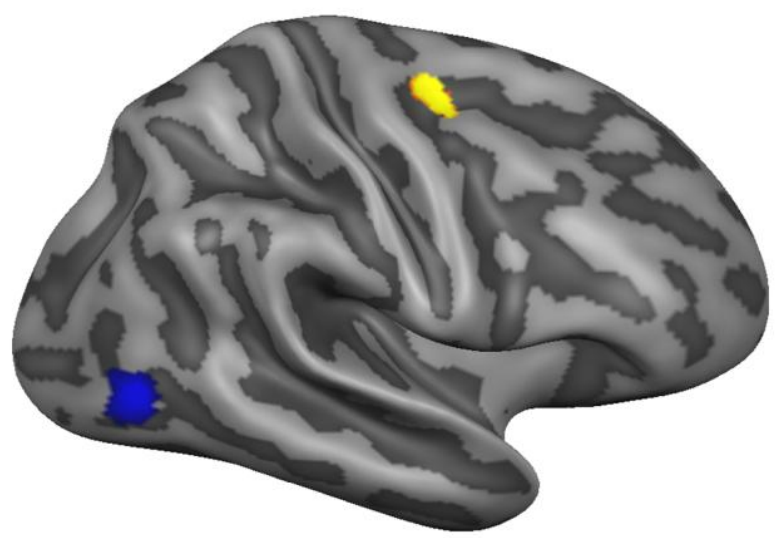

Figure 2. Group activation data projected on the inflated group average brain. The FEF activation hotspot (shown in yellow) was located at the junction of the pre-central sulcus and superior frontal sulcus. The contrast to identify FEF was "saccade > fixation". The hMT/V5+ activation hotspot (shown in blue) was located in the middle temporal complex. The contrast used to identify hMT/V5+ was "moving dots > stationary dots". For illustrative purposes, functional data in this figure have been masked so that occipital and parietal activation foci are absent. 
to represent the "true" locations of the respective areas for each participant that, in the ideal case, would be used as TMS target points. They thus served as reference points that were later used to calculate the relative displacement of TMS target points based on CBA and Talairach coordinates. It is noteworthy that there was a considerable variability across participants. To illustrate, we calculated the range for each dimension of the individual Talairach coordinates as a statistical measure of dispersion. The smallest interval (in millimeters) that included all the data in the $\mathrm{x}$, $\mathrm{y}$, and $\mathrm{z}$ direction was 16, 20, and 14 for FEF and 18, 27, and 13 for hMT/V5+, respectively. This already shows that a single set of Talairach coordinates is insufficient to accurately predict where a functional brain area is located at the individual level.

To allow comparisons with earlier studies, we also analyzed the data on the group level. As shown in Figure 2A, the FEF localizer showed consistent activation near the junction of the pre-central sulcus and superior frontal sulcus $(\mathrm{x}=25, \mathrm{y}=-$ $10, \mathrm{z}=53)$. The hMT/V5+ localizer was found to robustly activate the middle temporal complex $(\mathrm{x}=44, \mathrm{y}=-67, \mathrm{z}=0$; Figure $2 \mathrm{~B})$. This closely matches previously reported FEF (e.g. Amiez \& Petrides, 2009; Paus, 1996) and hMT/V5+ (e.g. Tootell et al., 1995) positions and confirms the adequacy of our functional localizers.

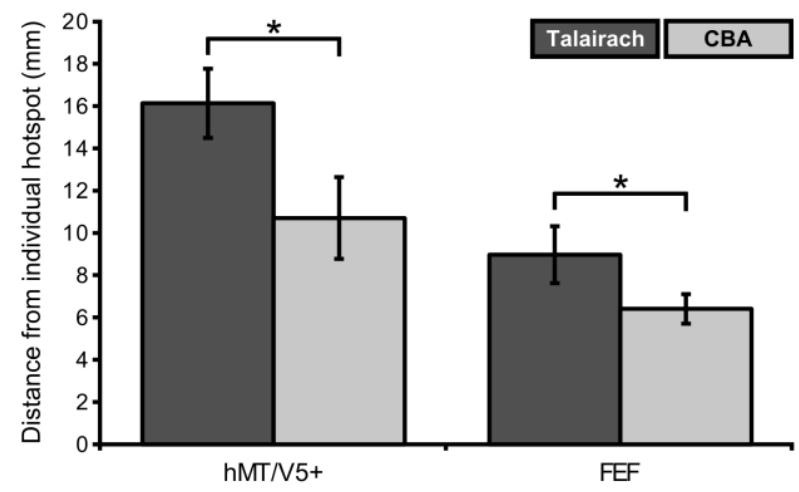

Figure 3. Average localization precision of the CBA-based and Talairach-based approach. Each approach is evaluated by calculating the Euclidean from the individual functional hotspot. For FEF and hMT/V5+, the CBA-based approach outperformed localization based on Talairach coordinates. Differences marked with an asterisk are statistically significant at an alpha level of 0.05 and error bars depict standard errors. 


\section{Cortex Based Alignment versus Talairach Coordinates}

We then assessed the precision of our proposed localization approach, which uses CBA to account for macro-anatomical variability across subjects, and determined the improvement of localization compared to traditional Talairach coordinates. With individual functional localizers serving as baselines, the critical question is how close each approach comes to these optimal individual hotspots. A repeatedmeasures ANOVA on the Euclidean distance from the individual hotspot was performed with localization approach (CBA and Talairach) and brain area (FEF and hMT/V5+) as within subject factors. This revealed a significant main effect of
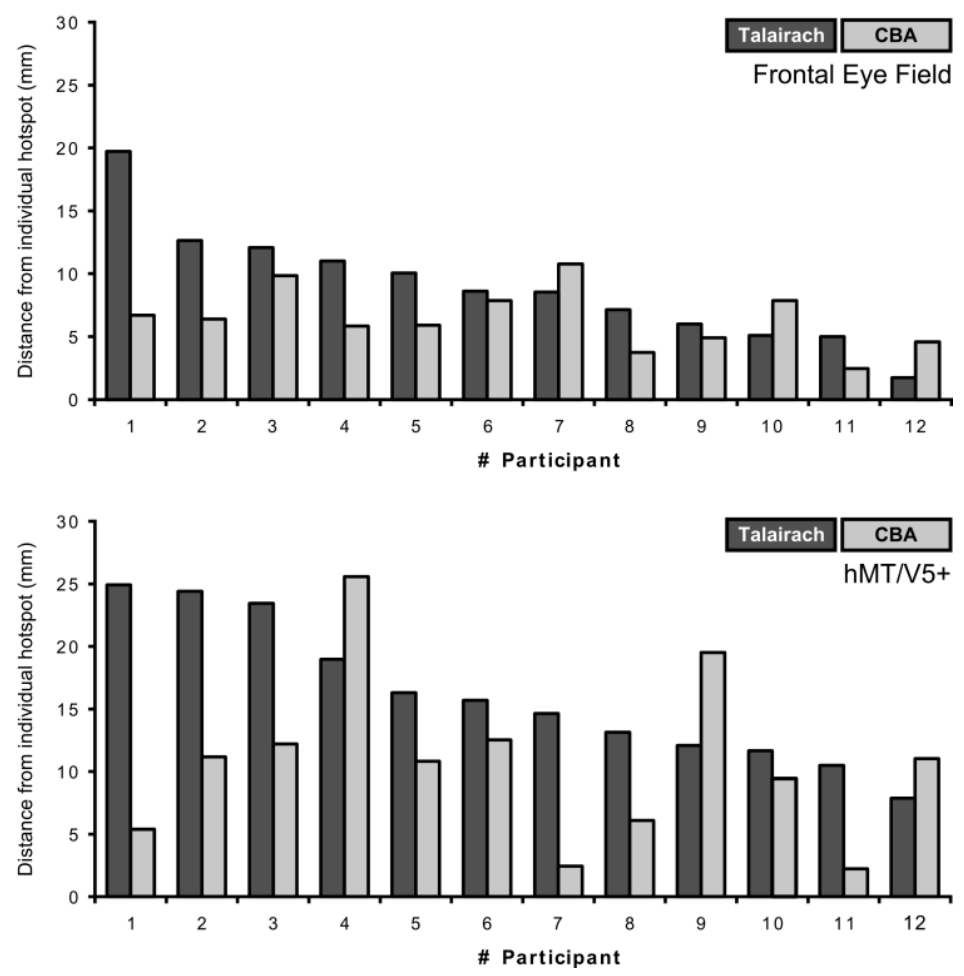

Figure 4. Localization precision of the CBA-based and Talairach-based approach on the individual level. For FEF (upper panel) and hMT/V5+ (lower panel), the CBA-based approach outperformed localization based on Talairach coordinates in 9 out of 12 participants. In each plot, participants are sorted, from large to small, according to the distance of the TMS target point based on Talairach coordinates from the individual functional hotspot. Consequently, there is no systematic correspondence of participant numbers between FEF and hMT/V5+ data. 


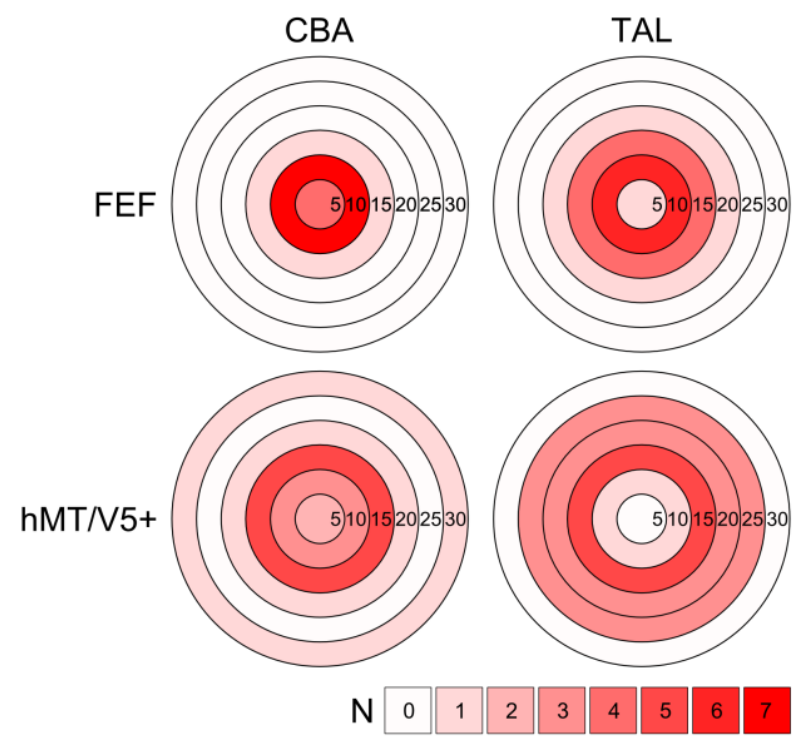

Figure 5. Distribution of the data for each brain area and localization approach. For FEF and hMT/V5+, the CBA-based approach brought more participants within the effective range of TMS compared to targeting based on Talairach coordinates. Data are binned at a $5 \mathrm{~mm}$ resolution and frequency counts are color-coded as indicated in the figure legend.

localization approach $\left(F_{(1,11)}=9.580, p=.010\right)$ and brain area $\left(F_{(1,11)}=10.964\right.$, $p=.007)$ but no interaction $\left(F_{(1,11)}=1.061, p=.325\right)$.

The main effect of localization approach resulted from higher precision of the CBA-based approach compared to the Talairach-based approach. On average, our approach improved localization by $4.0 \mathrm{~mm}(S E M=1.3)$. Specifically, the mean displacement (in millimeters) of TMS target points based on CBA and Talairach coordinates was 6.4 and 9.0 for FEF and 10.7 and 16.1 for hMT/V5+, respectively (Figure 3). Planned comparisons (paired-samples t-tests, one-tailed) confirmed that these improvements were significant for FEF $\left(t_{(11)}=1.975, p=.037\right)$ and hMT/V5+ $\left(t_{(11)}=2.294, p=.021\right)$. The main effect of brain area resulted from generally better localization of FEF compared to hMT/V5+ most likely due to differences in variability across participants as mentioned above and the degree of functionalanatomical correspondence (Frost \& Goebel, 2012). 


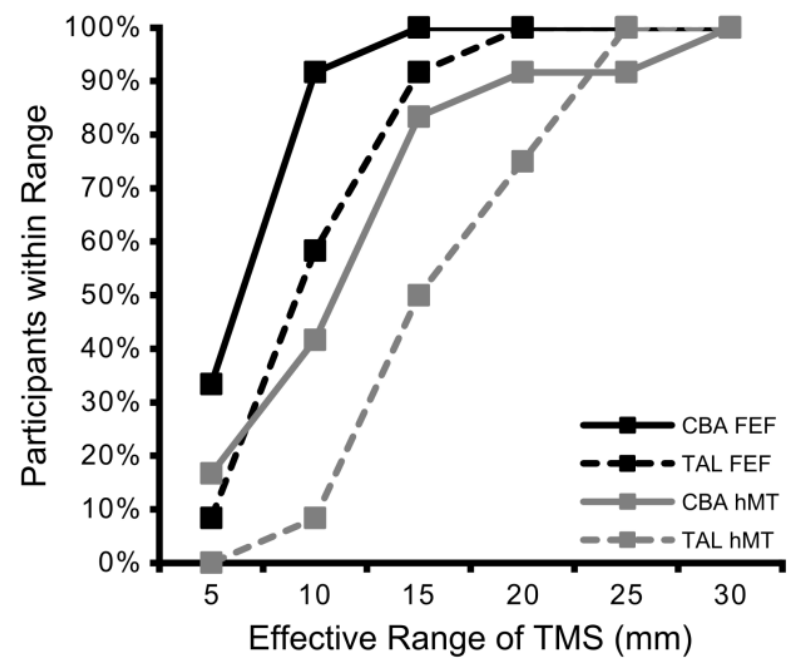

Figure 6. Proportion of participants within the effective range of TMS for each brain area and localization approach. For FEF and hMT/V5+, the CBA-based approach brought more participants within the effective range of TMS compared to targeting based on Talairach coordinates independent of the assumed effective range of TMS. Data have are binned at a $5 \mathrm{~mm}$ resolution and the performance of each approach can be evaluated at different cut-off values ranging from $5 \mathrm{~mm}$ to $30 \mathrm{~mm}$.

Because the effectiveness of TMS requires precise localization of TMS target points on the individual level, we further explored the data on a single subject basis. Ideally, the CBA-based approach reduces the distance from the individual hotspot not only on the group level but also in the majority of subjects compared to the Talairach-based approach. As shown in Figure 4, individual localization of FEF and hMT/V5+ was improved by our CBA-based approach in 9 out of 12 participants.

Finally, we evaluated the distribution of the data in the context of the spatial resolution of TMS. To avoid assuming a specific distance value where TMS is considered to be effective, we created bins with a width of $5 \mathrm{~mm}$ each (representing the distance from the individual hotspots) and counted the number of individual TMS target points that fell within these intervals. As can be seen in Figure 5, the CBA-based approach not only brought TMS target points closer to the center than the Talairach-based approach on the group level but also pulled a greater number of participants within the effective range of TMS. For example, assuming that the TMS coil should be within $15 \mathrm{~mm}$ from the true location of hMT/V5+ in 
order to have an effect, the Talairach-based approach would have been effective in only $50.0 \%$ of the participants whereas the CBA-based approach would have increased this number to $83.3 \%$. Importantly, this advantage of the CBA-based approach was not specific to any particular cut-off value but present irrespective of the assumed effective range of TMS (Figure 6).

\section{Discussion}

The success of TMS experiments critically depends on accurate TMS coil positioning. While functional localization based on individual fMRI data provides best results, we propose a novel method for TMS coil positioning that outperforms alternative approaches, such as targeting based on Talairach coordinates, but still only requires individual anatomical data as well as pre-existing functional group data. Our CBA-based approach should therefore be the method of choice either when individual functional data cannot be obtained or experimental factors argue against it (e.g. training or surprise effects).

Our results demonstrate that localization of TMS target points can be significantly improved compared to Talairach-based targeting when utilizing whole-brain alignment schemes that take the macro-anatomical differences between subjects into account. By incorporating already existing functional group data that are aligned in surface space, the proposed approach takes full advantage of the extent of functional-anatomical correspondence. This is in contrast to localization based on Talairach (or MNI) coordinates where this source of variability is completely ignored and alignment is done in volume space with only few anatomical landmarks serving as reference points. As a side note, we did not evaluate other (non-linear) volumetric alignment approaches such as SPM DARTEL (Ashburner, 2007) and FSL FNIRT (Andersson, Smith, \& Jenkinson, 2008). To the best of our knowledge, they have not been formally examined in the context of TMS coil positioning but similar benefits as our CBA-based approach could be expected. A direct comparison of all available non-linear whole-brain alignment schemes is beyond the scope of the 
present article. Here, we introduce our CBA-based approach and empirically demonstrate its benefits compared to what is common practice in the TMS community. The greatest advantage of the CBA-based approach is the balance between efficiency and accuracy. It is sufficient to obtain anatomical data for each participant, keeping measurement costs at a minimum (if anatomical data are not present already), while localization errors are significantly reduced compared to Talairach-based targeting. The direct comparison of the CBA-based and Talairachbased approach revealed that the CBA-based approach performed better in the majority of cases with improvements of a few millimeters on average. As will be discussed below, even these relatively small differences can be of practical relevance in context of a TMS experiment.

Previous work strongly suggests that the difference in accuracy between the CBA-based and Talairach-based approach reported here would translate into differences in effect size and statistical power. Sack et al. (2009) directly compared TMS-induced changes in task behavior using different approaches for TMS coil positioning, namely targeting based on 1) individual functional localizer, 2) individual brain anatomy, 3) Talairach coordinates, and 4) EEG electrode positions. Results showed that the number of participants required for a TMS effect to reach significance, as well as the risk of false negatives (type II error), can increase dramatically when localization accuracy is suboptimal. Based on this statistical argument, it is recommended to always use the best TMS coil positioning approach available. Moreover, previous work has shown that spatial displacements of the TMS coil in the order of millimeters can abolish behavioral effects altogether (Sack et al., 2006). In our view, the CBA-based approach should be the method of choice when individual functional localization is impossible.

The widespread use of Talairach space in neuroimaging has certainly contributed to the popularity of Talairach coordinates in TMS coil positioning. Transforming individual data to a common space is a crucial pre-requisite for most group analyses and, importantly, allows comparisons across experiments. Group activation data can be reduced to a simple set of coordinates, which also allows their easy use in a separate TMS experiment. However, the limitations of data representation in 
Talairach space have long been recognized and advanced whole-brain alignment schemes are more and more used in the neuroimaging community. In this sense, we propose that the TMS community should follow this development and take advantage of these improvements in the context of TMS coil positioning, as we demonstrate here.

We used a recently developed functional probabilistic atlas for the present study (Frost \& Goebel, 2012). This atlas contains functional probabilistic group maps of 10 brain areas and will soon be publicly available. By creating a probabilistic atlas one is able to identify the degree to which functional areas "respect" macroanatomical landmarks. Functional areas which are strongly bound to macro-anatomy are represented in the atlas as a small patch of cortical surface where there is a high likelihood that any new subject's functional area would be in the same anatomical location. By aligning a brain to this atlas brain, these probabilities can be used in subjects for whom functional data is not available. One must note however that these maps only represent the probabilistic location of the functional area. Although they are derived from functional data one cannot say, with complete certainty, that a particular functional area will occupy the same patch of cortex in all.

Finally, even though the CBA-based approach improves TMS coil positioning compared to Talairach-based targeting, our results also show that localization is still far from perfect. It is important to realize that this remaining localization error may still be decisive for finding a significant TMS effect in a given subject or patient. This remaining localization error of the CBA approach is brought about by a couple of general limitations that are inherent to any approach not using individual functional data and that can also not be overcome by using the here proposed CBAbased approach. First, the core of our approach is to improve localization of functional brain areas by incorporating macro-anatomical information. This obviously requires some degree of functional-anatomical correspondence. If the location of any given functional brain area is in no way related to the available anatomical data, then it is impossible to make any predictions based on anatomy alone. In such cases, nothing but individual functional localizers will be successful. Second, there is always the possibility of idiosyncrasies, that is, participants with 
functional brain areas that do not conform to regularities found in brain anatomy on the group level. This obviously cannot be captured with our approach, as these cases will be poorly localized, even with anatomical alignment. Again, nothing but individual functional localizers will be successful.

In conclusion, we propose a novel approach of TMS coil positioning that significantly improves localization accuracy compared to alternative approaches in situations where individual functional localization is not possible but pre-existing functional group data are available. We demonstrated the benefits of accounting for macro-anatomical variability across subjects for two brain areas, namely FEF and hMT/V5+. Consequently, we suggest that the TMS community could embrace the development of whole brain alignment schemes from the field of neuroimaging to improve TMS coil positioning.

\section{Acknowledgements}

This work was supported by the European Research Council under the European Union's Seventh Framework Programme (FP7/2007-2013) / ERC Grant agreement (263472 to A.T.S.), and the Netherlands Organization for Scientific Research (40007-230 to A.T.S \& E.F.). 


\section{References}

Amiez, C., \& Petrides, M. (2009). Anatomical organization of the eye fields in the human and non-human primate frontal cortex. Progress in Neurobiology, 89(2), $220-230$.

Andersson, J. L. R., Smith, S. M., \& Jenkinson, M. (2008). FNIRT - FMRIB's nonlinear image registration tool. Proceedings of the 14th Annual Meeting of the Organization for Human Brain Mapping.

Ashburner, J. (2007). A fast diffeomorphic image registration algorithm. NeuroImage, 38(1), 95-113.

Cohen Kadosh, R., Cohen Kadosh, K., Schuhmann, T., Kaas, A., Goebel, R., Henik, A., \& Sack, A. T. (2007). Virtual dyscalculia induced by parietal-lobe TMS impairs automatic magnitude processing. Current Biology, 17(8), 689-93.

Deng, Z.-D., Lisanby, S. H., \& Peterchev, A. V. (2012). Electric field depth-focality tradeoff in transcranial magnetic stimulation: Simulation comparison of 50 coil designs. Brain Stimulation, 6(1), 1-13. .

Fischl, B., Sereno, M. I., \& Dale, A. M. (1999). Cortical Surface-Based Analysis II: Infaltion, Flattening, and a Surface-Based Coordinate System. NeuroImage, 9(2), 195-207.

Fischl, B., Sereno, M. I., Tootell, R. B., \& Dale, A. M. (1999). High-resolution intersubject averaging and a coordinate system for the cortical surface. Human Brain Mapping, 8(4), 272-84.

Frost, M. A., \& Goebel, R. (2012). Measuring structural-functional correspondence: spatial variability of specialised brain regions after macro-anatomical alignment. NeuroImage, 59(2), 1369-1381.

Goebel, R., Esposito, F., \& Formisano, E. (2006). Analysis of functional image analysis contest (FIAC) data with brainvoyager QX: From single-subject to 
cortically aligned group general linear model analysis and self-organizing group independent component analysis. Human Brain Mapping, 27(5), 392-401.

Hallett, M. (2000). Transcranial magnetic stimulation and the human brain. Nature, 406(6792), 147-150.

Herwig, U., Kölbel, K., Wunderlich, A. P., Thielscher, A., Von Tiesenhausen, C., Spitzer, M., \& Schönfeldt-Lecuona, C. (2002). Spatial congruence of neuronavigated transcranial magnetic stimulation and functional neuroimaging. Clinical Neurophysiology, 113(4), 462-8.

Herwig, U., Satrapi, P., \& Schönfeldt-Lecuona, C. (2003). Using the international 10-20 EEG system for positioning of transcranial magnetic stimulation. Brain Topography, 16(2), 95-99.

Jasper, H. H. (1958). The ten-twenty electrode system of the International Federation. Electroencephalography and Clinical Neurophysiology, 10, 371-375.

Kammer, T., Puls, K., Erb, M., \& Grodd, W. (2005). Transcranial magnetic stimulation in the visual system. II. Characterization of induced phosphenes and scotomas. Experimental Brain Research, 160(1), 129-140.

Pascual-Leone, A., Walsh, V., \& Rothwell, J. (2000). Transcranial magnetic stimulation in cognitive neuroscience - virtual lesion, chronometry, and functional connectivity. Current Opinion in Neurobiology, 10(2), 232-237.

Paus, T. (1996). Location and function of the human frontal eye-field: A selective review. Neuropsychologia, 34(6), 475-483.

Paus, T. (1999). Imaging the brain before, during, and after transcranial magnetic stimulation. Neuropsychologia, 37, 219-224.

Sack, A. T. (2006). Transcranial magnetic stimulation, causal structure-function mapping and networks of functional relevance. Current Opinion in Neurobiology, $16(5), 593-599$. 
Sack, A. T., Cohen Kadosh, R., Schuhmann, T., Moerel, M., Walsh, V., \& Goebel, R. (2009). Optimizing functional accuracy of TMS in cognitive studies: a comparison of methods. Journal of Cognitive Neuroscience, 21(2), 207-221.

Sack, A. T., Kohler, A., Linden, D. E. J., Goebel, R., \& Muckli, L. (2006). The temporal characteristics of motion processing in hMT/V5+: combining fMRI and neuronavigated TMS. NeuroImage, 29(4), 1326-35.

Salminen-Vaparanta, N., Noreika, V., Revonsuo, A., Koivisto, M., \& Vanni, S. (2012). Is selective primary visual cortex stimulation achievable with TMS? Human Brain Mapping, 33(3), 652-665.

Schönfeldt-Lecuona, C., Thielscher, A., Freudenmann, R. W., Kron, M., Spitzer, M., \& Herwig, U. (2005). Accuracy of Stereotaxic Positioning of Transcranial Magnetic Stimulation. Brain Topography, 17(4), 253-259.

Sparing, R., Buelte, D., Meister, I. G., Paus, T., \& Fink, G. R. (2008). Transcranial magnetic stimulation and the challenge of coil placement: a comparison of conventional and stereotaxic neuronavigational strategies. Human Brain Mapping, 29(1), 82-96.

Talairach, J., \& Tournoux, P. (1988). Co-Planar Stereotaxic Atlas of the Human Brain. New York: Thieme.

Thielscher, A., Reichenbach, A., Uğurbil, K., \& Uludağ, K. (2010). The cortical site of visual suppression by transcranial magnetic stimulation. Cerebral Cortex, 20(2), 328-338.

Thompson, B., Aaen-Stockdale, C., Koski, L., \& Hess, R. F. (2009). A double dissociation between striate and extrastriate visual cortex for pattern motion perception revealed using rTMS. Human Brain Mapping, 30(10), 3115-3126.

Tootell, R. B., Reppas, J. B., Kwong, K. K., Malach, R., Born, R. T., Brady, T. J., Rosen, B. R., et al. (1995). Functional analysis of human MT and related visual 
cortical areas using magnetic resonance imaging. The Journal of Neuroscience, 15(4), 3215-3230.

Walsh, V., \& Cowey, A. (2000). Transcranial magnetic stimulation and cognitive neuroscience. Nature Reviews Neuroscience, 1(1), 73-79.

Wassermann, E. M., Mcshane, L. M., Hallett, M., \& Cohen, L. G. (1992). Noninvasive mapping of muscle representations in human motor cortex. Electroencephalography and Clinical Neurophysiology, 85, 1-8. 



\section{Chapter 5}

\section{Pre-Stimulus Sham TMS Facilitates Target Detection}

Based on:

Duecker, F., \& Sack, A. T. (2013). Pre-Stimulus Sham TMS Facilitates Target Detection. PLOS ONE, 8(3): e57765. 


\section{Abstract}

Transcranial magnetic stimulation (TMS) allows non-invasive manipulation of brain activity during active task performance. Because every TMS pulse is accompanied by non-neural effects such as a clicking sound and somato-sensation on the head, control conditions are required to ensure that changes in task behavior are indeed due to the induced neural effects. However, the non-neural effects of TMS in the context of a given task performance are largely unknown and, consequently, it is unclear what constitutes a valid control condition. We explored the non-neural effects of TMS on visual target detection. Participants received single pulse sham TMS to each hemisphere at different time points prior to target appearance during a visual target detection task. It was hypothesized that the clicking sound of a sham TMS pulse differentially affects performance depending on the location of the coil and the timing of the pulse.

Our results show that, first, sham TMS caused a facilitation of reaction times when preceding the target stimulus by 150,200 , and $250 \mathrm{~ms}$, whereas earlier and later time windows were not effective. Second, positioning the TMS coil ipsilateral instead of contralateral relative to the target stimulus improved reaction times. Third, infrequent noTMS trials that were interleaved with sham TMS trials had oddball-like properties resulting in increased reaction times during noTMS.

The clicking sound produced by sham TMS influences task performance in multiple ways. These non-neural effects of TMS need to be controlled for in TMS research and the present findings provide an empirical basis for deciding what constitutes a valid control condition. 


\section{Introduction}

Transcranial magnetic stimulation (TMS) is a widely used method in neuroscience that allows non-invasive manipulation of brain activity in healthy human volunteers by exposing the brain to a rapidly changing magnetic field. Next to the intended neural effects of TMS, every TMS pulse produces a distinct clicking sound and sensations on the head, and thus "non-neural" side effects, that potentially also systematically influence task behavior. Obviously, there is a strong need for appropriate control conditions in order to ensure that effects of interest are indeed the result of direct TMS-induced brain activity changes and not a consequence of the non-neural side effects of TMS. Before going into further detail, we would like to comment on the terminology introduced here. The dichotomy of 'neural' versus 'non-neural' effects could be regarded as being misleading or even incorrect. Of course, the clicking sound of the TMS coil is processed by the brain. In this sense, all behavioral effects caused by a TMS pulse are ultimately neural, no matter how they arise. However, when focusing on the immediate (physical) properties of a TMS pulse, 'neural' points to the changes in brain activity as they are caused by the magnetic field of the TMS pulse. In contrast, 'non-neural' is meant to refer to the sound and vibration of the TMS coil which are not per se related to the TMSinduced perturbations of brain activity.

There are different strategies of controlling for non-neural effects of TMS and the choice of control conditions often depends on the research question and experimental design (De Graaf \& Sack, 2011). In general, the idea is to demonstrate the specificity of results so that the same outcome is not observed under different TMS conditions. To give a few examples, TMS is commonly applied to multiple brain areas in order to show that the results are specific to one stimulation site. This can either be achieved by stimulation of brain areas that are differentially involved in a particular task or process, or by comparing the experimental condition to TMS over the vertex which should have no neural effect (spatial specificity). Similarly, when applying TMS at different time points, temporal information about the involvement of brain areas can be obtained aiming to reveal that the observed effects depend on the precise timing of stimulation (temporal specificity). Finally, there are 
sham TMS coils that mimic real TMS coils by producing the same clicking sound, and sometimes even sensations on the head, but do not cause any change in neural activity. Again, the idea is that a comparison between sham TMS and real TMS reveals the unique contribution of the neural effect of TMS on behavior. Importantly, the underlying assumption in all these cases is that the non-neural side effects of TMS are unspecific and therefore do not cause the behavioral differences between conditions. Although this assumption is vital for the interpretation of many TMS findings, only few studies have investigated the non-neural effects of TMS on behavior.

Regarding temporal specificity, there are few studies that report intersensory facilitation when a TMS pulse is administered in close temporal proximity to a target stimulus (Marzi et al., 1998; Sawaki, Okita, Fujiwara, \& Mizuno, 1999; Terao et al., 1997). This indicates that the clicking sound of a TMS pulse can indeed confound results when comparing different time points of stimulation. Similarly, subjective ratings of discomfort due to TMS have been found to positively correlate with error rate on a working memory task (Abler et al., 2005). However, to the best of our knowledge, other potential side effects have not been investigated yet. For the most part, it therefore appears an open empirical question whether the non-neural TMS side effects such as clicking sound of the TMS coil are indeed unspecific for task performance. This makes it difficult to decide on an empirical basis what constitutes a valid control condition. Next to the effects mentioned above, it might very well be that also spatial and other temporal non-neural aspects of a TMS pulse selectively modulate task performance and thus create behavioral specificity that could then be falsely interpreted as a direct neural effect of TMS, i.e. a false positive finding. Similarly, such a potential behavioral specificity of the non-neural effects of TMS might also work against the intended TMS-induced neural effects, leading to false null findings.

Here, we explored the non-neural effects of TMS on visual target detection. To this end, we applied sham TMS over the left and right hemisphere at different time points prior to target appearance. We aimed to address three basic empirical questions that have direct relevance for many TMS studies and provide guidance in designing appropriately controlled TMS experiments. First, we investigated whether 
a sham TMS pulse serves as a warning signal that transiently facilitates target detection. Many studies have shown that auditory stimuli can improve subsequent detection of visual targets in particular due to two different processes, namely temporal orienting and changes in phasic arousal (Hackley, 2009; Niemi \& Näätänen, 1981). We hypothesized that the clicking sound of an appropriately timed TMS pulse has a similar effect as reflected by decreased reaction times for visual target detection. Second, we explored the possibility that a lateralized TMS pulse automatically pulls spatial attention to the corresponding side of space. Almost all TMS studies apply TMS to brain areas away from the mid-sagittal line and, consequently, the clicking sound can be perceived as originating from one hemifield. Analogous to a cross-modal exogenous cueing task, we hypothesized that a TMS pulse facilitates target detection in the ipsilateral hemifield due to stimulus-driven shifts of spatial attention (Farah, Wong, Monheit, \& Morrowt, 1989; Schmitt, Postma, \& De, 2010; Spence \& Driver, 1997). Finally, it is common to include trials without TMS that are sometimes used as a control condition even though they might not reflect proper baseline performance. We hypothesized that performance on noTMS trials is context-dependent. Infrequent noTMS trials that are interleaved with sham TMS trials are unexpected and might have oddball-like properties resulting in increased reaction times compared to blocked noTMS trials (Audley, 1973; Kirby, 1980). Taken together, sham TMS is hypothesized to specifically influences task behavior in multiple ways despite the absence of neural effects and, consequently, can reveal factors that need to be controlled for when conducting real TMS experiments in order to avoid false positive or false negative findings.

\section{Methods and Materials}

\section{Participants}

Eighteen participants (12 female, aged 19 to 26) were recruited from the student population of Maastricht University. All had normal or corrected-to-normal vision and were right-handed. The research question and hypotheses remained unknown to 
the participants until the end of the experiment. The study was approved by the medical-ethical committee of the University Medical Center, Maastricht, the Netherlands. All participants gave written informed consent prior to participation and were screened for TMS experimentation safety by a medical supervisor.

\section{Stimuli and Task}

Participants performed a simple detection task requiring a single button press whenever a target stimulus was presented irrespective of target location (see Figure 1). A fixation cross was continuously presented at the centre of the screen and Gabor patches served as target stimuli that were shown for $100 \mathrm{~ms}$ either left or right of the fixation cross at 7 degrees eccentricity (spatial frequency $=1.5$ cycles per degree; envelope standard deviation $=0.75$ degrees; Michelson contrast $=60 \%$; random orientations). Participants were instructed to press the space bar on a standard keyboard with the right index finger as fast as possible as soon as the target stimulus appeared. Corrective feedback (an error message on the screen) was given in case of false alarms, misses, anticipatory responses (RT below $100 \mathrm{~ms}$ ), or very slow

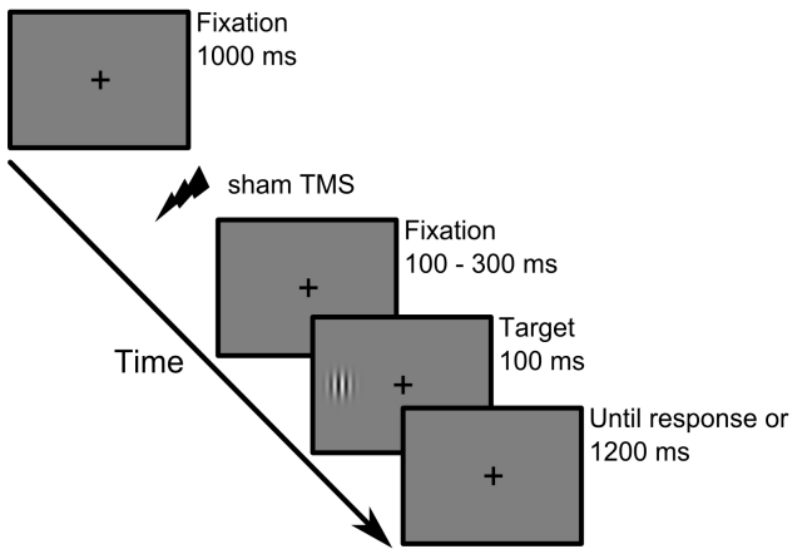

Figure 1. Example of a single trial of the detection task. Sham TMS was applied prior to target appearance and the time interval between TMS pulse and target onset was variable across trials ranging from 100 to $300 \mathrm{~ms}$. Target stimuli were briefly flashed either in the left or right hemifield and participants were instructed to respond as fast as possible with a single button press. 
responses (RT above $800 \mathrm{~ms}$ ). Stimuli were presented on a gamma-corrected 17" TFT screen (Samsung SyncMaster $931 \mathrm{DF}$ ) at $57 \mathrm{~cm}$ viewing distance with the head supported by a chin rest. The video mode was 1280 x 1024 at $60 \mathrm{~Hz}$ and background luminance was $25 \mathrm{~cd} / \mathrm{m}^{2}$. The Presentation software package (NeuroBehavioural Systems, Albany, CA) was used to control stimulus presentation and recording of behavioral responses.

\section{Procedure and Design}

The experiment consisted of one session per participant based on a full withinsubject design. During sham TMS trials, a single pulse was delivered to the right or left hemisphere at $300,250,200,150$, or $100 \mathrm{~ms}$ prior to target appearance. The exact position of the TMS coil on each participant's head was defined based on the International 10-20 system, namely C3 and C4. The TMS pulse contained no information about where the target stimulus would appear and catch trials were included to reduce anticipatory responses. We also included trials without TMS that were interleaved with sham TMS trials. For both stimulation sites, participants completed five blocks each consisting of 56 trials in randomized order resulting in 20 trials for each condition (including noTMS trials) and 40 catch trials. The TMS coil position was switched after five blocks and the order of conditions was counterbalanced across participants. Additionally, a single block of 80 trials without TMS was presented halfway through the experiment. After each block, participants could take a short break and received feedback about their average reaction time to ensure that they were motivated throughout the session.

\section{Transcranial Magnetic Stimulation}

Sham TMS pulses were applied using a Medtronic MagPro X100 stimulator (Medtronic Functional Diagnostics A/S, Skovlunde, Denmark) and a figure-of-eight placebo TMS coil (MC-P-B70; inner radius $=10 \mathrm{~mm}$; outer radius $=50 \mathrm{~mm}$ ). This coil is identical to a real TMS coil so that it produces the same clicking sound when 
discharging but a magnetic shield reduces the effective magnetic field by approximately $80 \%$. Stimulation intensity was set at $30 \%$ maximum stimulator output for all participants. At this intensity, a sham TMS pulse is too weak to produce any neural effect and hardly any sensation on the head can be perceived, except for weak vibrations of the coil. Participant received sham TMS over the left and right hemisphere, that is electrode position $\mathrm{C} 3$ and $\mathrm{C} 4$, respectively. Using a mechanical arm, the TMS coil was placed perpendicular on the head with the handle pointing posterior and slightly lateral.

\section{Eye Movement Control}

In order to exclude trials with breaks of central fixation, eye movements and eye blinks were measured using electrooculography (EOG). Data were recorded bipolarly from two pairs of $\mathrm{Ag} / \mathrm{AgCl}$ electrodes with a BrainAmp ExG system (BrainProducts GmbH, Munich, Germany). Electrodes were positioned at the outer canthus of each eye (horizontal EOG), above and below the right eye (vertical EOG), and on the mastoid behind the right ear (reference). The impedance of all electrodes was kept below $5 \mathrm{k} \Omega$. The EOG signal was digitized at $1000 \mathrm{~Hz}$, highpass filtered at $0.1 \mathrm{~Hz}$, and stored on disk using BrainVision Recorder (BrainProducts $\mathrm{GmbH}$, Munich, Germany). Offline data analysis was performed with BrainVision Analyzer (BrainProducts GmbH, Munich, Germany). Single trial data were visually inspected and all trials contaminated by eye movements or eye blinks were excluded from further analysis.

\section{Statistical Analysis}

After exclusion of all incorrect trials, outliers were removed from the data using the $1.5 \mathrm{x}$ IQR (interquartile range) criterion. Individual mean reaction times were then submitted to repeated measures ANOVAs. Depending on the hypothesis being tested, within-subject factors were sham TMS time window (5 levels: $-300,-250$, $-200,-150,-100)$, TMS coil position (2 levels: ipsilateral, contralateral), target 
location (2 levels: left, right), or context of no TMS trials (2 levels: interleaved, blocked). Effects of interest were then further explored with post-hoc contrasts and paired t-tests with correction for multiple comparisons by Fisher's LSD procedure.

\section{Results}

\section{Errors and Eye Movements}

All participants were able to perform the task at high levels of accuracy. Targets were correctly detected in $98.8 \%$ of all trials and false alarms only occurred in 4.4 $\%$ of the catch trials. A one-way repeated-measures ANOVA with sham TMS time window as within subject factor revealed a significant effect on accuracy $\left(F_{(4,14)}=\right.$ $6.395, p=.004)$. Post-hoc analysis showed a significant linear contrast $\left(F_{(1,17)}=\right.$ 24.232, $p$ <.001) resulting from decreasing accuracy with longer durations between sham TMS and target appearance due to slightly higher numbers of premature responses. Given that participants sometimes fail to withhold their response in anticipations of the target stimulus, this is not surprising because the likelihood of doing so increases with the length of the time interval. Nevertheless, accuracy was very high in all TMS time windows and never dropped below $96.9 \%$ on the group level. Similarly, accuracy in the interleaved and blocked trials without TMS was $99.4 \%$ and $99.0 \%$, respectively, and a paired samples t-test showed no significant difference $\left(t_{(17)}=.652, p=.523\right)$. In order to exclude any confounding effect of eye movements or eye blinks on reaction times, all trials with breaks of central fixation were discarded. In total, only $4.0 \%$ of all trials were contaminated ranging from $0.2 \%$ to $15.4 \%$ across participants. In sum, this indicates that participants had no difficulties performing the task and did not trade speed for accuracy so that the remaining analyses focused on reaction time differences. 


\section{No TMS Trials}

Target detection in the absence of TMS was performed in two different contexts, either interleaved with sham TMS or as a separate noTMS block. A repeatedmeasures ANOVA on mean reaction times with context (interleaved, blocked) and target location (left, right) as within-subject factors revealed a highly significant main effect of context $\left(F_{(1,17)}=248.257, p<.001\right)$, no effect of target location $\left(F_{(1,17)}=0.197, p=.663\right)$, and no interaction $\left(F_{(1,17)}=0.007, p=.933\right)$. The main effect of context resulted from slower reaction times for noTMS trials that were interleaved with sham TMS $(M=377 \mathrm{~ms}, S E=8.9)$ as compared with blocked noTMS trials $(M=295 \mathrm{~ms}, S E=8.0)$. This shows that noTMS trials do not necessarily reflect undistorted baseline performance but are modulated by context. In our case, when noTMS trials are interleaved with sham TMS they become infrequent events that have oddball-like properties (only one out of six targets was not preceded by a sham TMS pulse) resulting in slower reaction times. For that reason, only the blocked noTMS trials were used in subsequent analyses as a comparison for sham TMS related changes in target detection.

\section{Sham TMS Trials}

We then examined the effects of sham TMS on reaction times. A repeated-measures ANOVA with sham TMS time window (-300, -250, -200, -150, -100), TMS coil position (ipsilateral, contralateral), and target location (left, right) as within-subject factors revealed significant main effects of sham TMS time window $\left(F_{(4,14)}=9.548\right.$, $p<.001)$, TMS coil position $\left(F_{(1,17)}=25.615, p<.001\right)$, and target location $\left(F_{(1,17)}=5.528, p=.031\right)$. There were no significant interactions (all $p$ values $>.25$ ).

The main effect of sham TMS time window was best accounted for by a highly significant quadratic contrast $\left(F_{(1,17)}=30.177, p<.001\right)$, resulting from an $\mathrm{U}$-shaped reaction time curve, that is, intermediate time windows showed decreased reaction times relative to the earliest and latest time window. The same pattern of results was obtained when comparing all TMS time windows with blocked noTMS trials (Figure 2). Post-hoc pairwise comparisons revealed significantly decreased reaction 


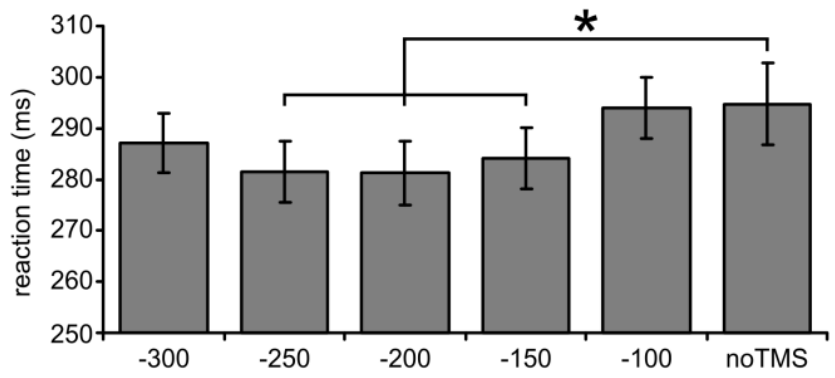

Figure 2. The effect of sham TMS time window on mean reaction time. A transient facilitation of reaction times was observed for intermediate time windows compared to blocked noTMS trials. Error bars show the standard error of the mean
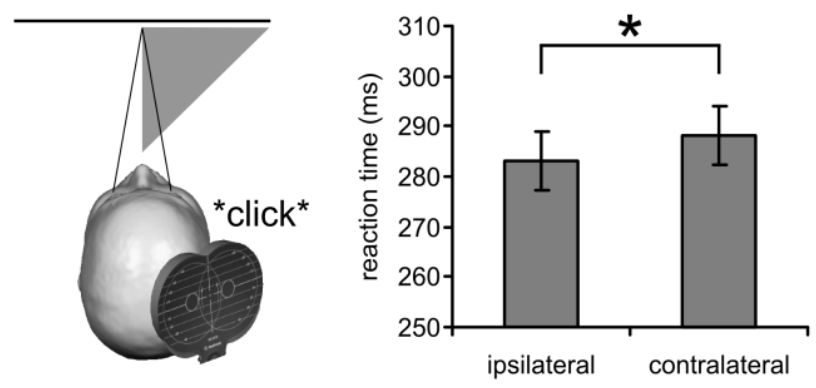

Figure 3. The effect of TMS coil position on mean reaction time. The clicking sound of a TMS pulse caused a shift of covert spatial attention to the corresponding side of space thereby facilitating target detection. Error bars show the standard error of the mean

times when the sham TMS pulse preceded the target by $250 \mathrm{~ms}\left(t_{(17)}=2.673\right.$, $p=.016), 200 \mathrm{~ms}\left(t_{(17)}=2.667, p=.016\right)$, and $150 \mathrm{~ms}\left(t_{(17)}=2.507, p=.023\right)$. There was no significant effect of the earliest $\left(t_{(17)}=1.611, p=.126\right)$ and latest time window $\left(t_{(17)}=0.131, p=.897\right)$, that is, $300 \mathrm{~ms}$ and $100 \mathrm{~ms}$ prior to target appearance, respectively. This suggests that a sham TMS pulse serves as a warning stimulus that transiently facilitates target detection.

The main effect of TMS coil position resulted from faster reaction times when the sham TMS coil was ipsilateral $(M=283 \mathrm{~ms}, S E=5.8)$ instead of contralateral $(M=288 \mathrm{~ms}, S E=5.9)$ to the target stimulus (Figure 3$)$. This indicates that a 
lateralized sham TMS pulse causes a reflexive shift of covert spatial attention to the corresponding side of space and thereby facilitating target detection.

Finally, the main effect of target location was caused by faster reaction times for target stimuli in the right hemifield $(M=284 \mathrm{~ms}, S E=6.1)$ compared with the left hemifield $(M=288 \mathrm{~ms}, S E=5.8)$ probably because participant used their right hand to respond (Simon effect, Simon \& Rudell, 1967).

\section{Discussion}

The non-neural effects of TMS on behavior are largely unknown and, consequently, it is unclear what constitutes a valid control condition. It is often assumed that the used control conditions show no behavioral specificity in the context of a given experimental task. Surprisingly, this assumed lack of specificity of, e.g., sham TMS has rarely been tested empirically. Here, we investigated the effects of pre-stimulus sham TMS on target detection and provide evidence that sham TMS produces systematic and specific changes in target detection reaction times. Depending on the experimental design and research question, these specific effects of sham TMS can lead to false positive or false negative results. Our findings thus have direct implications for many TMS experiments, and could be used to guide the choice of an appropriate control condition in the context of a concrete research question. Specifically, we tested three hypotheses of sham TMS-induced behavioral specificity that were all confirmed by our present findings.

Our first hypothesis was that an appropriately timed sham TMS pulse serves as an acoustic warning stimulus that facilitates visual target detection due to temporal orienting and/or increased levels of phasic arousal. Indeed, our results show decreased reaction times when a sham TMS pulse precedes the target stimulus by 150, 200, or $250 \mathrm{~ms}$ compared to our baseline condition whereas earlier and later time windows were not effective. The absence of an effect at 100 and $300 \mathrm{~ms}$ prior to target appearance suggests that the warning signal effect was mainly due to temporal orienting because of the following reasons. Once the sham TMS pulse is administered, the probability of target occurrence increases over time and, 
consequently, reaction times gradually become faster with increasing stimulus onset asynchronies (SOA). At $100 \mathrm{~ms}$, the target stimulus might still come as a surprise and the time to prepare for target appearance might be insufficient, explaining the absence of an effect. Following this line of thought, reaction times at the $300 \mathrm{~ms}$ time window should be fastest but the inclusion of catch trials in the current experiment increased uncertainty for the longest SOAs because the target might not be presented at all resulting in slower reaction times. Taken together, the present pattern of results seems to be best accounted for in terms of temporal orienting. However, a transient effect on phasic arousal cannot be excluded and might contribute to the observed facilitatory effects. This strongly suggests that the clicking sound of a TMS pulse acts as a warning signal and has similar properties to other kinds of warning stimuli (Hackley, 2009; Niemi \& Näätänen, 1981). This finding complements earlier reports of intersensory facilitation that occurs when the clicking sound of the TMS pulse temporally coincides with target appearance (Marzi et al., 1998; Sawaki et al., 1999; Terao et al., 1997). The time windows used in the present experiment were all prior to target appearance so that a direct comparison with these experiments is difficult. However, the absence of any facilitation at $100 \mathrm{~ms}$ before target onset suggests that the effects observed in our study do not reflect the same underlying mechanism. As argued above, an interpretation in terms of temporal orienting seems most likely. As a consequence, TMS experiments that compare different time points of stimulation cannot assume that the non-specific effects of TMS on behavior are constant across temporal conditions. Timedependent TMS effects therefore require a control condition that consists of either real or sham TMS at the same time points as the experimental condition. Only then the non-neural effects of TMS on temporal orienting and/or arousal are actually controlled for.

Our second hypothesis was that a sham TMS pulse can cause an automatic shift of covert spatial attention when applied away from the mid-sagittal line, thereby facilitating unilateral target detection. Indeed, improved reaction times were observed when the TMS coil was positioned ipsilateral instead of contralateral relative to the target stimulus. This is in agreement with findings from cross-modal cueing tasks where non-predictive lateralized auditory stimuli have been shown to 
speed detection of visual targets at the cued location (Farah et al., 1989; Schmitt et al., 2010; Spence \& Driver, 1997). In many cases, a distance effect is observed with cueing effects being stronger when the precise location of the auditory cue and visual target match. Our results show that cross-modal cueing effects even occur in case of spatial correspondence regarding hemifields. As a consequence, TMS experiments that compare different stimulation sites cannot assume that the nonneural effects of TMS on behavior are constant across conditions. The cueing effect reported here can only be controlled for when the TMS coil is positioned at a location that produces the same effects on spatial attention. The conditions under which this is the case cannot be inferred from the present study because we only investigated the difference between the left and right hemisphere. Nevertheless, it seems necessary to choose a stimulation site as control condition that lies in the same hemisphere and maybe even has as a similar degree of laterality. This also implies that vertex stimulation is not a suitable control condition for lateral real stimulation sites especially when tasks require a rigid control of the locus of spatial attention.

Our third hypothesis was that performance on trials without TMS depends on the context in which they are presented. More specifically, infrequent noTMS trials that are interleaved with sham TMS trials were expected to have oddball-like properties (Audley, 1973; Kirby, 1980). This was thought to result in increased reaction times compared to sham TMS trials and noTMS trials that are presented in separate blocks. Our results clearly show that reaction times on interleaved noTMS trials are strongly confounded and do not reflect proper baseline performance. Blocked noTMS trials, on the other hand, were indistinguishable from the earliest and latest sham TMS time window and, for our purposes, could be used for further analysis. In general, this strongly suggests that interleaved noTMS trials should not be used as baseline condition. They obviously do not control for the non-neural effects of TMS and, on top of that, are modulated by the presence of TMS trials. Since this modulation most likely depends on many aspects of the experimental design, the validity of noTMS trials as baseline condition is difficult to establish.

Taken together, our results provide insights into the specificity of non-neural effects of TMS on behavior. Importantly, this does not challenge the validity of 
sham TMS as a control condition. Quite the contrary, all the effects reported here most likely occur during real TMS as well. In this sense, our results demonstrate that sham TMS can be considered an appropriate control condition for at least some of the non-neural effects of TMS. However, the sham TMS coil used in this experiment does not produce sensations on the head that match those of real TMS. There might therefore be additional non-neural effects of TMS that remained undetected and require further investigation.

It has to be emphasized that the present study applied sham TMS always prior to target appearance. This makes our experiment particularly sensitive to the effects described above and it therefore remains unclear in how far similar non-neural effects of TMS can be observed with other stimulation parameters, tasks, and outcome measures. Of course, there is an abundance of TMS experiments that used sham TMS as a control condition and these could, in principle, be re-evaluated to discover additional non-neural side effects of TMS. While a full review of this matter is beyond the scope of this article, we examined previous publications from our own group in the light of the present findings. To begin with, reaction times on an angle judgment task were found to linearly increase across post-stimulus time windows when applying sham TMS suggesting that participant sometimes delay their response until a TMS pulse is administered (De Graaf, Jacobs, Roebroeck, \& Sack, 2009). Additionally, no TMS trials have repeatedly been associated with unusual slowing of reaction times (De Graaf, Cornelsen, Jacobs, \& Sack, 2011; de Graaf, Herring, \& Sack, 2011) resembling the context effect reported in the present study. However, there are also many studies that did not reveal changes in task behavior due to sham TMS. For example, pre-stimulus sham TMS over early visual cortex neither affected behavioral priming (Jacobs, De Graaf, Goebel, \& Sack, 2012) nor did we observe any significant effects on accuracy and awareness ratings in a visual masking paradigm (Jacobs, Goebel, \& Sack, 2012) whereas real TMS clearly revealed time-dependent modulations of task behavior. Similarly, Schuhmann et al. found no effect on picture naming latency when applying sham TMS over Broca's area after stimulus presentation (Schuhmann, Schiller, Goebel, \& Sack, 2012). Overall, it seems that the non-neural side effects of TMS depend on many factors and the present study should be seen as a starting point for identifying those 
conditions that are especially vulnerable to such effects.

In conclusion, we show that the clicking sound produced by a sham TMS coil acts as a warning signal, causes automatic shifts of spatial attention, and creates a context that influences noTMS trials. These factors are relevant for a broad range of experiments and the present findings therefore provide an empirical basis for deciding what constitutes an appropriate control condition to avoid or minimize both, chances for false positive as well as false negative TMS findings.

\section{Acknowledgements}

This work was supported by the European Research Council under the European Union's Seventh Framework Programme (FP7/2007-2013) / ERC Grant agreement (263472 to A.T.S.), and the Netherlands Organization for Scientific Research (40007-230 to A.T.S \& E.F.). We thank Elles Douven and Amber van der Meij for their assistance during data acquisition, our medical supervisor Cees van Leeuwen, and our independent physician Martin van Boxtel. 


\section{References}

Abler, B., Walter, H., Wunderlich, A., Grothe, J., Schönfeldt-Lecuona, C., Spitzer, M., \& Herwig, U. (2005). Side Effects of Transcranial Magnetic Stimulation Biased Task Performance in a Cognitive Neuroscience Study. Brain Topography, 17(4), 193-196.

Audley, R. J. (1973). Some Observations on Theories of Choice Reaction Time: Tutorial Review. In S. Kornblum (Ed.), Attention and Performance IV (pp. 509545). New York: Academic Press.

Farah, M. J., Wong, A. B., Monheit, M. A., \& Morrowt, L. A. (1989). Parietal Lobe Mechanisms of Spatial Attention: Modality-Specific or Supramodal? Neuropsychologiap, 27, 461-470.

De Graaf, T. A., Cornelsen, S., Jacobs, C., \& Sack, A. T. (2011). TMS effects on subjective and objective measures of vision: stimulation intensity and pre- versus post-stimulus masking. Consciousness and Cognition, 20(4), 1244-1255.

De Graaf, T. A., Herring, J., \& Sack, A. T. (2011). A chronometric exploration of high-resolution "sensitive TMS masking" effects on subjective and objective measures of vision. Experimental Brain Research, 209(1), 19-27.

De Graaf, T. A., Jacobs, C., Roebroeck, A., \& Sack, A. T. (2009). FMRI effective connectivity and TMS chronometry: complementary accounts of causality in the visuospatial judgment network. PloS one, 4(12), e8307.

De Graaf, T. A., \& Sack, A. T. (2011). Null results in TMS: from absence of evidence to evidence of absence. Neuroscience and Biobehavioral Reviews, 35(3), 871-877.

Hackley, S. A. (2009). The speeding of voluntary reaction by a warning signal. Psychophysiology, 46, 225-233. 
Jacobs, C., Goebel, R., \& Sack, A. T. (2012). Visual awareness suppression by prestimulus brain stimulation; a neural effect. NeuroImage, 59(1), 616-624.

Jacobs, C., De Graaf, T. A., Goebel, R., \& Sack, A. T. (2012). The temporal dynamics of early visual cortex involvement in behavioral priming. PloS ONE, 7(11), e48808.

Kirby, N. H. (1980). Sequential Effects in Choice Reaction Time. In A. T. Welford (Ed.), Reaction Times (pp. 129-172). London: Academic Press.

Marzi, C. A., Miniussi, C., Maravita, A., Bertolasi, L., Zanette, G., Rothwell, J. C., \& Sanes, J. N. (1998). Transcranial magnetic stimulation selectively impairs interhemispheric transfer of visuo-motor information in humans. Experimental brain research, 118(3), 435-438.

Niemi, P., \& Näätänen, R. (1981). Foreperiod and Simple Reaction Time. Psychological Bulletin, 89(1), 133-162.

Sawaki, L., Okita, T., Fujiwara, M., \& Mizuno, K. (1999). Specific and non-specific effects of transcranial magnetic stimulation on simple and go/no-go reaction time. Experimental Brain Research, 127(4), 402-408.

Schmitt, M., Postma, A., \& De, E. (2010). Interactions Between Exogenous Auditory and Visual Spatial Attention. The Quarterly Journal of Experimental Psychology, 53A(1), 105-130.

Schuhmann, T., Schiller, N. O., Goebel, R., \& Sack, A. T. (2012). Speaking of which: dissecting the neurocognitive network of language production in picture naming. Cerebral cortex, 22(3), 701-709.

Simon, J. R., \& Rudell, A. P. (1967). Auditory S-R compatibility: The effect of an irrelevant cue on information processing. Journal of Applied Psychology, 51(3), 300-304.

Spence, C., \& Driver, J. (1997). Audiovisual Links in Exogenous Covert Spatial Orienting. Perception \& Psychophysics, 59(1), 1-22. 
Terao, Y., Ugawa, Y., Suzuki, M., Sakai, K., Hanajima, R., Gemba-Shimizu, K., \& Kanazawa, I. (1997). Shortening of simple reaction time by peripheral electrical and submotor-threshold magnetic cortical stimulation. Experimental Brain Research, $115(3), 541-545$. 



\section{Chapter 6}

Time- and Task-Dependent Non-Neural Effects of Real and Sham TMS

Based on:

Duecker, F., de Graaf, T. A., Jacobs, C., Sack, A. T. (2013). Time- and TaskDependent Non-Neural Effects of Real and Sham TMS. PLoS ONE, 8(9), e73813. 


\section{Abstract}

Transcranial magnetic stimulation (TMS) is widely used in experimental brain research to manipulate brain activity in humans. Next to the intended neural effects, every TMS pulse produces a distinct clicking sound and sensation on the head which can also influence task performance. This necessitates careful consideration of control conditions in order to ensure that behavioral effects of interest can be attributed to the neural consequences of TMS and not to non-neural effects of a TMS pulse. Surprisingly, even though these non-neural effects of TMS are largely unknown, they are often assumed to be unspecific, i.e. not dependent on TMS parameters. This assumption is inherent to many control strategies in TMS research but has recently been challenged on empirical grounds.

Here, we further develop the empirical basis of control strategies in TMS research. We investigated the time-dependence and task-dependence of the nonneural effects of TMS and compared real and sham TMS over vertex. Critically, we show that non-neural TMS effects depend on a complex interplay of these factors. Although TMS had no direct neural effects, both pre- and post-stimulus TMS time windows modulated task performance on both a sensory detection task and a cognitive angle judgment task. For the most part, these effects were quantitatively similar across tasks but effect sizes were clearly different. Moreover, the effects of real and sham TMS were almost identical with interesting exceptions that shed light on the relative contribution of auditory and somato-sensory aspects of a TMS pulse.

Knowledge of such effects is of critical importance for the interpretation of TMS experiments and helps deciding what constitutes an appropriate control condition. Our results broaden the empirical basis of control strategies in TMS research and point at potential pitfalls that should be avoided. 


\section{Introduction}

Transcranial magnetic stimulation (TMS) is a non-invasive interference technique that is widely used in experimental brain research (Hallett, 2000; Pascual-Leone, Walsh, \& Rothwell, 2000; Sack, 2006; Walsh \& Cowey, 2000). By exposing the brain to a rapidly changing magnetic field, TMS allows manipulation of brain activity in human volunteers during task execution with excellent temporal resolution and high spatial precision. Next to the intended neural effects of TMS, every TMS pulse produces a distinct clicking sound and sensations on the head. The presence of these non-neural effects creates a strong need for appropriate control conditions. TMS experiments have to be designed in such a way that behavioral effects of interest can be attributed to the neural consequences of TMS and not to non-neural effects of a TMS pulse.

Even though appropriate control conditions are key to the interpretation of any TMS effect, there is a surprising lack of empirical evidence on the non-neural effects of TMS. Only in the late 1990s, this issue briefly captured attention with some studies reporting intersensory facilitation when a TMS pulse is administered in close temporal proximity to a target stimulus (Marzi et al., 1998; Nikouline, Ruohonen, \& Ilmoniemi, 1999; Sawaki, Okita, Fujiwara, \& Mizuno, 1999; Terao et al., 1997). Since then, the TMS community has rarely addressed the non-neural effects of TMS explicitly and rather developed control strategies based on theoretical grounds, mainly around the concept of specificity. The general idea is that a TMS effect is thought to be 'neural' when it is specific to a stimulation site, time point, or other parameters. The implicit assumption is that the non-neural effects of a TMS pulse lack such specificity and therefore do not produce differences between conditions.

Recently, we have challenged this assumption on empirical grounds (Duecker \& Sack, 2013). We investigated the non-neural effects of pre-stimulus TMS on target detection and revealed that the clicking sound of a sham TMS coil systematically influences task performance. First, we showed that a TMS pulse acts as a warning signal that facilitates reaction times to subsequent visual stimuli. Second, we found that a lateralized TMS pulse causes an automatic shift of spatial attention to the corresponding side of space. Importantly, the discovery of these non-neural effects 
of TMS is not per se problematic; they rather help deciding what constitutes a valid control condition. In the context of target detection, these findings demonstrated that comparing different stimulation sites or time points can be insufficient because these strategies might fail to dissociate neural from non-neural effects. Being aware of such effects, however, it is obviously possible to design experiments that control for these factors.

In the present study, we set out to further develop the empirical basis of control conditions in TMS research. To this end, we applied not only sham TMS, but now also real TMS over the vertex at not only pre-, but now also post-stimulus time windows. Moreover, aside from the sensory target detection task, we now also added a more cognitive 'angle judgment' task. We address three questions that have direct relevance for common control strategies in TMS research.

First, are there time-specific non-neural effects of single TMS pulses? By measuring a broad range of TMS time windows we aimed to replicate previously reported facilitatory non-neural effects of pre-stimulus TMS (Duecker \& Sack, 2013) and identify potentially specific non-neural effects of post-stimulus TMS. We hypothesized that post-stimulus TMS impairs task performance: the mere presence of the clicking sound of the TMS coil during each trial might create an expectancy that is violated when the TMS pulse occurs relatively late. This could result in delayed responses because participants 'wait' for the TMS pulse (De Graaf, Jacobs, Roebroeck, \& Sack, 2009).

Second, are the non-neural effects of TMS dependent on experimental task? Despite our previous findings in the context of target detection reported above, one could argue that others tasks that involve higher cognitive functions and require a more complex stimulus response mapping are immune to such effects. For that reason, we assessed the non-neural effects of TMS on two tasks, namely a target detection task and an angle judgment task. This comparison provides information about the generalizability of the non-neural effects of TMS and, importantly, also has direct implications for TMS experiments that employ control tasks in order to demonstrate specificity.

Third, are the non-neural effects of sham TMS comparable to the non-neural 
effects of real TMS? This question is particularly relevant for experiments predominantly relying on sham TMS to control for non-neural effects. We compared the effects of real and sham TMS over the vertex. Assuming that real TMS over the vertex does not produce any neural effects, this comparison allows assessing the effect of the clicking sound of the TMS coil on task performance and to dissociate this from the added influence of the somato-sensory effect of TMS.

\section{Methods and Materials}

\section{Participants}

Fourteen participants ( 8 female, aged 19 to 28) were recruited from the student population of Maastricht University. All had normal or corrected-to-normal vision and were right handed. The research question and hypotheses remained unknown to the participants until the end of the experiment. The study was approved by the medical-ethical committee of the University Medical Center, Maastricht, the Netherlands. All participants gave written informed consent prior to participation and were screened for TMS experimentation safety by a medical supervisor.

\section{Stimuli and Task}

We investigated the neural and non-neural effects of TMS over vertex on two different tasks, namely a detection task and an angle judgment task (see below). Stimuli were presented on a gamma-corrected 17" TFT screen (Samsung SyncMaster $931 \mathrm{DF}$ ) at $57 \mathrm{~cm}$ viewing distance with the head supported by a chin rest. The video mode was 1280 x 1024 at $60 \mathrm{~Hz}$ and background luminance was $25 \mathrm{~cd} / \mathrm{m}^{2}$. The Presentation software package (NeuroBehavioural Systems, Albany, CA) was used to control stimulus presentation and recording of behavioral responses. For both tasks, responses were given on the numeric keypad of a standard keyboard. 


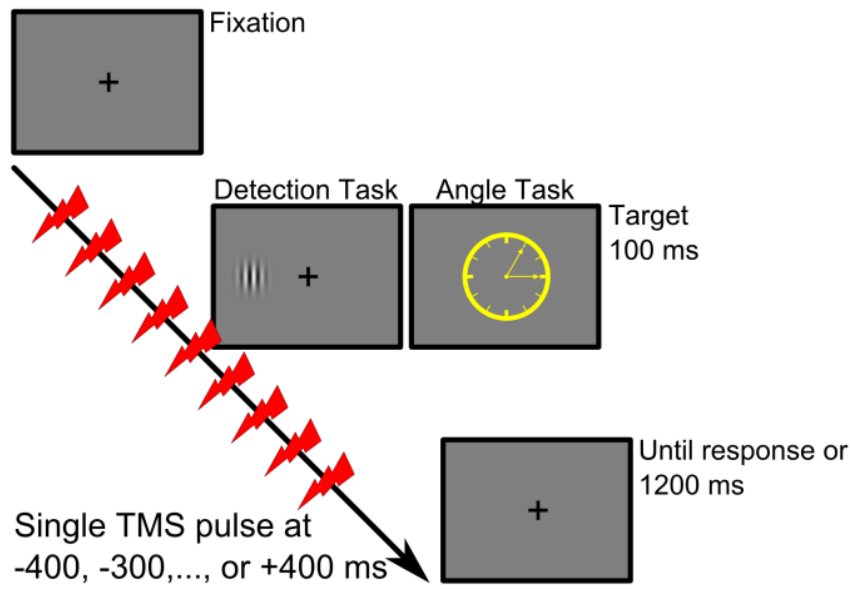

Figure 1. Example of a single trial for each task. Real or sham TMS was applied at one out of nine possible time points during each trial covering pre- and post-stimulus time windows ranging from -400 $\mathrm{ms}$ to $+400 \mathrm{~ms}$ relative to stimulus onset.

The detection task (Figure 1) required participants to perform a single button press whenever a target stimulus was presented irrespective of target location. A fixation cross was continuously presented at the centre of the screen and Gabor patches served as target stimuli that were shown at $400 \mathrm{~ms}$ after trial onset for $100 \mathrm{~ms}$ either left or right of the fixation cross at 7 degrees eccentricity (spatial frequency $=1.5$ cycles per degree; envelope standard deviation $=0.75$ degrees; Michelson contrast $=60 \%$; random orientations). Participants were instructed to press the "NUM1" key with the right index finger as fast as possible as soon as the target stimulus appeared. The average inter-trial interval was four seconds with a jitter of $\pm 500 \mathrm{~ms}$.

The angle judgment task (Figure 1) required participants to judge the angle formed by two arrows similar to hour and minute hands on an analogue clock face (De Graaf et al., 2009; de Graaf, Roebroeck, Goebel, \& Sack, 2010; Sack et al., 2002, 2007). On each trial, one out of twenty-four pre-generated clock faces with a diameter of 10 degrees of visual angle was presented at $400 \mathrm{~ms}$ after trial onset for $100 \mathrm{~ms}$ at the centre of the screen. The angle formed by the clock hands was 30, 60, 90, or 120 degrees and participants had to discriminate small (30 and 60 degrees) 
from large angles (90 and 120 degrees). Participants were instructed to press the "NUM1" key with their right index finger for small angles and the "NUM2" key with their right middle finger for large angles (90 and 120 degrees). A fixation cross was continuously presented between stimuli at the center of the screen. As above, the average inter-trial interval was four seconds with a jitter of $\pm 500 \mathrm{~ms}$.

\section{Procedure and Design}

A within-subject design was employed in which all participants completed two TMS sessions on non-consecutive days. At the beginning of each session, participants received detailed instructions for the detection task and angle judgment task and completed 20 practice trials to get acquainted with the stimuli, timing, and required responses. During the main part of the experiment, participants performed blocks of both tasks in alternation, receiving real TMS in the first session and sham TMS in the second session, or vice versa (counterbalanced across participants). During all TMS trials, a single TMS pulse was delivered to the vertex at one out of nine possible TMS time windows (ranging from -400 to $+400 \mathrm{~ms}$ in steps of $100 \mathrm{~ms}$ ) time-locked to stimulus onset. The timing of the TMS pulse was task-irrelevant and provided no information about the properties of the stimulus. We also included trials without TMS that were interleaved with TMS trials. For both tasks, participants completed four blocks each consisting of 60 trials in randomized order resulting in 24 trials for each condition (including noTMS trials). Additionally, we included blocks of 24 trials without TMS at the beginning, halfway, and at the end of the experiment. Between blocks, participants could take a short break, were informed about which task was to come next, and whether or not TMS would be applied.

\section{Transcranial Magnetic Stimulation}

Single biphasic TMS pulses were applied using a Medtronic MagPro X100 stimulator (Medtronic Functional Diagnostics A/S, Skovlunde, Denmark) and a figure-of-eight TMS coil (MC-B70; inner radius $=10 \mathrm{~mm}$; outer radius $=50 \mathrm{~mm}$ ). 
The stimulation intensity when applying real TMS was set at $50 \%$ maximum stimulator output for all participants. For the sham stimulation, a placebo TMS coil was used (MC-P-B70) with the same mechanical outline and clicking sound when discharging but equipped with a magnetic shield that reduces the effective magnetic field by approximately $80 \%$. Here, the stimulation intensity was set at $30 \%$ maximum stimulator output in order to match the sound level produced by the real TMS coil. At this stimulation intensity, a sham TMS pulse is too weak to produce any neural effect and hardly any sensation on the head can be perceived, except for weak vibrations of the coil. Participant received real TMS and sham TMS over the vertex, that is electrode position $\mathrm{Cz}$ as defined by the International 10-20 system (Jasper, 1958). Using a mechanical arm, the TMS coil was placed on the head with the handle pointing posterior and the initial current direction going away from the handle.

\section{Statistical Analysis}

The analysis of reaction times was performed after exclusion of all incorrect trials and outliers were removed from the data using the $1.5 \times \mathrm{IQR}$ (interquartile range) criterion. We then submitted individual mean reaction times and accuracies to repeated measures ANOVAs. Depending on the hypothesis being tested, withinsubject factors were TMS time window (9 levels: -400, -300, -200, -100, 0, 100, 200, 300, $400 \mathrm{~ms}$ ), TMS type (2 levels: real, sham), task (2 levels: detection, angle judgment), or context of no TMS trials (2 levels: interleaved, blocked). In cases where the sphericity assumption was violated, we performed a Greenhouse-Geisser correction but for simplicity the degrees of freedom are reported uncorrected. Effects of interest were explored with paired t-tests with Bonferroni correction for multiple comparisons unless stated otherwise. 


\section{Results}

\section{Difference between Blocked and Interleaved noTMS Trials}

We collected data in the absence of TMS either presented interleaved with TMS trials or as a separate block. It has previously been shown that infrequent interleaved noTMS trials can have oddball-like properties simply by being in a context where TMS pulses are administered during the majority of trials (Duecker \& Sack, 2013). This can cause unusual slowing of reaction times and, in the context of the present study, would invalidate their use as baseline condition. In line this view, a repeatedmeasures ANOVA on reaction time during noTMS trials with task (detection, angle judgment), TMS type (sham, real), and context (interleaved, blocked) as within subject factors revealed a highly significant effect of context $\left(F_{(1,13)}=59.940\right.$, $p<.001)$ and an effect of task $\left(F_{(1,13)}=379.706, p<.001\right)$ due to differences in task difficulty. The main effect of context resulted from faster reaction times during blocked noTMS trials compared to interleaved noTMS trials (mean difference $=22 \mathrm{~ms}$ ). This indicates that the mere presence of TMS trials creates a context that also modulates performance on interleaved trials without TMS. Consequently, we used blocked noTMS trials as baseline for subsequent analyses instead.

\section{Non-Neural Effect of TMS on Accuracy}

A repeated-measures ANOVA on accuracy with task (detection, clock), TMS type (real, sham), and TMS time window (-400 ms, -300 ms, .., +400 ms) as within subject factors revealed a significant three-way interaction $\left(F_{(8,104)}=3.867\right.$, $p=.008)$. Somewhat surprisingly, this already shows that non-neural effects of single TMS pulses depend on a complex interplay between various experimental factors, including nature of the experimental task and pulse timing, and that they are not equivalent for sham and real TMS. This interaction was further explored by analyzing the data for each task separately. For the detection task, a repeatedmeasures ANOVA on accuracy with TMS type and TMS time window as 
Detection Task

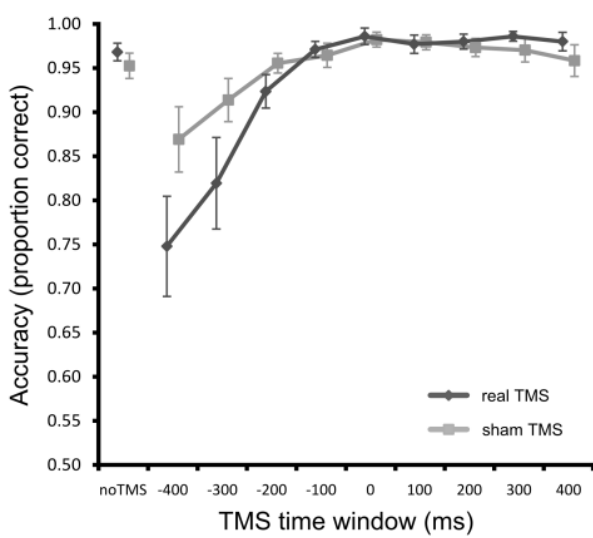

Angle Task

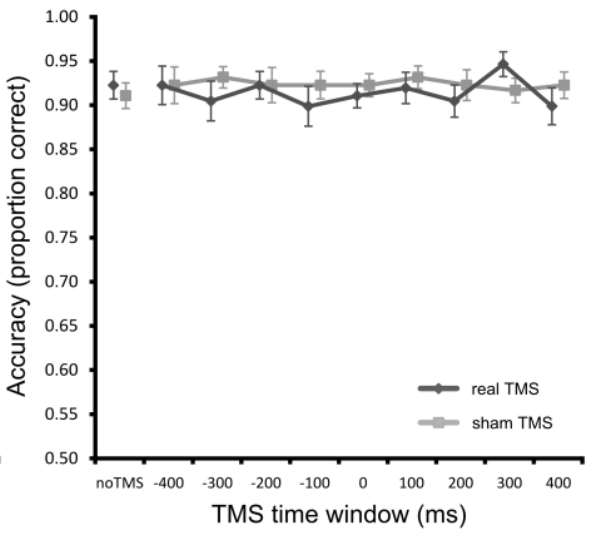

Figure 2. The effect of real and sham TMS on accuracy. For the detection task, decreased accuracy was observed at pre-stimulus TMS time windows with effects being stronger for real TMS compared to sham TMS. For the angle task, no effect of TMS was found on accuracy. Data points are slightly shifted sideways for clarity. Error bars show the standard error of the mean.

within subject factors revealed a significant main effect of TMS time window $\left(F_{(8,104)}=12.002, p=.001\right)$, no effect of TMS type $\left(F_{(1,13)}=3.37, p=.090\right)$, and a significant interaction $\left(F_{(8,104)}=5.478, p=.008\right)$. The main effect of TMS time window resulted from decreased accuracy (due to false alarms) during the earliest TMS time windows, when the TMS pulse preceded the target stimulus, which then gradually increased and leveled off when the TMS pulse temporally coincided with target appearance. However, as indicated by the interaction between TMS type and TMS time window, this impairment in task performance differed between real TMS and sham TMS (Figure 2). Post-hoc paired-samples t-tests (uncorrected) showed that the effect on accuracy was more pronounced when applying real TMS compared to sham TMS. This effect was strongest at $400 \mathrm{~ms}$ prior to target appearance $\left(t_{(13)}=2.986, p=.011\right)$ but a trend was still visible at $-300 \mathrm{~ms}\left(t_{(13)}=1.978\right.$, $p=.069)$ and-200 $\mathrm{ms}\left(t_{(13)}=1.759, p=.102\right)$. No significant differences were observed for all remaining TMS time windows (all $p>.10$ ).

For the angle judgment task, a repeated-measures ANOVA on accuracy with TMS type and TMS time window as within subject factors did not reveal any significant effects. Neither TMS type $\left(F_{(1,13)}=.863, p=.370\right)$ nor TMS time 
Detection Task

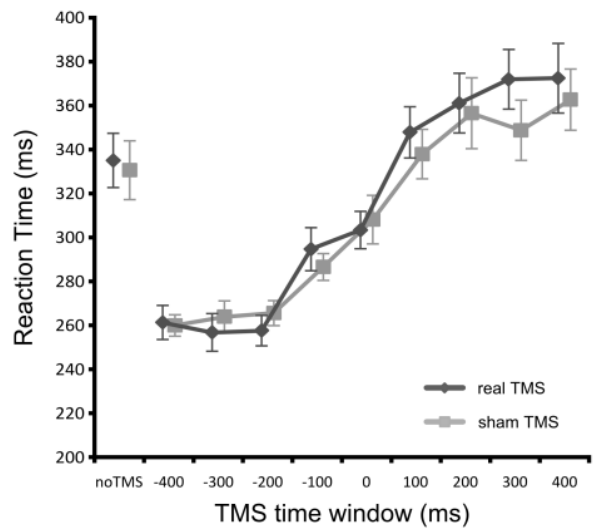

Angle Task

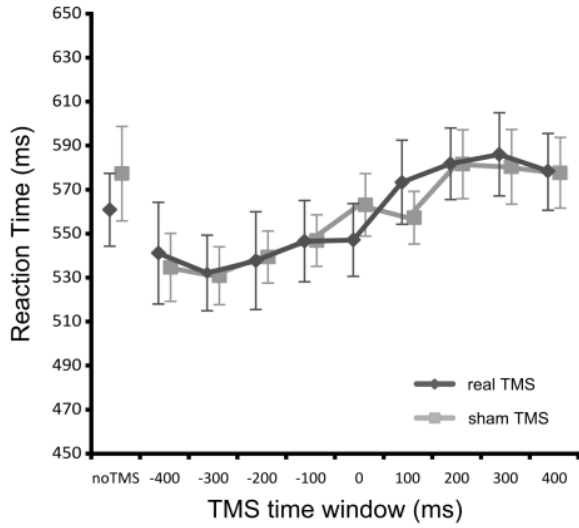

Figure 3. The effect of real and sham TMS on reaction times. For both tasks, reaction times were fastest at pre-stimulus TMS time windows and gradually increased with modulations still visible at post-stimulus TMS time windows. There was no difference between real and sham TMS. Data points are slightly shifted sideways for clarity. Error bars show the standard error of the mean.

window $\left(F_{(8,104)}=.548, p=.753\right)$ had an effect on accuracy and also the interaction was far from being significant $\left(F_{(8,104)}=.785, p=.617\right)$.

In summary, we found that TMS only had a non-neural effect on accuracy on the detection task whereas the angle judgment task was unaffected. Moreover, target detection was specifically impaired at pre-stimulus time windows with effects being stronger when applying real TMS compared to sham TMS. These effects were due to increased false alarm rates, reflecting failure to withhold an already prepared response when the TMS pulse was given.

\section{Non-Neural Effect of TMS on Reaction Time}

A repeated-measures ANOVA on reaction time with task (detection, clock), TMS type (real, sham), and TMS time window (-400 ms, -300 ms, ., , +400 ms) as within subject factors revealed significant main effects of task $\left(F_{(1,13)}=520.489, p<.001\right)$ and TMS time window $\left(F_{(8,104)}=47.010, p<.001\right)$. Moreover, we found a highly significant interaction between task and TMS time window $\left(F_{(8,104)}=20.740\right.$, $p<.001)$. The factor TMS type did not influence reaction time, neither the main 
effect nor any interaction with this factor reached significance (all $p>.10$ ). See Figure 3 for a complete overview of the data.

The main effect of task resulted from faster reaction times on the detection task compared to the clock task. This was simply due to differences in task difficulty because the detection task only required a button press after target appearance whereas the clock task involved angle judgments and two response alternatives.

The main effect of TMS time window resulted from gradually increasing reaction times across TMS time windows. Performance was best when the TMS pulse preceded target appearance and got worse the later the TMS pulse was applied with modulations still being visible for post-stimulus TMS time windows. However, as indicated by the interaction between task and TMS time window, this timedependence of task performance was not the same for both tasks. Consequently, we directly compared both tasks to examine this differential effect of TMS time window after subtracting the individual baseline for each participant (in order to remove global task effects). Additionally, we collapsed the data across the factor TMS type because this factor did not influence task performance. As above, this analysis revealed a highly significant interaction between task and TMS time window $\left(F_{(8,104)}\right.$ $=20.703, p<.001)$ with TMS effects being more pronounced at pre-stimulus time windows in the detection task compared to the angle judgment task (Figure 4). Paired-samples $t$-tests (uncorrected) statistically confirmed this effect, showing significant differences between tasks for early pre-stimulus TMS time windows at $400 \mathrm{~ms}\left(t_{(13)}=3.399, p=.005\right),-300 \mathrm{~ms}\left(t_{(13)}=2.975, p=.011\right)$, and $-200 \mathrm{~ms}$ $\left(t_{(13)}=2.863, p=.013\right)$. No significant differences were observed for all remaining TMS time windows (all $p>.15$ ) but there was a marginally significant effect at $+400 \mathrm{~ms}\left(t_{(13)}=2.057, p=.060\right)$.

In summary, we found that sham TMS and real TMS had the same effect on reaction times. Performance was modulated in a time-dependent way characterized by improvements at pre-stimulus TMS time windows and impairments at poststimulus TMS time windows. Moreover, the detection task was more vulnerable to these modulations than the angle judgment task which showed the same pattern but to a lesser extent. 


\section{Discussion}

It is often assumed that the non-neural side effects of TMS such as the clicking sound of the TMS pulse are non-specific, that is, they do not influence task performance dependent on TMS parameters. However, we have recently challenged this assumption on empirical grounds (Duecker \& Sack, 2013). Given that a valid interpretation of TMS results heavily relies on the adequacy of control conditions, and the fact that there are a range of controls to choose from (e.g. De Graaf \& Sack, 2011), we believe that it is essential to gather empirical knowledge of the non-neural effects of TMS. Here, we further developed the empirical basis of control strategies in TMS research. Specifically, we addressed the time-dependence and taskdependence of the non-neural effects of TMS and compared real and sham TMS over vertex.

The most important finding of the present study is that the non-neural effects of TMS depend on a complex interplay between pulse timing, experimental task, and real or sham TMS. For both outcome measures, we found remarkably specific effects of these factors that interacted in meaningful ways. In the absence of appropriate control conditions, such effects could easily be falsely attributed to the neural consequences of TMS. Our results caution against over-simplistic assumptions regarding the non-neural effects of TMS but also help designing wellcontrolled TMS experiments.

\section{Time-Dependence of Non-Neural Effects of TMS}

Previous work has shown that the clicking sound of a TMS pulse can facilitate target detection when applied prior to or in close temporal proximity of a target stimulus, primarily by acting as a warning signal and due to inter-sensory facilitation, respectively (Duecker \& Sack, 2013; Hackley, 2009; Marzi et al., 1998; Niemi \& Näätänen, 1981; Nikouline et al., 1999; Sawaki et al., 1999; Terao et al., 1997). Here, we investigated the non-neural effects of TMS for a broad range of TMS time windows including pre- and post-stimulus stimulation. To begin with, our results replicate earlier findings of pre-stimulus facilitation. A TMS pulse that is 
administered prior to a stimulus provides temporal information and, consequently, increases the readiness to respond resulting in decreased reaction times. Additionally, we found impaired task performance at post-stimulus time windows. Reaction times were considerably prolonged when the TMS pulse occurred late during a trial; probably due to expectancy violations that caused participants to 'wait' for the TMS pulse (as suggested, but not systematically investigated, by (De Graaf et al., 2009)).

Reaction times were not the only outcome measure that revealed a timedependent modulation of task performance. For the early pre-stimulus TMS time windows, we also found decreased accuracy on the detection task due to higher rates of false alarms. Apparently, participants were sometimes prone to press the response key when a TMS pulse was administered even though the task required them to only react when the target stimulus appeared. Such a failure to withhold an already prepared response until the proper 'go'-signal is presented is well known from the response inhibition literature (for a review, see [18]). As expected, this non-neural effect of TMS on accuracy was specific to the detection task because the clock task included two response alternatives so that response preparation could only occur once the stimulus was shown.

Taken together, this shows that there are time-dependent non-neural effects of TMS on reaction time and accuracy. Reaction times during pre- and post-stimulus TMS time windows were differentially affected by the clicking sound of the TMS coil leading to facilitation and impairment of task performance, respectively. This pattern of results was observed for the detection task as well as the clock task. In contrast, accuracies were only affected during pre-stimulus TMS time windows when target detection had to be reported by a speeded response with only one response alternative. A similar observation has recently been reported by Jacobs et al. (Jacobs, De Graaf, Goebel, \& Sack, 2012) in the context of behavioral priming and masking. When applying TMS over the vertex as a control condition, they found time-dependent modulations of prime effectiveness with stronger effects at prestimulus TMS time windows. Interestingly, these effects were specific to the priming task whereas no time-dependent effects were observed on masking. Moreover, these differential effects of TMS were only found with TMS over the 
vertex but absent during sham TMS over early visual cortex. These findings therefore also have a bearing on the task-dependence of the non-neural effects of TMS and the differences between real and sham TMS which are discussed below.

Importantly, these results demonstrate that a comparison between different TMS time windows can fail to control for the non-neural effects of TMS in the context of a TMS experiment. As a consequence, we recommend using control strategies that provide control conditions within each TMS time window and to avoid comparisons across time windows of stimulation.

\section{Task-Dependence of Non-Neural Effects of TMS}

One could argue that task performance on a simple detection task is particularly prone to be affected by the non-neural effects of TMS leaving the question in how far such results can be generalized. Other tasks that involve higher cognitive processes and require a more complex stimulus response mapping might be immune to such effects. Our results clearly show that the time-dependent effects on reaction time described above are qualitatively similar for the detection task and clock task even though the clock task consisted of a complex set of stimuli and required participants to (1) make an angle judgment (2) apply a decision rule based on this judgment, and (3) select the correct response alternative. It therefore seems highly plausible that similar non-neural effects of TMS can be observed across a broad range of tasks. Admittedly, both tasks used in the present study were reaction time tasks and accuracy on the clock task was unaffected by TMS. But, at the very least, our results show that the non-neural effects of TMS cannot be explained away as a low-level phenomenon with minimal scope.

While the presence of non-neural effects of TMS during both tasks is highly relevant for the generalizability of our findings, the differences between tasks are equally important. Interestingly, especially pre-stimulus effects on reaction times were more pronounced during the detection task compared to the clock task. Moreover, changes in accuracy were only observed for pre-stimulus TMS time windows during the detection task whereas the clock task was unaffected by TMS across all time windows. These differences between tasks have direct implications 
for the use of a control task as a control strategy in TMS experiments. A control task is one of the many possible approaches to demonstrate that a TMS effect is indeed 'neural'. The idea is that if stimulation of a brain area only affects performance on task ' $\mathrm{A}$ ' but not task ' $\mathrm{B}$ ' then the brain area in question is only functionally relevant for that particular task (De Graaf \& Sack, 2011). However, in case the non-neural effects of TMS also have a differential effect on the two tasks this reasoning obviously fails. Our results show that this can indeed be the case and a control task therefore has to be well-matched. Otherwise differences in the non-neural effects of TMS between tasks can be misinterpreted and produce false positive or false negative findings.

\section{Real versus Sham TMS}

We also compared the effect of real and sham TMS over the vertex. For the most part, we did not find significant differences between these two conditions with all effects on reaction time being identical. However, the drop in accuracy for prestimulus TMS time windows during the detection task was more pronounced when applying real TMS compared to sham TMS. While a neural explanation of this effect cannot be excluded, we propose that this increased rate of false alarms was due to somato-sensory effects. In the present study, a sham TMS coil was used that produced the same clicking sound as real TMS but almost completely lacked the somato-sensory effects that are typically associated with a TMS pulse. In our view, the combined auditory and somato-sensory effects act as a stronger trigger to press the response key than the clicking sound of the TMS coil in isolation. Our results therefore demonstrate that common sham TMS coils are an approximation of the non-neural effects of real TMS but are not perfect. They fail to mimic some nonneural aspects of TMS and, consequently, cannot control for them. Importantly, the somato-sensory effects during vertex stimulation are mild whereas other stimulation sites can cause stronger sensations and even discomfort. Under such circumstances, the differences between sham and real TMS become larger and most likely more problematic. For example, it has been found that subjective ratings of unpleasantness of TMS were negatively correlated with performance on a working memory task 
(Abler et al., 2005). However, the development of more advanced sham TMS coils is certainly possible and our results do not invalidate the use of existing sham TMS coils.

A widely used control strategy is to apply TMS over the vertex. It is assumed that this stimulation site has no neural effects and therefore qualifies as a baseline and control condition. As pointed out above, there were almost no differences between sham and real TMS except for the slightly stronger drop in accuracy at prestimulus TMS time windows during the detection task. On the one hand, these results indicate that real TMS over the vertex has advantages over sham TMS as a control condition because it accounts for mild somato-sensory effects of TMS without producing unintended neural effects on task performance. On the other hand, this advantage comes at the cost of reduced flexibility in TMS coil positioning. One of the strongest arguments in favor of sham TMS is that it can be applied to any brain area and is therefore better suited to control for stimulation sitedependent non-neural effects of TMS. For example, we have previously shown that a lateralized sham TMS pulls covert spatial attention towards the corresponding side of space thereby facilitating target detection in this hemifield (Duecker \& Sack, 2013). Such effects can hardly be controlled for with vertex stimulation. This illustrates that there is no perfect control strategy yet and, as a consequence, it is necessary to evaluate the pros and cons of each approach in the context of the specific research question at hand.

In conclusion, we provide novel evidence regarding the time-dependence and taskdependence of the non-neural effects of TMS and assessed the differences between real and sham TMS over vertex. Critically, we show that non-neural TMS effects depend on a complex interplay of these factors. Knowledge of such effects is of critical importance for the interpretation of TMS experiments and helps deciding what constitutes an appropriate control condition. Our results broaden the empirical basis of control strategies in TMS research and point at potential pitfalls that should be avoided. Otherwise, the non-neural effects of TMS can be falsely attributed to the intended TMS manipulation. 


\section{Acknowledgements}

This work was supported by the European Research Council under the European Union's Seventh Framework Programme (FP7/2007-2013) / ERC Grant agreement (263472 to A.T.S.), and the Netherlands Organization for Scientific Research (40007-230 to A.T.S \& E.F.). We thank our medical supervisor Cees van Leeuwen and independent physician Martin van Boxtel. 


\section{References}

Abler, B., Walter, H., Wunderlich, A., Grothe, J., Schönfeldt-Lecuona, C., Spitzer, M., \& Herwig, U. (2005). Side Effects of Transcranial Magnetic Stimulation Biased Task Performance in a Cognitive Neuroscience Study. Brain Topography, 17(4), 193-196.

Chambers, C. D., Garavan, H., \& Bellgrove, M. A. (2009). Neuroscience and Biobehavioral Reviews Insights into the neural basis of response inhibition from cognitive and clinical neuroscience. Neuroscience and Biobehavioral Reviews, 33, 631-646.

De Graaf, T. A., Jacobs, C., Roebroeck, A., \& Sack, A. T. (2009). FMRI effective connectivity and TMS chronometry: complementary accounts of causality in the visuospatial judgment network. PloS one, 4(12), e8307.

De Graaf, T. A., Roebroeck, A., Goebel, R., \& Sack, A. T. (2010). Brain Network Dynamics Underlying Visuospatial Judgment: An fMRI Connectivity Study. Journal of Cognitive Neuroscience, 22(9), 2012-2026.

De Graaf, T. A., \& Sack, A. T. (2011). Null results in TMS: from absence of evidence to evidence of absence. Neuroscience and Biobehavioral Reviews, 35(3), $871-877$.

Duecker, F., \& Sack, A. T. (2013). Pre-Stimulus Sham TMS Facilitates Target Detection. PLoS ONE, 8(3), e57765.

Hackley, S. A. (2009). The speeding of voluntary reaction by a warning signal. Psychophysiology, 46, 225-233.

Hallett, M. (2000). Transcranial magnetic stimulation and the human brain. Nature, 406(6792), 147-150. 
Jacobs, C., De Graaf, T. a, Goebel, R., \& Sack, A. T. (2012). The temporal dynamics of early visual cortex involvement in behavioral priming. PloS ONE, 7(11), e48808.

Jasper, H. H. (1958). The ten-twenty electrode system of the International Federation. Electroencephalography and Clinical Neurophysiology, 10, 371-375.

Marzi, C. A., Miniussi, C., Maravita, A., Bertolasi, L., Zanette, G., Rothwell, J. C., \& Sanes, J. N. (1998). Transcranial magnetic stimulation selectively impairs interhemispheric transfer of visuo-motor information in humans. Experimental Brain Research, 118(3), 435-8.

Niemi, P., \& Näätänen, R. (1981). Foreperiod and Simple Reaction Time. Psychological Bulletin, 89(1), 133-162.

Nikouline, V., Ruohonen, J., \& Ilmoniemi, R. J. (1999). The role of the coil click in TMS assessed with simultaneous EEG. Clinical Neurophysiology, 110(8), 1325-8.

Pascual-Leone, A., Walsh, V., \& Rothwell, J. (2000). Transcranial magnetic stimulation in cognitive neuroscience - virtual lesion, chronometry, and functional connectivity. Current Opinion in Neurobiology, 10(2), 232-237.

Sack, A. T. (2006). Transcranial magnetic stimulation, causal structure-function mapping and networks of functional relevance. Current Opinion in Neurobiology, 16(5), 593-599.

Sack, A. T., Kohler, A., Bestmann, S., Linden, D. E. J., Dechent, P., Goebel, R., \& Baudewig, J. (2007). Imaging the Brain Activity Changes Underlying Impaired Visuospatial Judgments: Simultaneous fMRI, TMS, and Behavioral Studies. Cerebral Cortex, 17(12), 2841-2852.

Sack, A. T., Sperling, J. M., Prvulovic, D., Formisano, E., Goebel, R., Di Salle, F., Dierks, T., et al. (2002). Tracking the Mind's Image in the Brain II : Transcranial Magnetic Stimulation Reveals Parietal Asymmetry in Visuospatial Imagery. Neron, $35,195-204$. 
Sawaki, L., Okita, T., Fujiwara, M., \& Mizuno, K. (1999). Specific and non-specific effects of transcranial magnetic stimulation on simple and go/no-go reaction time. Experimental Brain Research, 127(4), 402-8.

Terao, Y., Ugawa, Y., Suzuki, M., Sakai, K., Hanajima, R., Gemba-Shimizu, K., \& Kanazawa, I. (1997). Shortening of simple reaction time by peripheral electrical and submotor-threshold magnetic cortical stimulation. Experimental Brain Research, 115(3), 541-5.

Walsh, V., \& Cowey, A. (2000). Transcranial magnetic stimulation and cognitive neuroscience. Nature Reviews Neuroscience, 1(1), 73-79. 



\section{Chapter 7}

Summary and Conclusions 
The work described in this thesis is subdivided into two parts. In the first part, we used transcranial magnetic stimulation (TMS) to investigate hemispheric asymmetries in fronto-parietal networks underlying attentional control. In the second part, we focused on methodological aspects of TMS that are of particular importance in attention research but also have a bearing on TMS methodologies in general. In the following, we summarize the main findings of this thesis and discuss the implications of our work.

\section{Hemispheric Asymmetries in Fronto-Parietal Networks}

The simple fact that spatial neglect is more common and severe after damage to the right hemisphere has led to a long-standing debate in cognitive neuroscience regarding the mechanisms underlying attentional control (Corbetta \& Shulman, 2011; Heilman \& Abell, 1980; Kinsbourne, 1977; Mesulam, 1981). Today, this discussion is as lively as ever and there are three competing theories offering very different explanatory approaches. A detailed overview of these theories is provided in the general introduction of this thesis. Most importantly, they all predict hemispheric asymmetries in either dorsal or ventral fronto-parietal networks. While neuroimaging studies deserve all the credit for identifying these networks and their general functions (Corbetta \& Shulman, 2002), it has been proven difficult to assess the precise functional roles of the brain areas constituting these networks (Corbetta \& Shulman, 2011). In particular, hemispheric asymmetries have been rarely reported making it difficult to decide between competing theories of attentional control. In the last decade, TMS has provided a new perspective on hemispheric asymmetries especially in posterior parietal brain regions. The vast majority of studies report evidence in favor of Kinsbourne's “opponent processor” model (Kinsbourne, 1977) with impaired task performance commonly found only in the visual field contralateral to the hemisphere that was disrupted by TMS (Dambeck et al., 2006; Hilgetag, Theoret, \& Pascual-Leone, 2001; Silvanto, Muggleton, Lavie, \& Walsh, 2009). Moreover, there are indications for inter-hemispheric competition, e.g., with bilateral TMS having no effect instead of the combined effects one could expect based on unilateral stimulation (Dambeck et al., 2006). However, frontal brain areas 
have received far less attention and the existing work only confirmed their general involvement in attentional control but failed to reveal hemispheric asymmetries specific to shifts of spatial attention (Grosbras \& Paus, 2002; Muggleton, Juan, Cowey, \& Walsh, 2003; Silvanto, Lavie, \& Walsh, 2006; Smith, Jackson, \& Rorden, 2005).

In Chapter 2, we show for the first time that right and left frontal eye field (FEF) have functional properties that are in agreement with Heilman's "hemispatial" theory (Heilman \& Abell, 1980). Using a "virtual lesion" approach, we demonstrate that the right FEF mediates attention shifts to both hemifields whereas the left FEF only mediates attention shifts to the right hemifield. It therefore appears that the two models of attentional control as proposed by Kinsbourne and Heilman are not mutually exclusive. Instead, Kinsbourne's “opponent processor" model seems to apply to parietal cortex whereas Heilman's "hemispatial" theory seems to apply to frontal cortex. This finding is of direct relevance for current functional-anatomical models of attentional control.

In Chapter 3, we set out to extend our work over frontal cortex to posterior regions of the dorsal and ventral fronto-parietal network. Applying the same experimental design that has shown to be successful in our previous study, we aimed to investigate whether the above mentioned functional difference between frontal and parietal cortex indeed reflect general hemispheric asymmetries within the dorsal network or can be explained by other experimental factors such as task or TMS parameters. Moreover, we also applied TMS over the ventral network in order to examine its right-lateralization and interactions with the dorsal network as recently put forward by Corbetta and Shulman (Corbetta \& Shulman, 2011). Unfortunately, a suboptimal TMS coil positioning approach based on Talairach coordinates and high within-subject variability severely compromised the experiment leading to a null result. Despite this disappointing outcome, the research questions addressed here remain highly relevant and we are currently planning follow-up studies.

Taken together, the main finding of these content-driven chapters is that Heilman's "hemispatial" theory should not be discarded despite the strong evidence in favor of Kinsbourne's "opponent processor" model in parietal cortex. The existing evidence clearly suggests that different hemispheric asymmetries coexist 
within the dorsal network. Potentially the most important implication of this finding is that understanding spatial neglect is more complex that one might have initially thought. With Kinsbourne's “opponent processor” model receiving so much support, the last years have seen a strong interest to apply TMS not only as a research tool but also as a possible way to promote recovery in neglect patients after stroke (Alonso-Alonso, Fregni, \& Pascual-Leone, 2007; Bashir, Mizrahi, Weaver, Fregni, \& Pascual-Leone, 2010; Koch et al., 2012; Miniussi et al., 2008). Based on the principle of inter-hemispheric competition, the potential of non-invasive brain stimulation techniques has been explored by either directly "boosting" the lesioned hemisphere or by suppressing the contralesional hemisphere thereby reducing its inhibitory effect creating room for recovery. Obviously, the latter approach only makes sense in the context of Kinsbourne's "opponent processor" model. Thus far, clinical TMS studies have only applied this rationale to parietal cortex, matching the empirically discovered hemispheric asymmetries. Our results indicate that a simple transfer of these principles to frontal cortex might be ineffective because the underlying functional organization is different from parietal cortex. As a consequence, the present findings contribute to creating an evidence base for proper TMS interventions that are tailored to the specific functional network and brain area.

\section{Methodological Aspects of TMS research}

The content-driven experiments described in the first part of this thesis have also inspired work on methodological aspects of TMS research. An important prerequisite for investigating the behavioral consequences of TMS-induced brain activity changes is accurate TMS coil positioning. Only then, it is possible to reveal structure-functional relationships and to observe any TMS effect in the first place. The importance of accurate TMS coil positioning has been demonstrated empirically (Sack et al., 2009) with effect sizes being highest when using individual functional localization based on neuroimaging data. We used this approach for identifying TMS target points in chapter 2 and successfully interfered with attentional processes. In chapter 3 , however, we had to rely on a suboptimal approach based on individual anatomical data in combination with Talairach coordinates from the 
literature. In our view, this was a main reason for why we did not observe any TMS effect. There are many situations where individual functional data cannot be obtained and, for that reason, it is crucial to make best use of that data available. In Chapter 4, we presented a novel method for TMS coil position that only requires anatomical data for each participant but outperforms alternative approaches such as targeting based on Talairach coordinates. This so-called CBA-based approach relies on advanced whole-brain alignment schemes that exploit curvature information of the cortical surface in order to remove macro-anatomical variability across subjects (Fischl, Sereno, \& Dale, 1999; Frost \& Goebel, 2012). We show empirically that the CBA-based approach significantly improves localization of functional brain areas compared to traditional Talairach-based targeting. Given the widespread availability of cortex-based alignment schemes, the proposed procedure is easy to implement and at no additional measurement costs. Thus, our CBA-based approach for TMS coil positioning should be the method of choice either when individual functional data cannot be obtained or experimental factors argue against it (e.g. training or surprise effects).

In the final two chapters of this thesis, we explored the non-neural side effects of TMS. The clicking sound of the TMS coil and sensations on the head that accompany every TMS pulse create a strong need for appropriate control conditions in order to make sure that effects of interest are indeed the result of the intended brain activity changes. Surprisingly, there is hardly any empirical knowledge regarding the non-neural effects of TMS in the context of a given task performance. It is often assumed that these effects are unspecific, that is, they do not depend on TMS parameters such as stimulation site or time point of stimulation. In this thesis, we have challenged this assumption on empirical grounds. In Chapter 5, we showed that the clicking sound of a sham TMS coil can systematically influence task performance when applied prior to target appearance. Specifically, we observed facilitation of reaction times dependent on the TMS time window and TMS coil position due to a warning signal effect and exogenously triggered shifts of spatial attention, respectively. In Chapter 6, we showed that these facilitatory effects of prestimulus sham TMS generalize to a more complex task that involves higher cognitive functions and requires a more complex stimulus response mapping. 
Moreover, we found non-neural effects during post-stimulus TMS time windows with reaction times being slowed done because participants seem to 'wait' for the TMS pulse. Finally, we observed a non-neural effect on accuracies that depended not only on TMS time window and task but was stronger when applying real TMS over vertex compared with sham TMS. This difference clearly shows that both auditory and somato-sensory effects can have an influence on task performance with sham TMS only mimicking the former but not the latter side effect of TMS. This complex interplay of several factors emphasizes that the non-neural effects of TMS need to be carefully considered when designing an experiment and interpreting the data. Our results create an empirical basis of control strategies in TMS research and point at potential pitfalls that should be avoided. Importantly, based on our results, we advise to follow one general rule that should result in a well-controlled TMS experiment: TMS control conditions should always be orthogonal to the TMS factor of interest! To give an example, when using TMS chronometrically in order to reveal when a brain area is relevant for task performance, it is insufficient to directly compare different TMS time windows because differences between them might simply result from the non-neural effects of TMS. Instead, a separate control condition is required that also applies TMS during all time windows. Since we have shown task performance depends not only on the time point of stimulation but also the position of the TMS coil, the experimental task, and the presence of both auditory and somato-sensory effects, it is strongly advised to apply this reasoning to all common control strategies in TMS research. Finally, there is an experimental dilemma because it might not always be possible to control for all these possible confounders. Specifically, current sham TMS coils fail to mimic the sensations on the head when a TMS pulse is administered whereas any control conditions that makes use of real TMS necessarily has to be applied to another brain area so that the exact TMS coil position is no longer identical across TMS conditions. In the end, these factors have to be carefully considered in the light of the specific research question and experimental paradigm. Nevertheless, the current work not only increases awareness of these issues but is a first steps towards the empirical foundations of control strategies in TMS research. 


\section{References}

Alonso-Alonso, M., Fregni, F., \& Pascual-Leone, A. (2007). Brain stimulation in poststroke rehabilitation. Cerebrovascular Diseases, 24(suppl 1), 157-166.

Bashir, S., Mizrahi, I., Weaver, K., Fregni, F., \& Pascual-Leone, A. (2010). Assessment and modulation of neural plasticity in rehabilitation with transcranial magnetic stimulation. $P M \& R, 2(12$ Suppl 2), S253-S268.

Corbetta, M., \& Shulman, G. L. (2002). Control of goal-directed and stimulusdriven attention in the brain. Nature Reviews Neuroscience, 3(3), 201-215.

Corbetta, M., \& Shulman, G. L. (2011). Spatial neglect and attention networks. Annual Review of Neuroscience, 34(1), 569-599.

Dambeck, N., Sparing, R., Meister, I. G., Wienemann, M., Weidemann, J., Topper, R., \& Boroojerdi, B. (2006). Interhemispheric imbalance during visuospatial attention investigated by unilateral and bilateral TMS over human parietal cortices. Brain Research, 1072(1), 194-199.

Fischl, B., Sereno, M. I., \& Dale, A. M. (1999). Cortical Surface-Based Analysis II: Infaltion, Flattening, and a Surface-Based Coordinate System. NeuroImage, 9(2), 195-207.

Frost, M. A., \& Goebel, R. (2012). Measuring structural-functional correspondence: spatial variability of specialised brain regions after macro-anatomical alignment. NeuroImage, 59(2), 1369-1381. 5

Grosbras, M.-H., \& Paus, T. (2002). Transcranial magnetic stimulation of the human frontal eye field: effects on visual perception and attention. Journal of Cognitive Neuroscience, 14(7), 1109-1120.

Heilman, K. M., \& Abell, T. Van Den. (1980). Right hemisphere dominance for attention: The mechanism underlying hemispheric asymmetries of inattention (neglect). Neurology, 30(3), 327-330. 
Hilgetag, C. C., Theoret, H., \& Pascual-Leone, A. (2001). Enhanced visual spatial attention ipsilateral to rTMS-induced "virtual lesions" of human parietal cortex. Nature Neuroscience, 4(9), 953-957.

Kinsbourne, M. (1977). Hemi-neglect and hemisphere rivalry. Advances in Neurology, 18, 41-49.

Koch, G., Bonnì, S., Giacobbe, V., Bucchi, G., Basile, B., Lupo, F., Versace, V., et al. (2012). Theta-burst stimulation of the left hemisphere accelerates recovery of hemispatial neglect. Neurology, 78(1), 24-30.

Mesulam, M. M. (1981). A cortical network for directed attention and unilateral neglect. Annals of Neurology, 10(4), 309-325.

Miniussi, C., Cappa, S. F., Cohen, L. G., Floel, A., Fregni, F., Nitsche, M. a, Oliveri, M., et al. (2008). Efficacy of repetitive transcranial magnetic stimulation/transcranial direct current stimulation in cognitive neurorehabilitation. Brain Stimulation, 1(4), $326-336$.

Muggleton, N. G., Juan, C.-H., Cowey, A., \& Walsh, V. (2003). Human frontal eye fields and visual search. Journal of Neurophysiology, 89(6), 3340-3343.

Sack, A. T., Cohen Kadosh, R., Schuhmann, T., Moerel, M., Walsh, V., \& Goebel, R. (2009). Optimizing functional accuracy of TMS in cognitive studies: a comparison of methods. Journal of Cognitive Neuroscience, 21(2), 207-221.

Silvanto, J., Lavie, N., \& Walsh, V. (2006). Stimulation of the human frontal eye fields modulates sensitivity of extrastriate visual cortex. Journal of Neurophysiology, 96(2), 941-945.

Silvanto, J., Muggleton, N., Lavie, N., \& Walsh, V. (2009). The Perceptual and Functional Consequences of Parietal Top-Down Modulation on the Visual Cortex. Cerebral Cortex, 19(2), 327-330. 
Smith, D. T., Jackson, S. R., \& Rorden, C. (2005). Transcranial magnetic stimulation of the left human frontal eye fields eliminates the cost of invalid endogenous cues. Neuropsychologia, 43(9), 1288-1296. 



\section{Acknowledgements}

Writing has always been a painful exercise for me. I often feel deeply disappointed when my written words does not convey what I intend to express. Over the years, things have improved and it has been quite a while since I destroyed my laptop because of an approaching deadline that forced me to write too many words with too little time (that is, a couple of pages within a week). When it comes to scientific writing this dissertation hopefully shows that practice makes... at least decent. This acknowledgement section, however, brought back almost forgotten distress. How can I possibly get my thoughts on this piece of paper? My initial plan was to back down and keep it short and formal. But in the end I realized that I have no other choice but trying... I will keep it very short though.

Alex, you once said that the greatest threat to my PhD was that I might end up being fed up with everything and just quit.

Everybody, you made me stay! Science is great and everything, but I would have never ever finished my $\mathrm{PhD}$ without such colleagues. You made me feel welcome and accepted me for who I am. Some of you turned into close friends; there is nothing more I could have wished for.

Gesa, you are inseparably linked with this long journey that started almost ten years ago when I came to Maastricht. You are simply great!

Thank you all. 



\section{Curriculum Vitae}

Felix Dücker was born in Cologne, Germany on March $20^{\text {th }} 1981$. He graduated with the secondary school certificate in 2000 at the Humboldt Gymnasium in Cologne. In 2004, he enrolled at the Faculty of Psychology and Neuroscience at Maastricht University. During the final year of his undergraduate studies he participated in an exchange program and spent a semester in Victoria, Canada. He obtained his Bachelor of Science, cum laude, in Biological Psychology in 2006 and subsequently followed the Research Master program in Cognitive Neuroscience at Maastricht University. In his Master thesis, under supervision of Professor Alexander Sack, he conducted empirical work investigating effects of non-invasive brain stimulation on visual perception. He obtained his Master of Science, cum laude, in Cognitive Neuroscience in 2008. He then started the $\mathrm{PhD}$ program, under the supervision of Professor Alexander Sack and Professor Elia Formisano, at the Department of Cognitive Neuroscience at the Faculty of Psychology and Neuroscience. He is currently a post-doctoral fellow at the same institute where he continues his research. 



\section{Articles}

Duecker, F., Formisano, E., Sack, A. T. (2013). Hemispheric Differences in the Voluntary Control of Spatial Attention: Direct Evidence for a Right-Hemispheric Dominance within Frontal Cortex. Journal of Cognitive Neuroscience, 25(8), 13321342.

Duecker, F., \& Sack, A. T. (2013). Pre-Stimulus Sham TMS Facilitates Target Detection. PLoS ONE, 8(3): e57765.

Duecker, F., de Graaf, T. A., Jacobs, C., Sack, A. T. (2013). Time- and TaskDependent Non-Neural Effects of Real and Sham TMS. PLoS ONE, 8(0): e73813.

Platz, T., Roschka, S., Christel, M.I., Duecker, F., Rothwell, J.C., Sack, A.T. (2012). Early stages of motor skill learning and the specific relevance of the cortical motor system--a combined behavioural training and theta burst TMS study. Restorative Neurology and Neuroscience, 30, 199-211.

Duecker, F., Frost, M. A., de Graaf, T. A., Graewe, B., Jacobs, C., Goebel, R., Sack, A. T. (under review). The Cortex-Based Alignment Approach to TMS Coil Positioning.

\section{Conference Publications}

Duecker, F., Frost, M. A., de Graaf, T. A., Jacobs, C., Goebel, R., \& Sack, A. T. (2012). Improving TMS coil positioning by using macro-anatomical alignment to a probabilistic functional atlas. Poster presented at the annual meeting of Society for Neuroscience 2012, New Orleans, USA.

Goffaux, V., Duecker, F., Schiltz, C., \& Goebel, R. (2012). Orientation tuning for faces in the Fusiform Face Area and Primary Visual Cortex. Abstract presented at the annual meeting of Vision Sciences Society 2012. 
Duecker, F., \& Sack, A. T. (2012). Sham TMS Facilitates Target Detection. Poster presented at the Magstim Neuroscience Conference \& Workshop 2012, Oxford, England.

Duecker, F., Formisano, E., \& Sack, A. T. (2011). The functional asymmetry of left and right frontal eye field during voluntary shifts of spatial attention: direct evidence for right-hemispheric dominance. Poster presented at the annual meeting of Society for Neuroscience 2011, Washington DC, USA. 